JOURNAL OF THE

AMERICAN MATHEMATICAL SOCIETY

Volume 12, Number 2, April 1999, Pages 445-495

S 0894-0347(99)00290-8

\title{
WELL-POSEDNESS IN SOBOLEV SPACES OF THE FULL WATER WAVE PROBLEM IN 3-D
}

\author{
SIJUE WU
}

\section{$\S 1$. InTRODUCTION}

We consider the motion of the interface separating an inviscid, incompressible, irrotational fluid from a region of zero density in three-dimensional space; we assume that the fluid region is below the vacuum, the fluid is under the influence of gravity and the surface tension is zero. Assume that the density of mass of the fluid is one, the gravitational field is $(0,0,-1)$, the free interface is $\Sigma(t)$ at time $t \geq 0$, and the fluid occupies the region $\Omega(t)$. The motion of the fluid is described by

$$
\begin{array}{lcc}
v_{t}+v \cdot \nabla v=-(0,0,1)-\nabla p & \text { on } \Omega(t), t \geq 0 \quad \text { (Euler's equation), } \\
\operatorname{div} v=0 \quad \text { on } \Omega(t), t \geq 0 & \text { (incompressible), } \\
\operatorname{curl} v=0, \quad \text { on } \Omega(t), t \geq 0 & \text { (irrotational), }
\end{array}
$$

where $v=\left(v_{1}, v_{2}, v_{3}\right)$ is the fluid velocity, $p$ is the fluid pressure. Since we neglect the surface tension, the pressure is zero on the interface. So on the interface:

$$
p=0, \quad \text { on } \Sigma(t),
$$

$(1, v)$ is tangent to the free surface $(t, \Sigma(t))$.

We want to find solutions of system (1.1)-(1.5), taking prescribed initial data, such that for every fixed $t \geq 0, \Sigma(t)$ approaches the $x y$-plane at infinity, and $|v(x, y, z ; t)| \rightarrow 0,\left|v_{t}(x, y, z ; t)\right| \rightarrow 0$, as $|(x, y, z)| \rightarrow \infty$. Since the fluid is assumed irrotational, incompressible, we can reduce the study of the entire motion to the motion of the free surface.

The above model is a 3-D water wave model. It is generally known that when surface tension is neglected, the motion of the interface between an inviscid fluid and vacuum under the influence of gravity can be subject to Taylor instability [8], [22]. In a previous work [24], we studied the 2-dimensional water wave model; we showed that for a 2-D water wave, the sign condition relating to Taylor instability always holds for nonself-intersecting interface, that is, the motion of the interface is not subject to Taylor instability. We showed further that the 2-D full nonlinear water wave problem is uniquely solvable in Sobolev spaces, locally in time, for any initially nonself-intersecting interface. Earlier works on the well-posedness in Sobolev spaces of the 2-D water wave problem include Nalimov [18], Yosihara [25], and Walter Craig [6], where the main results concern the well-posedness of the

Received by the editors December 15, 1997 and, in revised form, August 24, 1998.

1991 Mathematics Subject Classification. Primary 76B15; Secondary 35L99, 35R35.

1991 Financial support provided in part by NSF grant DMS-9600141 and the J. Seward Johnson Sr. Charitable Trust. 
motion of free surface when it is a small perturbation of the still water. Shinbrot [19] and Kano and Nishida [12] obtained the local existence and uniqueness in time for both the 2-D and 3-D water wave problems with analytic data. T. Beale, T. Hou and Lowengrub [1] formulated the Taylor sign condition for an arbitrary solution and showed that when surface tension is neglected, the linearization of the 2-D full water wave equation about a presumed solution is well-posed provided the exact solution satisfies the Taylor sign condition. Recently, T. Hou, Z. Teng and P. Zhang [11] formulated the 3-D Taylor sign condition and extended the results in [1] to three-dimensional space. However a result on the well-posedness in Sobolev spaces of the full nonlinear 3-D water wave problem is still missing.

In this paper, we establish the well-posedness in Sobolev spaces of the full nonlinear 3-D water wave problem. We show that the results we obtained for the 2-D water wave also hold for $3-\mathrm{D}$, that is, the motion of the $3-\mathrm{D}$ water wave is not subject to Taylor instability and the full 3 -D water wave problem is well-posed in Sobolev spaces for any nonself-intersecting initial interface. The main difficulty is that in three-dimensional space, there is no such equivalence as the Riemann mapping, which we used in 2-D to "flatten out" the water wave and carry out the calculations; complex analysis does not apply to 3-D either. In this paper, we will show that Clifford analysis is an effective tool for the 3-D water wave problem, and we do not need Riemann Mapping to carry out the calculation and obtain well-posedness results.

The 3-D Taylor sign condition [11] is the following. Let $\xi=(x(\alpha, \beta, t), y(\alpha, \beta, t)$, $z(\alpha, \beta, t))$, where $-\infty<\alpha, \beta<\infty$, be the parameterization of the interface $\Sigma(t)$ by Lagrangian coordinates $(\alpha, \beta)$, that is, $\xi_{t}(\alpha, \beta, t)=v(\xi(\alpha, \beta, t), t)$ is the velocity and $\xi_{t t}(\alpha, \beta, t)$ is the acceleration of the particle occupying the position $\xi(\alpha, \beta, t)$ at time $t$. Let $\mathbf{n}$ be the unit normal vector of the interface $\Sigma(t)$ pointing out of the water region. The sign condition requires that the quantity

$$
\mathfrak{a}=\xi_{t t} \cdot \mathbf{n}-(0,0,-1) \cdot \mathbf{n} \geq c_{0}>0
$$

for some positive constant $c_{0}$ at each point on the interface. In order to understand our result on 2-D Taylor sign condition, Russ Caflisch and Tom Hou suggested the following physically more insightful approach. They pointed out that the quantity

$$
\mathfrak{a}=\xi_{t t} \cdot \mathbf{n}-(0,0,-1) \cdot \mathbf{n}=-\nabla p \cdot \mathbf{n},
$$

therefore $\mathfrak{a}>0$ is somewhat equivalent to the fact that $p>0$ inside the water region, and such a fact can be shown as long as the maximum principle applies. We find that indeed, the pressure $p$ is superharmonic in the water region $\Omega(t)$. We therefore are able to show that for the $3-\mathrm{D}$ water wave, the quantity $\mathfrak{a}$ is also pointwisely greater than a positive constant depending only on the geometry of the interface, as long as the interface is nonself-intersecting. A detailed proof will be given in $\S 4$. This proof also applies to $2-\mathrm{D}$, it is physically more transparent than our original proof in [24]. The rest of the paper is devoted to showing that this fact implies well-posedness of the full nonlinear 3-D water wave problem. Clifford analysis is our main analysis tool. Using Clifford algebra, we can rewrite the system (1.1)(1.5) into a system on the free surface, which can be easily reduced to a quasilinear system. The advantage of our new approach is that it works for all dimensions $n \geq 2$. This approach is however largely inspired by our previous work [24]. 
In the next section, we give a brief introduction to Clifford analysis; in $\S 3$, we introduce notations used in this paper and develop some identities; in $\S 4$, we formulate the system (1.1)-(1.5) into an equivalent system on the free surface and give a detailed proof for the fact that condition (1) always holds for nonself-intersecting free surfaces of $3-\mathrm{D}$ water waves; in $\S 5$, we reduce the full water wave system into a quasilinear system and, at the end of this section, we specify the initial data of the original water wave system, and convert it into initial data for the quasilinear system; in $\S 6$, we show that the quasilinear system is well-posed in certain classes of Sobolev spaces; and in $\S 7$, we prove that the solution of the quasilinear system satisfies the original nonlinear water wave equations, provided the initial data satisfy the compatibility condition given at the end of $\S 5$. We then conclude the well-posedness result for the full nonlinear 3-D water wave problem.

\section{$\S 2$. Clifford analysis}

In this section, we review a few basic notions and facts about Clifford analysis and potential theory that will be used in this paper. We refer the reader to [2], [10] and [15] for more on the subject of Clifford analysis.

Clifford numbers. Let $V_{n}$ be an $n$-dimensional real linear vector space with basis

$$
\left\{e_{1}, e_{2}, \ldots, e_{n}\right\}
$$

A Clifford algebra $\mathcal{C}\left(V_{n}\right)$ is a unitary, associative algebra over the reals, generated by $V_{n}$, under the product rule:

$$
e_{j}^{2}=-1, \quad j=1,2, \ldots, n ; \quad e_{i} e_{j}=-e_{j} e_{i}, \quad i \neq j .
$$

An element $\sigma \in \mathcal{C}\left(V_{n}\right)$ has a representation of the form

$$
\sigma=\sum \sigma_{I} e_{I}, \quad \sigma_{I} \in R
$$

where the summation is over all ordered $j$-tuples $I=\left\{h_{1}, \ldots, h_{j}\right\}, 0 \leq j \leq n, 1 \leq$ $h_{1}<\cdots<h_{j} \leq n$, and $e_{I}=e_{h_{1}} \ldots e_{h_{j}}$, with convention $e_{\emptyset}=1 ;\left\{\sigma_{I}\right\}$ are called components of $\sigma$; in particular, $\sigma_{\emptyset}$ is called the real part of $\sigma$ and is denoted by $\Re \sigma$. The length of $\sigma$ is $|\sigma|=\left(\sum \sigma_{I}^{2}\right)^{1 / 2} \cdot \mathcal{C}\left(V_{2}\right)$ is generally known as the algebra of quaternions. In the following, when we write $\sigma=\sum \sigma_{I} e_{I}$ or $\sigma=\sum \sigma_{i} e_{i}$, we always assume that $\sigma_{I}$ or $\sigma_{i}$ are real numbers. An element $\sigma=\sum \sigma_{I} e_{I} \in \mathcal{C}\left(V_{n}\right)$ is called a $p$-vector if the summation is over ordered $p$-tuples $I$ only. The conjugate of a 1-vector $\xi=\sum_{1}^{n} \xi_{i} e_{i}$ is defined as $\bar{\xi}=\sum_{1}^{n} \xi_{i} \bar{e}_{i}$, where $\bar{e}_{i}=-e_{i}, i=1, \ldots, n$. Given 1-vectors $\xi=\sum_{1}^{n} \xi_{i} e_{i}$ and $\eta=\sum_{1}^{n} \eta_{i} e_{i}$, their product $\xi \eta$ is the sum of the negative of their 'inner product' and 'outer product':

$$
\xi \eta=-\xi \cdot \eta+\xi \wedge \eta
$$

where $\xi \cdot \eta=\sum_{1}^{n} \xi_{i} \eta_{i}$ and $\xi \wedge \eta=\frac{1}{2}(\xi \eta-\eta \xi)$. The inner product $\xi \cdot \eta$ is a scalar, and the outer product $\xi \wedge \eta$ is a 2 -vector. Notice that when $n=3$, the components of $\xi \wedge \eta$ are the same as that of the cross product $\xi \times \eta$, when $\xi$ and $\eta$ are regarded as vectors in 3 -space. We will identify the notations $\xi \wedge \eta$ and $\xi \times \eta$ in the following sections when we study $3-\mathrm{D}$ water waves. 
Clifford analyticity. We begin with some notations. Let $f$ be a function defined on $R^{d}$. We denote by $\partial^{k} f$ the $k$-th order partial derivative $\partial_{x_{1}}^{k_{1}} \ldots \partial_{x_{d}}^{k_{d}} f$, where $k=k_{1}+\cdots+k_{d}$. We say $f \in C^{j}\left(R^{d}\right)$ if $f$ is $j$-times continuously differentiable, and all the $k$-th order derivatives $\partial^{k} f, k=0,1, \ldots, j$, satisfy $\sup _{x \in R^{d}}\left|\partial^{k} f(x)\right|<\infty$; if the function $f$ is $\mathcal{C}\left(V_{n}\right)$-valued, we write $f \in C^{j}\left(R^{d}, \mathcal{C}\left(V_{n}\right)\right)$ or $f \in C^{j}\left(R^{d}\right)$. If $f$ is defined and continuous on a manifold $M$, we write $f \in C(M)$; we write $f \in C\left(M, \mathcal{C}\left(V_{n}\right)\right)$ or $f \in C(M)$ if $f$ is $\mathcal{C}\left(V_{n}\right)$-valued. For $M \subset R^{n}$, we say $f \in C_{0}(M)$ if $f \in C(M)$ and $f$ is compactly supported.

Let $\Omega \subset R^{n}$ be a domain. We denote by $\bar{\Omega}$ the closure of $\Omega$, and by $\Omega^{c}$ the complement of $\Omega$. Assume $\Sigma$ is the boundary of $\Omega$. We say $\Omega$ is a Lipschitz domain with Lipschitz constant less than or equal to $M$, if

1. for each $\xi \in \Sigma$, there is a ball or a half-space $E \ni \xi$, a coordinate system $x^{\prime}=\left(x_{1}, \ldots, x_{n-1}\right), x_{n}$, with origin at $\xi$, and a function $\phi: R^{n-1} \rightarrow R$ such that $\phi(0)=0$,

$$
\left|\phi\left(x^{\prime}\right)-\phi\left(y^{\prime}\right)\right| \leq M\left|x^{\prime}-y^{\prime}\right|, \text { and } \Omega \cap E=\left\{\eta=\left(x^{\prime}, x_{n}\right): x_{n}>\phi\left(x^{\prime}\right)\right\} \cap E ;
$$

2. $\Sigma$ can be covered by finitely many $E$ 's from the above collection.

If for each $\xi \in \Sigma$, the function $\phi$ can be chosen in $C^{j}\left(R^{n-1}\right)$, we say $\Omega$ is a $C^{j}$ domain. For a $C^{j}$ domain $\Omega, j \geq 1$, we say $f \in C^{1}(\Sigma)$ if $f$ is 1-time continuously differentiable; we say $f \in C_{0}^{1}(\Sigma)$ if $f \in C^{1}(\Sigma)$ and $f$ is compactly supported. Let $B_{r}(0)$ be the ball centered at the origin with radius $r$. For an unbounded domain $\Omega$, we say $\Sigma$ approaches plane $x_{n}=0$ at infinity, if there is a sufficiently large $R$, such that outside $B_{R}(0), \Sigma$ is a graph $\left(x^{\prime}, \phi\left(x^{\prime}\right)\right)$ and $\lim _{\left|x^{\prime}\right| \rightarrow \infty} \phi\left(x^{\prime}\right)=0$.

There are a few different but equivalent ways to define Clifford analyticity. We use the one given in [10].

Let $W$ be a domain in $R^{n}, \mathcal{D}=\sum_{1}^{n} e_{i} \partial_{x_{i}}$, and $\overline{\mathcal{D}}=\sum_{1}^{n} \bar{e}_{i} \partial_{x_{i}}$. A function $f \in C\left(W, \mathcal{C}\left(V_{n}\right)\right)$ is said to be Clifford analytic on $W$ if $\mathcal{D} f=0$ everywhere on $W$. Since $\overline{\mathcal{D}} \mathcal{D}=\mathcal{D} \overline{\mathcal{D}}=\Delta_{n}$, where $\Delta_{n}$ is the Laplacian, the real components of a Clifford analytic function are necessarily harmonic; on the other hand, if $\phi$ is a harmonic function on $W$, then $\overline{\mathcal{D}} \phi$ and $\mathcal{D} \phi(=-\overline{\mathcal{D}} \phi)$ are Clifford analytic functions. For a 1 -vector-valued function $f=\sum_{1}^{n} f_{i} e_{i}$, we have $\mathcal{D} f=-\operatorname{div} f+\operatorname{curl} f$, so $f=\sum_{1}^{n} f_{i} e_{i}$ is Clifford analytic if and only if

$$
\operatorname{div} f=0 \quad \text { and } \quad \operatorname{curl} f=0 .
$$

It is clear that when $n=2$, Clifford analyticity for 1-vector-valued functions is equivalent to complex (anti)analyticity.

Let $\Gamma_{n}$ be the fundamental solution for $\Delta$ in $R^{n}$ :

$$
\Gamma_{2}(\xi)=\frac{1}{2 \pi} \log |\xi|, \quad \Gamma_{n}(\xi)=\frac{1}{\omega_{n}(2-n)}|\xi|^{-(n-2)}, \quad n>2,
$$

where $\omega_{n}$ is the surface area of the unit sphere in $R^{n}$, and let

$$
K(\xi)=2 \overline{\mathcal{D}} \Gamma(\xi)=\frac{2}{\omega_{n}} \frac{\bar{\xi}}{|\xi|^{n}}, \quad \xi \neq 0 .
$$

Assume that $\Omega$ is a bounded $C^{2}$ domain in $W$ with boundary $\Sigma$. For each $\xi \in \Sigma$, let $\mathbf{n}(\xi)=\sum_{1}^{n} n_{i} e_{i}$ be the unit outer normal to $\Sigma$, and let $d S$ be the surface measure of $\Sigma$. We have the following Cauchy Integral Theorem. 
Theorem 2.1 (see [10, Chapter 2], (3.20)Corollary). If $f$ is a Clifford analytic function on $W$, then

$$
f(\eta)=\frac{1}{\omega_{n}} \int_{\Sigma} \frac{\overline{\xi-\eta}}{|\xi-\eta|^{n}} \mathbf{n}(\xi) f(\xi) d S(\xi)
$$

for each $\eta$ in the interior of $\Omega$.

Let $f$ be a $\mathcal{C}\left(V_{n}\right)$-valued function on $\Sigma$. We introduce the Hilbert transform of $f$ on $\Sigma$ :

$$
H^{\Sigma} f(\xi)=\frac{2}{\omega_{n}} p \cdot v \cdot \int_{\Sigma} \frac{\overline{\xi^{\prime}-\xi}}{\left|\xi^{\prime}-\xi\right|^{n}} \mathbf{n}\left(\xi^{\prime}\right) f\left(\xi^{\prime}\right) d S\left(\xi^{\prime}\right), \quad \text { for } \xi \in \Sigma
$$

where p.v. $\int$ denotes the principal value of the integral.

Theorem 2.2 (see [10, Chapter 2], (7.1)Theorem). Assume that $f \in C^{1}\left(\Sigma, \mathcal{C}\left(V_{n}\right)\right)$. Then the Cauchy integral

$$
C f(\eta)=\frac{1}{\omega_{n}} \int_{\Sigma} \frac{\overline{\xi-\eta}}{|\xi-\eta|^{n}} \mathbf{n}(\xi) f(\xi) d S(\xi), \quad \eta \in \Omega
$$

is Clifford analytic on $\Omega$, and continues on $\bar{\Omega}$. Moreover

$$
C f(\xi)=\frac{1}{2} f(\xi)+\frac{1}{2} H^{\Sigma} f(\xi), \quad \text { for } \xi \in \Sigma .
$$

Theorem 2.3 (see [10, Chapter 2], (7.21)Corollary). Assume that $f \in C\left(\bar{\Omega}, \mathcal{C}\left(V_{n}\right)\right)$. $f$ is Clifford analytic on $\Omega$ if and only if

$$
f(\xi)=H^{\Sigma} f(\xi), \quad \text { for } \xi \in \Sigma .
$$

Remark 1. For an unbounded $C^{2}$ domain $\Omega$, Theorems 2.1, 2.2 and 2.3 hold under the further assumption that $f$ decay at infinity.

Remark 2. Assume further that $\Sigma$ approaches plane $x_{n}=0$ at infinity. Then $H^{\Sigma} 1=p . v \cdot \int_{\Sigma} K\left(\xi^{\prime}-\xi\right) \mathbf{n}\left(\xi^{\prime}\right) d S\left(\xi^{\prime}\right)=0$ for $\xi \in \Sigma$. This is an easy consequence of $(3.22)$ in $[10$, Chapter 2].

We introduce a few further notations and results. Assume $\Omega$ is a $C^{2}$ domain in $R^{n}$ with boundary $\Sigma$ and outer unit normal $\mathbf{n}$. The so-called double layered potential operator $\mathcal{K}$ is defined for the scalar-valued function $f$ on $\Sigma$ by

$$
\mathcal{K} f(\xi)=\frac{2}{\omega_{n}} p \cdot v \cdot \int_{\Sigma} \frac{\left(\xi^{\prime}-\xi\right) \cdot \mathbf{n}\left(\xi^{\prime}\right)}{\left|\xi^{\prime}-\xi\right|^{n}} f\left(\xi^{\prime}\right) d S\left(\xi^{\prime}\right), \quad \text { for } \xi \in \Sigma,
$$

and the single layered potential operator $\mathcal{S}$ is defined by

$$
\mathcal{S} f(\eta)=\frac{1}{\omega_{n}(2-n)} \int_{\Sigma} \frac{1}{|\eta-\xi|^{n-2}} f(\xi) d S(\xi), \quad \text { for } \eta \in R^{n} .
$$

We know from a straightforward calculation that

$$
\overline{\mathcal{D}} \mathcal{S} f=C(\mathbf{n} f) .
$$

Let $L^{2}(\Sigma, d S)$ be the $L^{2}$ space on $\Sigma$ with respect to surface measure $d S$. We denote by $\mathcal{K}^{*}$ the adjoint of $\mathcal{K}$ in $L^{2}(\Sigma, d S)$, that is,

$$
\mathcal{K}^{*} f(\xi)=-\frac{2}{\omega_{n}} p \cdot v \cdot \int_{\Sigma} \frac{\left(\xi^{\prime}-\xi\right) \cdot \mathbf{n}(\xi)}{\left|\xi^{\prime}-\xi\right|^{n}} f\left(\xi^{\prime}\right) d S\left(\xi^{\prime}\right), \quad \text { for } \xi \in \Sigma .
$$

The following result is due to Verchota [23]; see also [13], [14]. 
Theorem 2.4 ([23], [13], [14]). Assume that $\Omega$ and $\Omega^{c}$ are unbounded, connected Lipschitz domains, and $\Sigma$ approaches plane $x_{n}=0$ at infinity. Then $I \pm \mathcal{K}$ : $L^{2}(\Sigma, d S) \rightarrow L^{2}(\Sigma, d S)$ and their adjoints $I \pm \mathcal{K}^{*}: L^{2}(\Sigma, d S) \rightarrow L^{2}(\Sigma, d S)$ are invertible.

Remark. The invertibility result given by Verchota in [23] is for bounded Lipschitz domains, and by Kenig in [13, Theorem 2.1.5] is for unbounded domains where the boundary is a single Lipschitz graph. Notice that the key elements in their proof are the Rellich identity [23, Lemma 2.2], [13, Lemma 2.1.8] and a continuity method [13, Lemma 2.1.7]. For the type of domain in Theorem 2.4, Rellich identity [23, Lemma 2.2] holds under the assumption that $u \in C^{\infty}(\Omega), \Delta u=0$ in $\Omega$, and $u(\eta)=O\left(|\eta|^{2-n}\right), \nabla u(\eta)=O\left(|\eta|^{1-n}\right)$ at $\infty$. Notice that for any $f \in C_{0}(\Sigma)$, $u=\mathcal{S} f$ satisfies these assumptions on both $\Omega$ and $\Omega^{c}$. The proof in [23], [13] can be modified to give Theorem 2.4.

The following results are consequences of Theorem 2.4 and Coifman-McIntoshMeyer [4]. Let $\Omega$ be as in Theorem 2.4, and let $N(u)$ be a nontangential maximal function of $u$ on $\Omega$.

Theorem 2.5 ([23], [13], [14]). Let $\Omega$ be as in Theorem 2.4. There is a unique solution $u$ of the Dirichlet problem

$$
\begin{aligned}
& \Delta u=0 \quad \text { in } \Omega, \\
& u=f \in L^{2}(\Sigma, d S)
\end{aligned}
$$

such that $N(u) \in L^{2}(\Sigma, d S)$, where the boundary values are taken nontangentially a.e. Moreover, the solution $u$ has the form

$$
u(\eta)=\frac{2}{\omega_{n}} \int_{\Sigma} \frac{(\xi-\eta) \cdot \mathbf{n}(\xi)}{|\xi-\eta|^{n}}(I+\mathcal{K})^{-1} f(\xi) d S(\xi), \quad \eta \in \Omega .
$$

Remark. We can also solve the Dirichlet problem (D) by Kelvin transform (see [7, page 147]). That is, we first solve the Dirichlet problem on the bounded domain $\tilde{\Omega}$

$$
\begin{array}{lr}
\Delta \tilde{u}=0 & \text { in } \tilde{\Omega}, \\
\tilde{u}=\tilde{f} & \text { on } \partial \tilde{\Omega},
\end{array}
$$

where $\tilde{\Omega}=\left\{\eta \mid \frac{\eta}{|\eta|^{2}} \in \Omega\right\}$ (w.l.o.g. we assume $0 \notin \bar{\Omega}$ ) and $\tilde{f}(\xi)=|\xi|^{2-n} f\left(\frac{\xi}{|\xi|^{2}}\right)$. We then take

$$
u(\eta)=|\eta|^{2-n} \tilde{u}\left(\frac{\eta}{|\eta|^{2}}\right), \quad \eta \in \Omega
$$

The function $u$ in (2.16) is a solution of (D). Assume that $\Omega$ is a $C^{2}$ domain, and $\Sigma$ is given by the graph $\left(x^{\prime}, \phi\left(x^{\prime}\right)\right)$ outside a sufficiently large ball, with $\lim _{\left|x^{\prime}\right| \rightarrow \infty} \phi\left(x^{\prime}\right)=$ $0, \lim _{\left|x^{\prime}\right| \rightarrow \infty} \nabla \phi\left(x^{\prime}\right)$, and $\lim _{\left|x^{\prime}\right| \rightarrow \infty} \nabla \nabla \phi\left(x^{\prime}\right)$ exists and is finite. For $f \in C_{0}^{1}(\Sigma)$, a solution given by (2.16) clearly satisfies $u(\eta)=o\left(|\eta|^{2-n}\right)$ and $\nabla u(\eta)=o\left(|\eta|^{1-n}\right)$, as $|\eta| \rightarrow \infty$. It is easy to check that the solution $u$ in (2.16) is the same as the one in (2.15) if $f \in C_{0}(\Sigma)$, and more generally, if $f \in C(\Sigma)$ and $f$ decay as fast as $o\left(|\eta|^{2-n}\right)$ at infinity. 
Theorem 2.6 ([23], [13], [14]). Let $\Omega$ be as in Theorem 2.4. There is a unique solution $u$ of the Neumann problem

$$
\begin{aligned}
& \Delta u=0 \quad \text { in } \Omega, \\
& \frac{\partial u}{\partial \mathbf{n}}=f \in L^{2}(\Sigma, d S)
\end{aligned}
$$

such that $N(\nabla u) \in L^{2}(\Sigma, d S)$, where the boundary values are taken nontangentially a.e. Moreover, the solution $u$ has the form

$$
u(\eta)=\frac{2}{\omega_{n}(n-2)} \int_{\Sigma} \frac{1}{|\xi-\eta|^{n-2}}\left(-I+\mathcal{K}^{*}\right)^{-1} f(\xi) d S(\xi), \quad \eta \in \Omega .
$$

In the next section, we will introduce more notations and develop some identities.

\section{§3. General notations and basic identities}

We make further preparations in this section. Since we are only concerned with the 3 - $\mathrm{D}$ water wave, we present this section in the 3 -D context.

For the 3 -D water wave, we use Clifford algebra $\mathcal{C}\left(V_{3}\right)$. We regard points (vectors) $\xi=(x, y, z) \in R^{3}$ and their corresponding Clifford 1-vectors $\xi=x e_{1}+y e_{2}+z e_{3}$ as equivalent, the same notation $\xi$ can either be a point or its corresponding 1vector in different contexts: for example, for vectors $\xi$ and $\eta$, the multiplication $\xi \eta$ is obtained through Clifford multiplication by regarding $\xi, \eta$ as their Clifford 1-vector counterparts; for Clifford 1-vectors $\xi, \eta$ and $\zeta, \xi(\eta \times \zeta)$ is obtained by first regarding $\eta$ and $\zeta$ as vectors and calculating the cross product $\eta \times \zeta$, then rewriting $\eta \times \zeta$ as it's corresponding Clifford 1-vector and calculating the Clifford multiplication between $\xi$ and $\eta \times \zeta$. This also applies to 1-vectors of operators such as $\mathcal{D}=\partial_{x} e_{1}+\partial_{y} e_{2}+\partial_{z} e_{3}$, of which $\nabla=\left(\partial_{x}, \partial_{y}, \partial_{z}\right)$ is the vector counterpart.

We give some additional notations of functional spaces. We use $\mathfrak{S}\left(R^{d}\right)$ to indicate the Schwartz class of functions on $R^{d}$, i.e. those functions which are infinitely differentiable, and rapidly decay at infinity. $\mathfrak{S}^{\prime}\left(R^{d}\right)$ is the distribution space on $R^{d} . L_{p}\left(R^{d}\right)$ is the $L_{p}$ space on $R^{d}$ with norm $\|u\|_{L_{p}}=\left(\int|u(x)|^{p} d x\right)^{1 / p}$. We use $H^{s}\left(R^{2}\right),-\infty<s<\infty$, to indicate Sobolev space which consists of $u \in \mathfrak{S}^{\prime}\left(R^{2}\right)$ such that $(1+|\xi|)^{s} \hat{u}(\xi) \in L_{2}\left(R^{2}\right)$, and the Sobolev norm of $u \in H^{s}\left(R^{2}\right)$ is

$$
\|u\|_{s}=\left(\int(1+|\xi|)^{2 s}|\hat{u}(\xi)|^{2} d \xi\right)^{1 / 2} .
$$

$H^{s}\left(R^{2} ; \mathcal{C}\left(V_{3}\right)\right)$ is the space of $\mathcal{C}\left(V_{3}\right)$-valued $\mathfrak{u}$ such that each component $u_{I}$ of $\mathfrak{u}$ is in $H^{s}\left(R^{2}\right)$; the norm of $\mathfrak{u} \in H^{s}\left(R^{2} ; \mathcal{C}\left(V_{3}\right)\right)$ is given by $\|\mathfrak{u}\|_{s}=\left(\sum_{I}\left\|u_{I}\right\|_{s}^{2}\right)^{1 / 2}$. We put $\|u\|=\|u\|_{0}$ for $u \in H^{0}\left(R^{2}\right)=L_{2}\left(R^{2}\right)$ or $u \in H^{0}\left(R^{2}, \mathcal{C}\left(V_{3}\right)\right)$. For any function space $\mathcal{A}$, we say $u=u(\cdot, t) \in C^{j}([0, T], \mathcal{A})$ if the mapping $t \in[0, T] \rightarrow u(\cdot, t)$ is $j$-times continuously differentiable from $[0, T]$ to $\mathcal{A}$, and we write $u(t)=u(\cdot, t)$ for fixed $t$. For $f=f(\alpha, \beta, t)$, we use $\partial_{t} f$ or $f_{t}$ to denote the partial derivative w.r.t. $t$, $\partial_{\alpha} f$ or $f_{\alpha}$ to denote the derivative w.r.t. $\alpha$, and $\partial^{k} f$ to denote the $k$-th order partial derivative $\partial_{\alpha}^{k_{1}} \partial_{\beta}^{k_{2}} f$, where $k=k_{1}+k_{2}$. For operators $A, B,[A, B]=A B-B A$ is the commutator. A pseudo-differential operator $P(D)$ is defined by, for $u \in \mathfrak{S}\left(R^{2}\right)$,

$$
P(D) u=(2 \pi)^{-2} \iint P(\xi) \hat{u}(\xi) e^{i x \cdot \xi} d \xi .
$$

For each fixed $t \in[0, T]$, assume $\xi=\xi(\alpha, \beta, t)=x(\alpha, \beta, t) e_{1}+y(\alpha, \beta, t) e_{2}+$ $z(\alpha, \beta, t) e_{3},-\infty<\alpha, \beta<\infty$, describes a surface $\Sigma(t)$, which divides space $R^{3}$ into two unbounded, simply connected, lower and upper $C^{2}$ domains $\Omega(t)$ and $\Omega(t)^{c}$. 
Assume further that $\xi \in C^{1}\left([0, T], C^{2}\left(R^{2}, \mathcal{C}\left(V_{3}\right)\right)\right), \xi_{\alpha} \times \xi_{\beta} \neq 0$ and $\mathbf{n}=\frac{\xi_{\alpha} \times \xi_{\beta}}{\left|\xi_{\alpha} \times \xi_{\beta}\right|}$ gives the outer unit normal of $\Omega(t)$. We define the Hilbert transform on $\Sigma(t)$ of $f=f(\alpha, \beta, t)$ by

$$
H_{\Sigma(t)} f(\alpha, \beta, t)=H^{\Sigma(t)} F(\xi(\alpha, \beta, t), t),
$$

where $F=F(\xi, t)$ is the function on $\Sigma(t)$ such that $f(\alpha, \beta, t)=F(\xi(\alpha, \beta, t), t)$. That is,

$$
\begin{aligned}
H_{\Sigma(t)} f(\alpha, \beta, t) & =H^{\Sigma(t)} F(\xi(\alpha, \beta, t), t) \\
& =\frac{2}{\omega_{3}} p \cdot v \cdot \int_{\Sigma(t)} \frac{\overline{\xi^{\prime}-\xi(\alpha, \beta, t)}}{\left|\xi^{\prime}-\xi(\alpha, \beta, t)\right|^{3}} \mathbf{n}\left(\xi^{\prime}\right) F\left(\xi^{\prime}, t\right) d S\left(\xi^{\prime}\right) \\
& =p . v . \iint K\left(\xi\left(\alpha^{\prime}, \beta^{\prime}, t\right)-\xi(\alpha, \beta, t)\right)\left(\xi_{\alpha^{\prime}}^{\prime} \times \xi_{\beta^{\prime}}^{\prime}\right) f\left(\alpha^{\prime}, \beta^{\prime}, t\right) d \alpha^{\prime} d \beta^{\prime} .
\end{aligned}
$$

In a brief form, we write (3.1) as

$$
H_{\Sigma(t)} f=p . v . \iint K\left(\xi^{\prime}-\xi\right)\left(\xi_{\alpha^{\prime}}^{\prime} \times \xi_{\beta^{\prime}}^{\prime}\right) f^{\prime} d \alpha^{\prime} d \beta^{\prime},
$$

where $\xi, \xi^{\prime}, \xi_{\alpha^{\prime}}^{\prime}, \xi_{\beta^{\prime}}^{\prime}$ and $f^{\prime}$ stand for $\xi(\alpha, \beta, t), \xi\left(\alpha^{\prime}, \beta^{\prime}, t\right), \partial_{\alpha^{\prime}} \xi\left(\alpha^{\prime}, \beta^{\prime}, t\right), \partial_{\beta^{\prime}} \xi\left(\alpha^{\prime}, \beta^{\prime}, t\right)$ and $f\left(\alpha^{\prime}, \beta^{\prime}, t\right)$ respectively. Similarly, $\xi_{t}^{\prime}$ and $\xi_{t}$ will be used later as the brief forms of $\partial_{t} \xi\left(\alpha^{\prime}, \beta^{\prime}, t\right)$ and $\partial_{t} \xi(\alpha, \beta, t)$. We define the single, double layered potentials $\mathcal{S}$, $\mathcal{K}$ and the adjoint $\mathcal{K}^{*}$ on $f=f(\alpha, \beta, t)$ similarly as $\mathcal{S} f=\mathcal{S} F \circ \xi, \mathcal{K} f=\mathcal{K} F \circ \xi$ and $\mathcal{K}^{*} f=\mathcal{K}^{*} F \circ \xi$. We have the following identities.

Lemma 3.1. Let $f=f(\alpha, \beta, t) \in C\left([0, T], \mathfrak{S}\left(R^{2}\right)\right)$. Then

$$
\left[\partial_{t}, H_{\Sigma(t)}\right] f=p . v . \iint K\left(\xi^{\prime}-\xi\right)\left(\left(\xi_{t}-\xi_{t}^{\prime}\right) \times\left(\xi_{\beta^{\prime}}^{\prime} f_{\alpha^{\prime}}^{\prime}-\xi_{\alpha^{\prime}}^{\prime} f_{\beta^{\prime}}^{\prime}\right)\right) d \alpha^{\prime} d \beta^{\prime}
$$

and

$$
\begin{aligned}
& {\left[\partial_{\alpha}, H_{\Sigma(t)}\right] f=p . v . \iint K\left(\xi^{\prime}-\xi\right)\left(\left(\xi_{\alpha}-\xi_{\alpha^{\prime}}^{\prime}\right) \times\left(\xi_{\beta^{\prime}}^{\prime} f_{\alpha^{\prime}}^{\prime}-\xi_{\alpha^{\prime}}^{\prime} f_{\beta^{\prime}}^{\prime}\right)\right) d \alpha^{\prime} d \beta^{\prime}} \\
& {\left[\partial_{\beta}, H_{\Sigma(t)}\right] f=p . v . \iint K\left(\xi^{\prime}-\xi\right)\left(\left(\xi_{\beta}-\xi_{\beta^{\prime}}^{\prime}\right) \times\left(\xi_{\beta^{\prime}}^{\prime} f_{\alpha^{\prime}}^{\prime}-\xi_{\alpha^{\prime}}^{\prime} f_{\beta^{\prime}}^{\prime}\right)\right) d \alpha^{\prime} d \beta^{\prime} .}
\end{aligned}
$$

Proof. We prove (3.2) only. (3.3) can be obtained similarly.

We first calculate the quantity

$$
-(\eta \cdot \nabla) K\left(\xi_{\alpha^{\prime}}^{\prime} \times \xi_{\beta^{\prime}}^{\prime}\right)+\left(\xi_{\alpha^{\prime}}^{\prime} \cdot \nabla\right) K\left(\eta \times \xi_{\beta^{\prime}}^{\prime}\right)+\left(\xi_{\beta^{\prime}}^{\prime} \cdot \nabla\right) K\left(\xi_{\alpha^{\prime}}^{\prime} \times \eta\right),
$$

where $\eta$ is a (Clifford 1-)vector. Assume $K=K_{1} e_{1}+K_{2} e_{2}+K_{3} e_{3}$, where $K_{i}$, $i=1,2,3$, are components of $K$. For $i=1,2,3$, we have, in vector form, that

$$
\begin{aligned}
& -\left(\eta \cdot \nabla K_{i}\right) \xi_{\alpha^{\prime}}^{\prime} \times \xi_{\beta^{\prime}}^{\prime}+\left(\xi_{\alpha^{\prime}}^{\prime} \cdot \nabla K_{i}\right) \eta \times \xi_{\beta^{\prime}}^{\prime}+\left(\xi_{\beta^{\prime}}^{\prime} \cdot \nabla K_{i}\right) \xi_{\alpha^{\prime}}^{\prime} \times \eta \\
& =-\left(\eta \cdot \nabla K_{i}\right) \xi_{\alpha^{\prime}}^{\prime} \times \xi_{\beta^{\prime}}^{\prime}+\eta \times\left(\left(\xi_{\alpha^{\prime}}^{\prime} \cdot \nabla K_{i}\right) \xi_{\beta^{\prime}}^{\prime}-\left(\xi_{\beta^{\prime}}^{\prime} \cdot \nabla K_{i}\right) \xi_{\alpha^{\prime}}^{\prime}\right) \\
& =-\left(\eta \cdot \nabla K_{i}\right) \xi_{\alpha^{\prime}}^{\prime} \times \xi_{\beta^{\prime}}^{\prime}+\eta \times\left(\left(\xi_{\alpha^{\prime}}^{\prime} \times \xi_{\beta^{\prime}}^{\prime}\right) \times \nabla K_{i}\right) \\
& =-\left(\eta \cdot \nabla K_{i}\right) \xi_{\alpha^{\prime}}^{\prime} \times \xi_{\beta^{\prime}}^{\prime}+\left(\eta \cdot \nabla K_{i}\right) \xi_{\alpha^{\prime}}^{\prime} \times \xi_{\beta^{\prime}}^{\prime}-\left(\eta \cdot\left(\xi_{\alpha^{\prime}}^{\prime} \times \xi_{\beta^{\prime}}^{\prime}\right)\right) \nabla K_{i} \\
& =-\left(\eta \cdot\left(\xi_{\alpha^{\prime}}^{\prime} \times \xi_{\beta^{\prime}}^{\prime}\right)\right) \nabla K_{i} .
\end{aligned}
$$


The Clifford algebra version of (3.4) is

$$
\begin{aligned}
& -\left(\eta \cdot \nabla K_{i}\right) \xi_{\alpha^{\prime}}^{\prime} \times \xi_{\beta^{\prime}}^{\prime}+\left(\xi_{\alpha^{\prime}}^{\prime} \cdot \nabla K_{i}\right) \eta \times \xi_{\beta^{\prime}}^{\prime}+\left(\xi_{\beta^{\prime}}^{\prime} \cdot \nabla K_{i}\right) \xi_{\alpha^{\prime}}^{\prime} \times \eta \\
& \quad=-\left(\eta \cdot\left(\xi_{\alpha^{\prime}}^{\prime} \times \xi_{\beta^{\prime}}^{\prime}\right)\right) \mathcal{D} K_{i} .
\end{aligned}
$$

Since $K=\overline{\mathcal{D}} \Gamma$, where $\Gamma$ is the fundamental solution for Laplacian $\Delta$, we have $\sum_{1}^{3} e_{i} \mathcal{D} K_{i}(\xi)=0$ for $\xi \neq 0$. Therefore

$$
\begin{aligned}
&-(\eta \cdot \nabla) K(\xi)\left(\xi_{\alpha^{\prime}}^{\prime} \times \xi_{\beta^{\prime}}^{\prime}\right)+\left(\xi_{\alpha^{\prime}}^{\prime} \cdot \nabla\right) K(\xi)\left(\eta \times \xi_{\beta^{\prime}}^{\prime}\right)+\left(\xi_{\beta^{\prime}}^{\prime} \cdot \nabla\right) K(\xi)\left(\xi_{\alpha^{\prime}}^{\prime} \times \eta\right) \\
&=\sum_{1}^{3} e_{i}\left\{-\left(\eta \cdot \nabla K_{i}(\xi)\right)\left(\xi_{\alpha^{\prime}}^{\prime} \times \xi_{\beta^{\prime}}^{\prime}\right)\right.+\left(\xi_{\alpha^{\prime}}^{\prime} \cdot \nabla K_{i}(\xi)\right)\left(\eta \times \xi_{\beta^{\prime}}^{\prime}\right) \\
&\left.+\left(\xi_{\beta^{\prime}}^{\prime} \cdot \nabla K_{i}(\xi)\right)\left(\xi_{\alpha^{\prime}}^{\prime} \times \eta\right)\right\} \\
&=-\left(\eta \cdot\left(\xi_{\alpha^{\prime}}^{\prime} \times \xi_{\beta^{\prime}}^{\prime}\right)\right) \sum_{1}^{3} e_{i} \mathcal{D} K_{i}(\xi)=0, \quad \text { for } \xi \neq 0 .
\end{aligned}
$$

Now let's prove (3.2). By definition, we have

$$
\begin{aligned}
{\left[\partial_{t}, H_{\Sigma(t)}\right] f } & =\partial_{t}\left(H_{\Sigma(t)} f\right)-H_{\Sigma(t)}\left(\partial_{t} f\right) \\
& =p . v \cdot \iint \partial_{t}\left(K\left(\xi^{\prime}-\xi\right)\left(\xi_{\alpha^{\prime}}^{\prime} \times \xi_{\beta^{\prime}}^{\prime}\right)\right)\left(f^{\prime}-f(\alpha, \beta, t)\right) d \alpha^{\prime} d \beta^{\prime} \\
& =p . v \cdot \iint \partial_{t}\left(K\left(\xi^{\prime}-\xi\right)\right)\left(\xi_{\alpha^{\prime}}^{\prime} \times \xi_{\beta^{\prime}}^{\prime}\right)\left(f^{\prime}-f(\alpha, \beta, t)\right) d \alpha^{\prime} d \beta^{\prime} \\
& +p . v \cdot \iint K\left(\xi^{\prime}-\xi\right)\left(\xi_{t \alpha^{\prime}}^{\prime} \times \xi_{\beta^{\prime}}^{\prime}+\xi_{\alpha^{\prime}}^{\prime} \times \xi_{t \beta^{\prime}}^{\prime}\right)\left(f^{\prime}-f(\alpha, \beta, t)\right) d \alpha^{\prime} d \beta^{\prime} .
\end{aligned}
$$

Notice that

$$
\partial_{t}\left(K\left(\xi^{\prime}-\xi\right)\right)=\left(\left(\xi_{t}^{\prime}-\xi_{t}\right) \cdot \nabla\right) K\left(\xi^{\prime}-\xi\right), \quad \partial_{\alpha^{\prime}} K\left(\xi^{\prime}-\xi\right)=\left(\xi_{\alpha^{\prime}}^{\prime} \cdot \nabla\right) K\left(\xi^{\prime}-\xi\right)
$$

and

$$
\partial_{\beta^{\prime}} K\left(\xi^{\prime}-\xi\right)=\left(\xi_{\beta^{\prime}}^{\prime} \cdot \nabla\right) K\left(\xi^{\prime}-\xi\right)
$$

In (3.5) we take $\eta=\xi_{t}^{\prime}-\xi_{t}$ and apply to (3.6). We get

$$
\begin{aligned}
{\left[\partial_{t}, H_{\Sigma(t)}\right] f } & =p . v \cdot \iint\left\{\partial_{\alpha^{\prime}} K\left(\left(\xi_{t}^{\prime}-\xi_{t}\right) \times \xi_{\beta^{\prime}}^{\prime}\right)\right. \\
& \left.\quad+\partial_{\beta^{\prime}} K\left(\xi_{\alpha^{\prime}}^{\prime} \times\left(\xi_{t}^{\prime}-\xi_{t}\right)\right)\right\}\left(f^{\prime}-f\right) d \alpha^{\prime} d \beta^{\prime} \\
& + \text { p.v. } \iint K\left(\xi^{\prime}-\xi\right)\left(\xi_{t \alpha^{\prime}}^{\prime} \times \xi_{\beta^{\prime}}^{\prime}+\xi_{\alpha^{\prime}}^{\prime} \times \xi_{t \beta^{\prime}}^{\prime}\right)\left(f^{\prime}-f(\alpha, \beta, t)\right) d \alpha^{\prime} d \beta^{\prime} .
\end{aligned}
$$


Applying integration by parts to the first term on the right hand side of (3.7), we obtain

$$
\begin{aligned}
{\left[\partial_{t}, H_{\Sigma(t)}\right] f } & =-p . v \cdot \iint K\left(\xi^{\prime}-\xi\right)\left(\left(\xi_{t}^{\prime}-\xi_{t}\right) \times \xi_{\beta^{\prime}}^{\prime} f_{\alpha^{\prime}}^{\prime}+\xi_{\alpha^{\prime}}^{\prime} \times\left(\xi_{t}^{\prime}-\xi_{t}\right) f_{\beta^{\prime}}^{\prime}\right) d \alpha^{\prime} d \beta^{\prime} \\
& - \text { p.v. } \iint K\left(\xi^{\prime}-\xi\right)\left(\xi_{t \alpha^{\prime}}^{\prime} \times \xi_{\beta^{\prime}}^{\prime}+\xi_{\alpha^{\prime}}^{\prime} \times \xi_{t \beta^{\prime}}^{\prime}\right)\left(f^{\prime}-f(\alpha, \beta, t)\right) d \alpha^{\prime} d \beta^{\prime} \\
& + \text { p.v. } \iint K\left(\xi^{\prime}-\xi\right)\left(\xi_{t \alpha^{\prime}}^{\prime} \times \xi_{\beta^{\prime}}^{\prime}+\xi_{\alpha^{\prime}}^{\prime} \times \xi_{t \beta^{\prime}}^{\prime}\right)\left(f^{\prime}-f(\alpha, \beta, t)\right) d \alpha^{\prime} d \beta^{\prime} \\
& =\text { p.v. } \iint K\left(\xi^{\prime}-\xi\right)\left(\left(\xi_{t}-\xi_{t}^{\prime}\right) \times\left(\xi_{\beta^{\prime}}^{\prime} f_{\alpha^{\prime}}^{\prime}-\xi_{\alpha^{\prime}}^{\prime} f_{\beta^{\prime}}^{\prime}\right)\right) d \alpha^{\prime} d \beta^{\prime} .
\end{aligned}
$$

This gives (3.2).

Let $f=f(\alpha, \beta, t)$ be a scalar-valued function in $C\left([0, T], \mathfrak{S}\left(R^{2}\right)\right)$, and let $F(\xi(\alpha, \beta, t), t)=f(\alpha, \beta, t)$. Let $f^{\hbar}=f^{\hbar}(\eta, t), \eta \in \Omega(t)$, be the harmonic extension of $F=F(\xi, t)$ on $\Omega(t)$ given by Theorem 2.5. We denote by $\nabla_{\xi} f$ the restriction of $\nabla f^{\hbar}$ on $\Sigma(t)$; that is,

$$
\nabla_{\xi} f(\alpha, \beta, t)=\nabla f^{\hbar}(\xi(\alpha, \beta, t), t),
$$

and similarly $\mathcal{D}_{\xi} f(\alpha, \beta, t)=\mathcal{D} f^{\hbar}(\xi(\alpha, \beta, t), t), \partial_{x} f(\alpha, \beta, t)=\partial_{x} f^{\hbar}(\xi(\alpha, \beta, t), t)$, etc. We call $\nabla_{\xi} f$ the space variable gradient of $f$, to distinguish from the usual gradient $\nabla f=\left(f_{\alpha}, f_{\beta}\right)$. We define the normal derivative of $f$ by

$$
\nabla_{\mathbf{n}} f(\alpha, \beta, t)=\mathbf{n}(\xi(\alpha, \beta, t)) \cdot \nabla_{\xi} f(\alpha, \beta, t)=\frac{\partial f^{\hbar}}{\partial \mathbf{n}}(\xi(\alpha, \beta, t), t)
$$

and we denote by $\nabla_{T} f$ the quantity

$$
\nabla_{T} f(\alpha, \beta, t)=\mathbf{n}(\xi(\alpha, \beta, t)) \times \nabla_{\xi} f(\alpha, \beta, t) .
$$

$\nabla_{\mathbf{n}} f$ is generally known as the Dirichlet-Neumann operator. We get by definition that

$$
\begin{aligned}
\nabla_{\xi} f & =-\mathbf{n}(\xi) \mathbf{n}(\xi) \nabla_{\xi} f=-\mathbf{n}(\xi)\left(-\mathbf{n}(\xi) \cdot \nabla_{\xi} f+\mathbf{n}(\xi) \times \nabla_{\xi} f\right) \\
& =\mathbf{n}(\xi) \nabla_{\mathbf{n}} f-\mathbf{n}(\xi) \nabla_{T} f
\end{aligned}
$$

and

$$
\begin{aligned}
\nabla_{T} f & =\mathbf{n}(\xi) \times \nabla_{\xi} f=\frac{1}{\left|\xi_{\alpha} \times \xi_{\beta}\right|}\left(\xi_{\alpha} \times \xi_{\beta}\right) \times \nabla_{\xi} f \\
& =\frac{1}{\left|\xi_{\alpha} \times \xi_{\beta}\right|}\left(\xi_{\beta}\left(\xi_{\alpha} \cdot \nabla f^{\hbar}(\xi)\right)-\xi_{\alpha}\left(\xi_{\beta} \cdot \nabla f^{\hbar}(\xi)\right)\right. \\
& =\frac{1}{\left|\xi_{\alpha} \times \xi_{\beta}\right|}\left(\xi_{\beta} \partial_{\alpha} f-\xi_{\alpha} \partial_{\beta} f\right)=T_{1} \partial_{\alpha} f-T_{2} \partial_{\beta} f
\end{aligned}
$$

where $T_{1}=\frac{1}{\left|\xi_{\alpha} \times \xi_{\beta}\right|} \xi_{\beta}, T_{2}=\frac{1}{\left|\xi_{\alpha} \times \xi_{\beta}\right|} \xi_{\alpha}$.

Since $f^{\hbar}$ is harmonic on $\Omega, \mathcal{D} f^{\hbar}$ is Clifford analytic. So by Theorem 2.3, $\mathcal{D}_{\xi} f=$ $H_{\Sigma(t)} \mathcal{D}_{\xi} f$, consequently

$$
\mathbf{n}(\xi) \mathcal{D}_{\xi} f=\mathbf{n}(\xi) H_{\Sigma(t)} \mathcal{D}_{\xi} f=\mathbf{n}(\xi) p . v . \iint K\left(\xi^{\prime}-\xi\right)\left(\xi_{\alpha^{\prime}}^{\prime} \times \xi_{\beta^{\prime}}^{\prime}\right) \mathcal{D}_{\xi}^{\prime} f^{\prime} d \alpha^{\prime} d \beta^{\prime}
$$


and

$$
\begin{aligned}
\nabla_{\mathbf{n}} f & =-\Re\left(\mathbf{n}(\xi) \mathcal{D}_{\xi} f\right) \\
& =-p . v \cdot \iint \mathbf{n}(\xi) \cdot K\left(\xi^{\prime}-\xi\right)\left(\left(\xi_{\alpha^{\prime}}^{\prime} \times \xi_{\beta^{\prime}}^{\prime}\right) \cdot \nabla_{\xi}^{\prime} f^{\prime}\right) d \alpha^{\prime} d \beta^{\prime} \\
& +p . v \cdot \iint\left(\mathbf{n}(\xi) \times K\left(\xi^{\prime}-\xi\right)\right) \cdot\left(\left(\xi_{\alpha^{\prime}}^{\prime} \times \xi_{\beta^{\prime}}^{\prime}\right) \times \nabla_{\xi}^{\prime} f^{\prime}\right) d \alpha^{\prime} d \beta^{\prime} \\
& =-\mathcal{K}^{*}\left(\nabla_{\mathbf{n}} f\right)+p . v \cdot \iint\left(\mathbf{n}(\xi) \times K\left(\xi^{\prime}-\xi\right)\right) \cdot\left(\xi_{\beta^{\prime}}^{\prime} \partial_{\alpha^{\prime}} f^{\prime}-\xi_{\alpha^{\prime}}^{\prime} \partial_{\beta^{\prime}} f^{\prime}\right) d \alpha^{\prime} d \beta^{\prime}
\end{aligned}
$$

therefore

$$
\left(I+\mathcal{K}^{*}\right)\left(\nabla_{\mathbf{n}} f\right)=p . v \cdot \iint\left(\mathbf{n}(\xi) \times K\left(\xi^{\prime}-\xi\right)\right) \cdot\left(\xi_{\beta^{\prime}}^{\prime} \partial_{\alpha^{\prime}} f^{\prime}-\xi_{\alpha^{\prime}}^{\prime} \partial_{\beta^{\prime}} f^{\prime}\right) d \alpha^{\prime} d \beta^{\prime}
$$

On the other hand, we know from Theorem 2.6 that $f^{\hbar}=2 \mathcal{S}\left(-I+\mathcal{K}^{*}\right)^{-1} \nabla_{\mathbf{n}} f$, so from (2.13) and Theorem 2.2, $\mathcal{D} f^{\hbar}=-2 C\left(\mathbf{n}\left(-I+\mathcal{K}^{*}\right)^{-1} \nabla_{\mathbf{n}} f\right)$ is continuous on $\overline{\Omega(t)}$ and

$$
\mathcal{D}_{\xi} f=-\left(I+H_{\Sigma(t)}\right)\left(\mathbf{n}\left(-I+\mathcal{K}^{*}\right)^{-1} \nabla_{\mathbf{n}} f\right) .
$$

We introduce one more notation in this section. We define

$$
H_{\Sigma}^{*}=\mathbf{n} H_{\Sigma} \mathbf{n}
$$

A simple calculation shows that for a real scalar-valued function $f, \Re\left(H_{\Sigma}^{*} f\right)=\mathcal{K}^{*} f$.

We calculate the commutators $\left[\partial_{t}, \nabla_{\mathbf{n}}\right],\left[\partial_{\alpha}, \nabla_{\mathbf{n}}\right]$ and $\left[\partial_{\beta}, \nabla_{\mathbf{n}}\right]$ in the following lemma.

Lemma 3.2. For $f \in C\left([0, T], \mathfrak{S}\left(R^{2}\right)\right)$, we have

$$
\begin{aligned}
& \left(I+\mathcal{K}^{*}\right)\left(\left[\partial_{t}, \nabla_{\mathbf{n}}\right] f\right) \\
& =\Re\left(-\mathbf{n}_{t} H_{\Sigma(t)}\left(\mathcal{D}_{\xi} f\right)-\mathbf{n}\left[\partial_{t}, H_{\Sigma(t)}\right]\left(\mathcal{D}_{\xi} f\right)+\mathbf{n} H_{\Sigma(t)}\left(\mathbf{n}_{t} \mathbf{n} \mathcal{D}_{\xi} f\right)\right) \\
& +\int_{\Sigma(t)} \mathbf{n}(\xi) \times K\left(\xi^{\prime}-\xi\right) \cdot\left(\partial_{t}\left(\frac{1}{\left|\xi_{\alpha^{\prime}}^{\prime} \times \xi_{\beta^{\prime}}^{\prime}\right|} \xi_{\beta^{\prime}}\right) f_{\alpha^{\prime}}-\partial_{t}\left(\frac{1}{\left|\xi_{\alpha^{\prime}}^{\prime} \times \xi_{\beta^{\prime}}^{\prime}\right|} \xi_{\alpha^{\prime}}\right) f_{\beta^{\prime}}\right) d S\left(\xi^{\prime}\right)
\end{aligned}
$$

and

$$
\begin{aligned}
& \left(I+\mathcal{K}^{*}\right)\left(\left[\partial_{\alpha}, \nabla_{\mathbf{n}}\right] f\right) \\
& =\Re\left(-\mathbf{n}_{\alpha} H_{\Sigma(t)}\left(\mathcal{D}_{\xi} f\right)-\mathbf{n}\left[\partial_{\alpha}, H_{\Sigma(t)}\right]\left(\mathcal{D}_{\xi} f\right)+\mathbf{n} H_{\Sigma(t)}\left(\mathbf{n}_{\alpha} \mathbf{n} \mathcal{D}_{\xi} f\right)\right) \\
& +\int_{\Sigma(t)} \mathbf{n}(\xi) \times K\left(\xi^{\prime}-\xi\right) \cdot\left(\partial_{\alpha^{\prime}}\left(\frac{1}{\left|\xi_{\alpha^{\prime}}^{\prime} \times \xi_{\beta^{\prime}}^{\prime}\right|} \xi_{\beta^{\prime}}\right) f_{\alpha^{\prime}}-\partial_{\alpha^{\prime}}\left(\frac{1}{\left|\xi_{\alpha^{\prime}}^{\prime} \times \xi_{\beta^{\prime}}^{\prime}\right|} \xi_{\alpha^{\prime}}\right) f_{\beta^{\prime}}\right) d S\left(\xi^{\prime}\right),
\end{aligned}
$$

where $\mathbf{n}_{t}$ and $\mathbf{n}_{\alpha}$ are partial derivatives of $\mathbf{n}$ w.r.t. $t$ and $\alpha$ respectively. A similar identity also holds for $\left[\partial_{\beta}, \nabla_{\mathbf{n}}\right] f$.

Proof. We only derive (3.16). (3.17) can be proved similarly.

By definition, we have that

$$
\begin{aligned}
\mathbf{n} \mathcal{D}_{\xi} f & =\mathbf{n} H_{\Sigma(t)}\left(\mathcal{D}_{\xi} f\right)=-H_{\Sigma(t)}^{*}\left(\mathbf{n} \mathcal{D}_{\xi} f\right) \quad \text { and } \\
\mathbf{n} \mathcal{D}_{\xi} f_{t} & =\mathbf{n} H_{\Sigma(t)}\left(\mathcal{D}_{\xi} f_{t}\right)=-H_{\Sigma(t)}^{*}\left(\mathbf{n} \mathcal{D}_{\xi} f_{t}\right) .
\end{aligned}
$$


Therefore

$$
\begin{aligned}
\partial_{t}\left(\mathbf{n} \mathcal{D}_{\xi} f\right)-\mathbf{n} \mathcal{D}_{\xi} f_{t} & =-\partial_{t}\left(H_{\Sigma(t)}^{*}\left(\mathbf{n} \mathcal{D}_{\xi} f\right)\right)+H_{\Sigma(t)}^{*}\left(\mathbf{n} \mathcal{D}_{\xi} f_{t}\right) \\
& =-\left[\partial_{t}, H_{\Sigma(t)}^{*}\right]\left(\mathbf{n} \mathcal{D}_{\xi} f\right)-H_{\Sigma(t)}^{*}\left(\partial_{t}\left(\mathbf{n} \mathcal{D}_{\xi} f\right)-\mathbf{n} \mathcal{D}_{\xi} f_{t}\right)
\end{aligned}
$$

We know

$$
\begin{aligned}
& \partial_{t}\left(\mathbf{n} \mathcal{D}_{\xi} f\right)-\mathbf{n} \mathcal{D}_{\xi} f_{t} \\
& =-\left(\partial_{t}\left(\mathbf{n} \cdot \mathcal{D}_{\xi} f\right)-\mathbf{n} \cdot \mathcal{D}_{\xi} f_{t}\right)+\partial_{t}\left(\mathbf{n} \times \mathcal{D}_{\xi} f\right)-\mathbf{n} \times \mathcal{D}_{\xi} f_{t} \\
& =-\left(\partial_{t}\left(\nabla_{\mathbf{n}} f\right)-\left(\nabla_{\mathbf{n}} f_{t}\right)\right) \\
& +\partial_{t}\left(\frac{1}{\left|\xi_{\alpha} \times \xi_{\beta}\right|}\left(\xi_{\beta} f_{\alpha}-\xi_{\alpha} f_{\beta}\right)\right)-\frac{1}{\left|\xi_{\alpha} \times \xi_{\beta}\right|}\left(\xi_{\beta} f_{t \alpha}-\xi_{\alpha} f_{t \beta}\right) \\
& =-\left[\partial_{t}, \nabla_{\mathbf{n}}\right] f+\partial_{t}\left(\frac{1}{\left|\xi_{\alpha} \times \xi_{\beta}\right|} \xi_{\beta}\right) f_{\alpha}-\partial_{t}\left(\frac{1}{\left|\xi_{\alpha} \times \xi_{\beta}\right|} \xi_{\alpha}\right) f_{\beta}
\end{aligned}
$$

and

$$
\left[\partial_{t}, H_{\Sigma(t)}^{*}\right]=\mathbf{n}_{t} H_{\Sigma(t)} \mathbf{n}+\mathbf{n}\left[\partial_{t}, H_{\Sigma(t)}\right] \mathbf{n}+\mathbf{n} H_{\Sigma(t)} \mathbf{n}_{t} .
$$

Taking real parts in both sides of (3.18), we get

$$
\begin{aligned}
& -\left[\partial_{t}, \nabla_{\mathbf{n}}\right] f=-\Re\left(\left[\partial_{t}, H_{\Sigma(t)}^{*}\right]\left(\mathbf{n} \mathcal{D}_{\xi} f\right)\right)+\mathcal{K}^{*}\left(\left[\partial_{t}, \nabla_{\mathbf{n}}\right] f\right) \\
& \quad-\int_{\Sigma(t)} \mathbf{n}(\xi) \times K\left(\xi^{\prime}-\xi\right) \cdot\left(\partial_{t}\left(\frac{1}{\left|\xi_{\alpha^{\prime}}^{\prime} \times \xi_{\beta^{\prime}}^{\prime}\right|} \xi_{\beta^{\prime}}\right) f_{\alpha^{\prime}}-\partial_{t}\left(\frac{1}{\left|\xi_{\alpha^{\prime}}^{\prime} \times \xi_{\beta^{\prime}}^{\prime}\right|} \xi_{\alpha^{\prime}}\right) f_{\beta^{\prime}}\right) d S\left(\xi^{\prime}\right) .
\end{aligned}
$$

Therefore

$$
\begin{aligned}
& \left(I+\mathcal{K}^{*}\right)\left(\left[\partial_{t}, \nabla_{\mathbf{n}}\right] f\right) \\
& =\Re\left(-\mathbf{n}_{t} H_{\Sigma(t)}\left(\mathcal{D}_{\xi} f\right)-\mathbf{n}\left[\partial_{t}, H_{\Sigma(t)}\right]\left(\mathcal{D}_{\xi} f\right)+\mathbf{n} H_{\Sigma(t)}\left(\mathbf{n}_{t} \mathbf{n} \mathcal{D}_{\xi} f\right)\right) \\
& +\int_{\Sigma(t)} \mathbf{n}(\xi) \times K\left(\xi^{\prime}-\xi\right) \cdot\left(\partial_{t}\left(\frac{1}{\left|\xi_{\alpha^{\prime}}^{\prime} \times \xi_{\beta^{\prime}}^{\prime}\right|} \xi_{\beta^{\prime}}\right) f_{\alpha^{\prime}}-\partial_{t}\left(\frac{1}{\left|\xi_{\alpha^{\prime}}^{\prime} \times \xi_{\beta^{\prime}}^{\prime}\right|} \xi_{\alpha^{\prime}}\right) f_{\beta^{\prime}}\right) d S\left(\xi^{\prime}\right) .
\end{aligned}
$$

This proves (3.16).

\section{$\S 4$. The equation of Free surfaces and a Key lemma}

We are now ready to study system (1.1)-(1.5). We plan to take a similar approach as the 2-D water wave (see [24], also [6], [18], [25]), that is, we first convert (1.1)-(1.5) into a system on the free surfaces. We then reduce this system to a quasilinear system and solve the quasilinear system, and show that the solution of the quasilinear system is also a solution of the system on the interface.

In this section, we convert system (1.1)-(1.5) into a system on the free surfaces and show that the sign condition (1) always hold for the nonself-intersecting 3D water wave. As we will see, the sign condition (1) is the key that guarantees well-posedness of the quasilinear system in Sobolev spaces.

At time $t$, let $\Omega(t)$ be the water region and let $\Sigma(t)$ be the interface. Suppose $\Sigma(t)$ is described by $\xi(\alpha, \beta, t)=x(\alpha, \beta, t) e_{1}+y(\alpha, \beta, t) e_{2}+z(\alpha, \beta, t) e_{3},-\infty<\alpha, \beta<\infty$, where $(\alpha, \beta)$ is the Lagrangian coordinates, i.e.

$$
\xi_{t}(\alpha, \beta, t)=v(\xi(\alpha, \beta, t), t),
$$


and suppose the unit normal $\mathbf{n}=\frac{\xi_{\alpha} \times \xi_{\beta}}{\left|\xi_{\alpha} \times \xi_{\beta}\right|}$ points out of $\Omega(t)$. From (1.1), we have on the interface, $\xi_{t t}=v_{t}+v \cdot \nabla v=-(0,0,1)-\nabla p$. On the other hand, (1.4) implies that $\nabla p$ is parallel to $\mathbf{n}$. Therefore, $\xi_{t t}+e_{3}=-\nabla p=\mathfrak{a} \mathbf{n}$ (each term in this equation is regarded as a Clifford 1-vector), where

$$
\mathfrak{a}=\left(\xi_{t t}+e_{3}\right) \cdot \mathbf{n}=-\nabla p \cdot \mathbf{n}=-\frac{\partial p}{\partial \mathbf{n}} .
$$

From (1.2), (1.3), we know that the $\mathcal{C}\left(V_{3}\right)$-valued velocity $v(\xi, t)=v_{1}(\xi, t) e_{1}+$ $v_{2}(\xi, t) e_{2}+v_{3}(\xi, t) e_{3}$ is Clifford analytic on $\Omega(t)$, so it's value on $\Sigma(t): v(\xi(\alpha, \beta, t), t)$ $=\xi_{t}(\alpha, \beta, t)$ satisfies (2.10). The initial value problem for system (1.1)-(1.5) is then reduced to the following problem:

$$
\begin{aligned}
& \xi_{t t}+e_{3}=\mathfrak{a} \mathbf{n}, \\
& \xi_{t}=H_{\Sigma(t)} \xi_{t}, \\
& \xi(\alpha, \beta, 0)=\xi_{0}(\alpha, \beta), \xi_{t}(\alpha, \beta, 0)=\xi_{1}(\alpha, \beta),
\end{aligned}
$$

where $\xi=\xi_{0}(\alpha, \beta)$ describes the initial surface $\Sigma(0)$ and $\xi_{1}$ satisfies $\xi_{1}=H_{\Sigma(0)} \xi_{1}$. We will establish the existence and uniqueness of solutions of system (4.3)-(4.5), under the condition that for every $t \geq 0, z(\alpha, \beta, t) \rightarrow 0, \xi_{t}(\alpha, \beta, t) \rightarrow 0$, as $|\alpha|+|\beta| \rightarrow$ $\infty$.

Given a classical solution of (4.3)-(4.5), we can recover the fluid velocity in the whole region $\Omega(t)$ for every fixed time $t$ by solving the Laplace equation:

$$
\begin{array}{ll}
\Delta v=0 & \text { on } \Omega(t), \\
v=\xi_{t} & \text { on } \Sigma(t) .
\end{array}
$$

For the 2-D water wave, we showed that the sign condition (1) always holds for nonself-intersecting interfaces, and (1) was also the key to guaranteeing the wellposedness of the 2-D water wave problem [24]. This suggests that the sign condition (1) will be important and can also be true for the 3 -D water wave. So before we proceed further, we study the quantity $\mathfrak{a}$ defined in (4.2).

Let $\Omega(t)$ be the water region, $\Sigma(t)$ the interface of waterwave at time $t$, and suppose $\Sigma(t)$ is described by $\xi=\xi(\alpha, \beta ; t)$, where $(\alpha, \beta)$ is the Lagrangian parameter, with $\xi_{\alpha} \times \xi_{\beta}$ an outward normal of $\Omega(t)$. We assume that at some time $t_{0}, \Omega\left(t_{0}\right)$ and $\Omega\left(t_{0}\right)^{c}$ are unbounded, simply connected $C^{2}$ domains, and there are constants $C_{0}>0, \mu>0$, and vectors $e_{\alpha}, e_{\beta}$, such that

1. $\left|\xi\left(\alpha, \beta, t_{0}\right)-\xi\left(\alpha^{\prime}, \beta^{\prime}, t_{0}\right)\right| \geq C_{0}\left(\left|\alpha-\alpha^{\prime}\right|+\left|\beta-\beta^{\prime}\right|\right)$, for $-\infty<\alpha, \beta<\infty$;

2. $\xi_{\alpha}\left(\cdot, t_{0}\right)-e_{\alpha}, \xi_{\beta}\left(\cdot, t_{0}\right)-e_{\beta} \in H^{s-1 / 2}\left(R^{2}, \mathcal{C}\left(V_{3}\right)\right), \xi_{t}\left(\cdot, t_{0}\right) \in H^{s+1 / 2}\left(R^{2}, \mathcal{C}\left(V_{3}\right)\right)$, $\xi_{t t}\left(\cdot, t_{0}\right) \in H^{s}\left(R^{2}, \mathcal{C}\left(V_{3}\right)\right)$; and $e_{\alpha} \times e_{\beta}=e_{3}$, for some $s>5 / 2$;

3. $\left|\xi_{\alpha}\left(\cdot, t_{0}\right) \times \xi_{\beta}\left(\cdot, t_{0}\right)\right| \geq \mu$.

We want to prove that the quantity $\mathfrak{a}=-\frac{\partial p}{\partial \mathbf{n}} \geq 2 c_{0}>0$ pointwisely on $\Sigma\left(t_{0}\right)$ for some constant $c_{0}>0$.

Apply div to both sides of (1.1): $v_{t}+v \cdot \nabla v=-\nabla(z+p)$, and use (1.2), (1.3). We get

$$
\operatorname{div}(v \cdot \nabla v)=|\nabla v|^{2}=-\Delta(p+z),
$$

where $|\nabla v|^{2}=\left|\nabla v_{1}\right|^{2}+\left|\nabla v_{2}\right|^{2}+\left|\nabla v_{3}\right|^{2}$. Let $p+z$ be the solution in $\Omega\left(t_{0}\right)$ guaranteed by Theorem 2.5 (notice that $p+z-\frac{1}{2}|v|^{2}$ is harmonic in $\Omega\left(t_{0}\right)$ ). For any $h \in$ $C_{0}^{1}\left(\Sigma\left(t_{0}\right)\right), h \geq 0$, let $w$ be the harmonic extension of $h$ on $\Omega\left(t_{0}\right)$ given by Theorem 
2.5. We know from the remark of Theorem 2.5 that $w \in C^{1}\left(\overline{\Omega\left(t_{0}\right)}\right)$ and $w(\eta)=$ $o\left(|\eta|^{-1}\right), \nabla w(\eta)=o\left(|\eta|^{-2}\right)$ as $|\eta| \rightarrow \infty$. Applying Green's second identity to $w$ and $p+z($ see $[9])$, we get

$$
\int_{\Sigma\left(t_{0}\right)}\left((p+z) \frac{\partial w}{\partial \mathbf{n}}-w \frac{\partial(p+z)}{\partial \mathbf{n}}\right) d S=\int_{\Omega\left(t_{0}\right)} w|\nabla v|^{2} d V
$$

Since $p=0$ on $\Sigma\left(t_{0}\right)((1.4))$, we have

$$
\int_{\Sigma\left(t_{0}\right)} w\left(-\frac{\partial p}{\partial \mathbf{n}}\right) d S=\int_{\Sigma\left(t_{0}\right)}\left(w \frac{\partial z}{\partial \mathbf{n}}-z \frac{\partial w}{\partial \mathbf{n}}\right) d S+\int_{\Omega\left(t_{0}\right)} w|\nabla v|^{2} d V
$$

Let's now calculate the right hand side of (4.6). Assume $\Sigma_{b}: z=b$ is a plane contained in $\Omega\left(t_{0}\right)$, with upward unit normal $\mathbf{n}_{b}=(0,0,1)$. We apply Green's second identity again to $w$ and $z$ on the strip region between surfaces $\Sigma\left(t_{0}\right)$ and $\Sigma_{b}$. Since $w$ and $z$ are harmonic, we have

$$
\begin{aligned}
\int_{\Sigma\left(t_{0}\right)}\left(w \frac{\partial z}{\partial \mathbf{n}}-z \frac{\partial w}{\partial \mathbf{n}}\right) d S & =\lim _{R \rightarrow \infty} \int_{\Sigma_{b} \cap B_{R}(0)}\left(w \frac{\partial z}{\partial \mathbf{n}_{b}}-z \frac{\partial w}{\partial \mathbf{n}_{b}}\right) d S \\
& =\int_{\Sigma_{b}} w \frac{\partial z}{\partial z} d S-b \int_{\Sigma_{b}} \frac{\partial w}{\partial \mathbf{n}_{b}} d S \\
& =\int_{\Sigma_{b}} w d S
\end{aligned}
$$

where $\int_{\Sigma_{b}} \frac{\partial w}{\partial \mathbf{n}_{b}} d S=0$ is obtained from Green's identity, the assumption that $\nabla w(\eta)=o\left(|\eta|^{-2}\right)$ as $|\eta| \rightarrow \infty$ and an approximation argument. Therefore

$$
\begin{aligned}
\int_{\Sigma\left(t_{0}\right)} w\left(-\frac{\partial p}{\partial \mathbf{n}}\right) d S & =\int_{\Sigma_{b}} w d S+\int_{\Omega\left(t_{0}\right)} w|\nabla v|^{2} d V \\
& \geq \int_{\Sigma_{b}} w d S .
\end{aligned}
$$

Let $G=G(\eta, \xi)$ be the (Dirichlet) Green's function for $\Omega\left(t_{0}\right)$. We know from Green's representation formula that

$$
w(\eta)=\int_{\Sigma\left(t_{0}\right)} h(\xi) \frac{\partial G(\eta, \xi)}{\partial \mathbf{n}(\xi)} d S(\xi) \quad \text { for } \eta \in \Omega\left(t_{0}\right) .
$$

Therefore (4.7) implies that

$$
\begin{aligned}
\int_{\Sigma\left(t_{0}\right)} h(\xi)\left(-\frac{\partial p}{\partial \mathbf{n}}(\xi)\right) d S(\xi) & \geq \int_{\Sigma_{b}} w(\eta) d S(\eta) \\
& =\int_{\Sigma\left(t_{0}\right)} h(\xi)\left(\int_{\Sigma_{b}} \frac{\partial G(\eta, \xi)}{\partial \mathbf{n}(\xi)} d S(\eta)\right) d S(\xi) .
\end{aligned}
$$

Since $h \in C_{0}^{1}\left(\Sigma\left(t_{0}\right)\right), h \geq 0$ is arbitrary, we conclude

$$
\mathfrak{a}=-\frac{\partial p}{\partial \mathbf{n}}(\xi) \geq \int_{\Sigma_{b}} \frac{\partial G(\eta, \xi)}{\partial \mathbf{n}(\xi)} d S(\eta)
$$

From the maximal principle, we know there exists a constant $c_{0}>0$, such that $\int_{\Sigma_{b}} \frac{\partial G(\eta, \xi)}{\partial \mathbf{n}(\xi)} d S(\eta) \geq 2 c_{0}$, for all $\xi \in \Sigma\left(t_{0}\right)$.

Remark. Green's identities are justified on unbounded domains here because of the decay properties and the $L^{2}$ integrability of our functions involved. 
Lemma 4.1. Assume that at time $t_{0}$, the water region $\Omega\left(t_{0}\right)$ is an unbounded $C^{2}$ domain, $\Omega\left(t_{0}\right)$ and $\Omega\left(t_{0}\right)^{c}$ are simply connected, and $\Sigma\left(t_{0}\right)$ satisfies assumptions 1 . 2. 3. above. Then

$$
\mathfrak{a}=-\frac{\partial p}{\partial \mathbf{n}}(\xi) \geq \int_{\Sigma_{b}} \frac{\partial G(\eta, \xi)}{\partial \mathbf{n}(\xi)} d S(\eta) \geq 2 c_{0}>0
$$

for all $\xi \in \Sigma\left(t_{0}\right)$, where $\Sigma_{b}: z=b$ is a plane contained in $\Omega\left(t_{0}\right)$, and $c_{0}>0$ is a constant depending only on $\Sigma\left(t_{0}\right)$.

As an immediate consequence of Lemma 4.1 and (4.3), we have

$$
\mathfrak{a}=\left|\xi_{t t}+e_{3}\right|
$$

Remark 1. Our original proof for the 2-D sign condition [24] doesn't apply here, since it uses the Riemann mapping. As we mentioned in $\$ 1$, the idea of expressing $\mathfrak{a}$ as $-\frac{\partial p}{\partial \mathbf{n}}$ and using the maximal principle is from R. Caflisch and T. Hou. The result of Lemma 4.1 shows that for any solutions of the water wave problem, it is necessary that the pressure is positive inside the water region. This is consistent with the physical requirement that pressure is nonnegative.

Remark 2. The prove of Lemma 4.1 is also valid for the 2-D water wave and Lemma 4.1 in the 2-D context coincides with Lemma 3.1 in [24]. In 2-D, the function $\int_{\Sigma_{b}} \frac{\partial G(\eta, \xi)}{\partial \mathbf{n}(\xi)} d S(\eta)$ is the same as the function $h_{\alpha}$ in Lemma 3.1 of [24].

System (4.3)-(4.5) is still a nonlinear, nonlocal system. A standard method for solving such a system is to reduce it to a quasilinear system and solve the quasilinear system. We will do this in the next sections.

\section{$\S 5$. Reduction to A QUASILINEAR SYSTEM}

In this section, we reduce (4.3)-(4.5) to a quasilinear system. Recall the velocity $v=\xi_{t}$ on $\Sigma(t)$ and $v$ is Clifford analytic on $\Omega(t)$. Therefore $\partial_{x} v, \partial_{y} v, \partial_{z} v$ are also Clifford analytic on the same region. Using the notation in (3.9), we have

$$
\partial_{x} \xi_{t}=H_{\Sigma(t)}\left(\partial_{x} \xi_{t}\right), \quad \partial_{y} \xi_{t}=H_{\Sigma(t)}\left(\partial_{y} \xi_{t}\right), \quad \partial_{z} \xi_{t}=H_{\Sigma(t)}\left(\partial_{z} \xi_{t}\right)
$$

Now let's reduce the system (4.3)-(4.4). We use the same notations as in the previous section. Letting $N=\xi_{\alpha} \times \xi_{\beta}$, (4.3) becomes

$$
\xi_{t t}+e_{3}=\frac{\mathfrak{a}}{|N|} N
$$

Taking the derivative with respect to $t$ on both sides of (5.2), we get

$$
\xi_{t t t}=\frac{\mathfrak{a}}{|N|} N_{t}+\left(\frac{\mathfrak{a}}{|N|}\right)_{t} N .
$$


We first calculate $\frac{\mathfrak{a}}{|N|} N_{t}$. Let $N=\left(N_{1}, N_{2}, N_{3}\right)$, and $\mathbf{i}=(1,0,0), \mathbf{j}=(0,1,0)$, $\mathbf{k}=(0,0,1)$. In terms of vector calculus, we have

$$
\begin{aligned}
N_{t} & =\xi_{t \alpha} \times \xi_{\beta}+\xi_{\alpha} \times \xi_{t \beta} \\
& =\left(\xi_{\alpha} \cdot \nabla\right) v \times \xi_{\beta}+\xi_{\alpha} \times\left(\xi_{\beta} \cdot \nabla\right) v \\
& =\mathbf{i} \times\left(\left(\xi_{\alpha} \cdot \nabla v_{1}\right) \xi_{\beta}-\left(\xi_{\beta} \cdot \nabla v_{1}\right) \xi_{\alpha}\right) \\
& +\mathbf{j} \times\left(\left(\xi_{\alpha} \cdot \nabla v_{2}\right) \xi_{\beta}-\left(\xi_{\beta} \cdot \nabla v_{2}\right) \xi_{\alpha}\right) \\
& +\mathbf{k} \times\left(\left(\xi_{\alpha} \cdot \nabla v_{3}\right) \xi_{\beta}-\left(\xi_{\beta} \cdot \nabla v_{3}\right) \xi_{\alpha}\right) \\
& =\mathbf{i} \times\left(\left(\xi_{\alpha} \times \xi_{\beta}\right) \times \nabla v_{1}\right)+\mathbf{j} \times\left(\left(\xi_{\alpha} \times \xi_{\beta}\right) \times \nabla v_{2}\right)+\mathbf{k} \times\left(\left(\xi_{\alpha} \times \xi_{\beta}\right) \times \nabla v_{3}\right) \\
& =\left(\mathbf{i} \cdot \nabla v_{1}+\mathbf{j} \cdot \nabla v_{2}+\mathbf{k} \cdot \nabla v_{3}\right)\left(\xi_{\alpha} \times \xi_{\beta}\right) \\
& -\left(\mathbf{i} \cdot\left(\xi_{\alpha} \times \xi_{\beta}\right)\right) \nabla v_{1}-\left(\mathbf{j} \cdot\left(\xi_{\alpha} \times \xi_{\beta}\right)\right) \nabla v_{2}-\left(\mathbf{k} \cdot\left(\xi_{\alpha} \times \xi_{\beta}\right)\right) \nabla v_{3} \\
& =-N_{1} \partial_{x} v-N_{2} \partial_{y} v-N_{3} \partial_{z} v=-(N \cdot \nabla) v=-\left(N \cdot \nabla_{\xi}\right) \xi_{t}=-|N| \nabla_{\mathbf{n}} \xi_{t} ;
\end{aligned}
$$

here we used the fact that $\mathbf{i} \cdot \nabla v_{1}+\mathbf{j} \cdot \nabla v_{2}+\mathbf{k} \cdot \nabla v_{3}=\partial_{x} v_{1}+\partial_{y} v_{2}+\partial_{z} v_{3}=\operatorname{div} v=0$. Therefore

$$
\frac{\mathfrak{a}}{|N|} N_{t}=-\mathfrak{a} \nabla_{\mathbf{n}} \xi_{t}
$$

To calculate the term $\left(\frac{\mathfrak{a}}{|N|}\right)_{t} N$, we use the relation

$$
\left(\frac{\mathfrak{a}}{|N|}\right)_{t} N=\xi_{t t t}-\frac{\mathfrak{a}}{|N|} N_{t}
$$

Taking the derivative twice with respect to $t$ on both sides of (4.4): $\xi_{t}=H_{\Sigma(t)} \xi_{t}$, we get

$$
\begin{aligned}
\xi_{t t t} & =\partial_{t}^{2}\left(H_{\Sigma(t)} \xi_{t}\right)=\partial_{t}\left(H_{\Sigma(t)} \xi_{t t}+\left[\partial_{t}, H_{\Sigma(t)}\right] \xi_{t}\right) \\
& =H_{\Sigma(t)} \xi_{t t t}+\left[\partial_{t}, H_{\Sigma(t)}\right] \xi_{t t}+\partial_{t}\left(\left[\partial_{t}, H_{\Sigma(t)}\right] \xi_{t}\right)
\end{aligned}
$$

then

$$
\xi_{t t t}-H_{\Sigma(t)} \xi_{t t t}=\left[\partial_{t}, H_{\Sigma(t)}\right] \xi_{t t}+\partial_{t}\left(\left[\partial_{t}, H_{\Sigma(t)}\right] \xi_{t}\right) .
$$

On the other hand, we have from (5.4), (5.2) and (5.1) that

$$
\begin{aligned}
-\frac{\mathfrak{a}}{|N|} N_{t} & =\left(\frac{\mathfrak{a}}{|N|} N \cdot \nabla_{\xi}\right) \xi_{t}=\left(\left(\xi_{t t}+\mathbf{k}\right) \cdot \nabla_{\xi}\right) \xi_{t} \\
& =x_{t t} \partial_{x} \xi_{t}+y_{t t} \partial_{y} \xi_{t}+\left(z_{t t}+1\right) \partial_{z} \xi_{t} \\
& =x_{t t} H_{\Sigma(t)}\left(\partial_{x} \xi_{t}\right)+y_{t t} H_{\Sigma(t)}\left(\partial_{y} \xi_{t}\right)+\left(z_{t t}+1\right) H_{\Sigma(t)}\left(\partial_{z} \xi_{t}\right),
\end{aligned}
$$

therefore

$$
\begin{aligned}
- & \frac{\mathfrak{a}}{|N|} N_{t}-H_{\Sigma(t)}\left(-\frac{\mathfrak{a}}{|N|} N_{t}\right) \\
& =x_{t t} H_{\Sigma(t)}\left(\partial_{x} \xi_{t}\right)+y_{t t} H_{\Sigma(t)}\left(\partial_{y} \xi_{t}\right)+\left(z_{t t}+1\right) H_{\Sigma(t)}\left(\partial_{z} \xi_{t}\right) \\
& -H_{\Sigma(t)}\left(x_{t t} \partial_{x} \xi_{t}+y_{t t} \partial_{y} \xi_{t}+\left(z_{t t}+1\right) \partial_{z} \xi_{t}\right) \\
& =\left[x_{t t}, H_{\Sigma(t)}\right]\left(\partial_{x} \xi_{t}\right)+\left[y_{t t}, H_{\Sigma(t)}\right]\left(\partial_{y} \xi_{t}\right)+\left[z_{t t}, H_{\Sigma(t)}\right]\left(\partial_{z} \xi_{t}\right) .
\end{aligned}
$$


Adding (5.8) to (5.7), we obtain

$$
\begin{aligned}
& \left(\frac{\mathfrak{a}}{|N|}\right)_{t} N-H_{\Sigma(t)}\left(\left(\frac{\mathfrak{a}}{|N|}\right)_{t} N\right) \\
& =\xi_{t t t}-H_{\Sigma(t)} \xi_{t t t}-\frac{\mathfrak{a}}{|N|} N_{t}-H_{\Sigma(t)}\left(-\frac{\mathfrak{a}}{|N|} N_{t}\right) \\
& =\left[\partial_{t}, H_{\Sigma(t)}\right] \xi_{t t}+\partial_{t}\left(\left[\partial_{t}, H_{\Sigma(t)}\right] \xi_{t}\right) \\
& +\left[x_{t t}, H_{\Sigma(t)}\right]\left(\partial_{x} \xi_{t}\right)+\left[y_{t t}, H_{\Sigma(t)}\right]\left(\partial_{y} \xi_{t}\right)+\left[z_{t t}, H_{\Sigma(t)}\right]\left(\partial_{z} \xi_{t}\right) .
\end{aligned}
$$

We want to single out $\left(\frac{\mathfrak{a}}{|N|}\right)_{t} N$. Notice that $\mathbf{n}\left(\frac{\mathfrak{a}}{|N|}\right)_{t} N=-\left(\frac{\mathfrak{a}}{|N|}\right)_{t}|N|$ is a real-valued function, and $\mathbf{n} H_{\Sigma(t)}=-H_{\Sigma(t)}^{*} \mathbf{n}$; we multiply by $-\mathbf{n}$ at the left to both sides of (5.9), then take the real part. We get on the left hand side

$$
\begin{aligned}
& \Re\left\{-\mathbf{n}\left\{\left(\frac{\mathfrak{a}}{|N|}\right)_{t} N-H_{\Sigma(t)}\left(\left(\frac{\mathfrak{a}}{|N|}\right)_{t} N\right)\right\}\right\} \\
& =\left(\frac{\mathfrak{a}}{|N|}\right)_{t}|N|+\Re\left\{H_{\Sigma(t)}^{*}\left(\left(\frac{\mathfrak{a}}{|N|}\right)_{t}|N|\right)\right\} \\
& =\left(\frac{\mathfrak{a}}{|N|}\right)_{t}|N|+\mathcal{K}^{*}\left(\left(\frac{\mathfrak{a}}{|N|}\right)_{t}|N|\right)_{t} \\
& =\left(I+\mathcal{K}^{*}\right)\left(\left(\frac{\mathfrak{a}}{|N|}\right)_{t}|N|\right),
\end{aligned}
$$

therefore

$$
\begin{aligned}
\left(I+\mathcal{K}^{*}\right)\left(\left(\frac{\mathfrak{a}}{|N|}\right)_{t}|N|\right)=-\Re\left(\mathbf { n } \left\{\left[\partial_{t}, H_{\Sigma(t)}\right] \xi_{t t}+\partial_{t}\left(\left[\partial_{t}, H_{\Sigma(t)}\right] \xi_{t}\right)\right.\right. \\
\left.\left.\quad+\left[x_{t t}, H_{\Sigma(t)}\right]\left(\partial_{x} \xi_{t}\right)+\left[y_{t t}, H_{\Sigma(t)}\right]\left(\partial_{y} \xi_{t}\right)+\left[z_{t t}, H_{\Sigma(t)}\right]\left(\partial_{z} \xi_{t}\right)\right\}\right) .
\end{aligned}
$$

Consequently,

$$
\left(\frac{\mathfrak{a}}{|N|}\right)_{t} N=\left(\frac{\mathfrak{a}}{|N|}\right)_{t}|N| \mathbf{n}=-\mathbf{n}\left(I+\mathcal{K}^{*}\right)^{-1}\left(\Re\left\{\mathbf{n} f\left(\xi, \xi_{t}, \xi_{t t}\right)\right\}\right)
$$

where

$$
\begin{aligned}
f\left(\xi, \xi_{t}, \xi_{t t}\right) & =\left[\partial_{t}, H_{\Sigma(t)}\right] \xi_{t t}+\partial_{t}\left(\left[\partial_{t}, H_{\Sigma(t)}\right] \xi_{t}\right) \\
& +\left[x_{t t}, H_{\Sigma(t)}\right]\left(\partial_{x} \xi_{t}\right)+\left[y_{t t}, H_{\Sigma(t)}\right]\left(\partial_{y} \xi_{t}\right)+\left[z_{t t}, H_{\Sigma(t)}\right]\left(\partial_{z} \xi_{t}\right) .
\end{aligned}
$$

Placing the highest order terms to the left, we get from (5.2), (5.5), (5.11) the following equation:

$$
\xi_{t t t}+\mathfrak{a} \nabla_{\mathbf{n}} \xi_{t}=-\mathbf{n}\left(I+\mathcal{K}^{*}\right)^{-1}\left(\Re\left\{\mathbf{n} f\left(\xi, \xi_{t}, \xi_{t t}\right)\right\}\right),
$$

where $\mathfrak{a}=\left|\xi_{t t}+e_{3}\right|, \mathbf{n}=\frac{\xi_{t t}+e_{3}}{\left|\xi_{t t}+e_{3}\right|}$, and $f\left(\xi, \xi_{t}, \xi_{t t}\right)$ is as given in (5.12). Now let's calculate $f\left(\xi, \xi_{t}, \xi_{t t}\right)$. Recall that we have calculated the commutator $\left[\partial_{t}, H_{\Sigma(t)}\right] f$ in Lemma 3.1 for the scalar-valued function $f$. Applying (3.2) to each component of $\xi_{t t}$ then multiplying $e_{j}$ to the right and adding them up, we obtain

$$
\begin{aligned}
{\left[\partial_{t}, H_{\Sigma(t)}\right] \xi_{t t}=p . v . \iint K\left(\xi^{\prime}-\xi\right)\left\{\left(\left(\xi_{t}-\xi_{t}^{\prime}\right) \times \xi_{\beta^{\prime}}^{\prime}\right) \xi_{t t \alpha^{\prime}}^{\prime}\right.} \\
\left.-\left(\left(\xi_{t}-\xi_{t}^{\prime}\right) \times \xi_{\alpha^{\prime}}^{\prime}\right) \xi_{t t \beta^{\prime}}^{\prime}\right\} d \alpha^{\prime} d \beta^{\prime}
\end{aligned}
$$


similarly

$$
\begin{aligned}
{\left[\partial_{t}, H_{\Sigma(t)}\right] \xi_{t}=p \cdot v \cdot \iint K\left(\xi^{\prime}-\xi\right)\left\{\left(\left(\xi_{t}-\xi_{t}^{\prime}\right) \times \xi_{\beta^{\prime}}^{\prime}\right) \xi_{t \alpha^{\prime}}^{\prime}\right.} \\
\left.-\left(\left(\xi_{t}-\xi_{t}^{\prime}\right) \times \xi_{\alpha^{\prime}}^{\prime}\right) \xi_{t \beta^{\prime}}^{\prime}\right\} d \alpha^{\prime} d \beta^{\prime} ;
\end{aligned}
$$

then

$$
\begin{aligned}
\partial_{t} & \left(\left[\partial_{t}, H_{\Sigma(t)}\right] \xi_{t}\right) \\
& =p . v \cdot \iint K\left(\xi^{\prime}-\xi\right)\left\{\left(\left(\xi_{t t}-\xi_{t t}^{\prime}\right) \times \xi_{\beta^{\prime}}^{\prime}\right) \xi_{t \alpha^{\prime}}^{\prime}-\left(\left(\xi_{t t}-\xi_{t t}^{\prime}\right) \times \xi_{\alpha^{\prime}}^{\prime}\right) \xi_{t \beta^{\prime}}^{\prime}\right\} d \alpha^{\prime} d \beta^{\prime} \\
& +p . v \cdot \iint K\left(\xi^{\prime}-\xi\right)\left\{\left(\left(\xi_{t}-\xi_{t}^{\prime}\right) \times \xi_{t \beta^{\prime}}^{\prime}\right) \xi_{t \alpha^{\prime}}^{\prime}-\left(\left(\xi_{t}-\xi_{t}^{\prime}\right) \times \xi_{t \alpha^{\prime}}^{\prime}\right) \xi_{t \beta^{\prime}}^{\prime}\right\} d \alpha^{\prime} d \beta^{\prime} \\
& + \text { p.v. } \iint K\left(\xi^{\prime}-\xi\right)\left\{\left(\left(\xi_{t}-\xi_{t}^{\prime}\right) \times \xi_{\beta^{\prime}}^{\prime}\right) \xi_{t t \alpha^{\prime}}^{\prime}-\left(\left(\xi_{t}-\xi_{t}^{\prime}\right) \times \xi_{\alpha^{\prime}}^{\prime}\right) \xi_{t t \beta^{\prime}}^{\prime}\right\} d \alpha^{\prime} d \beta^{\prime} \\
& +p . v \cdot \iint \partial_{t}\left\{K\left(\xi^{\prime}-\xi\right)\right\}\left\{\left(\left(\xi_{t}-\xi_{t}^{\prime}\right) \times \xi_{\beta^{\prime}}^{\prime}\right) \xi_{t \alpha^{\prime}}^{\prime}-\left(\left(\xi_{t}-\xi_{t}^{\prime}\right) \times \xi_{\alpha^{\prime}}^{\prime}\right) \xi_{t \beta^{\prime}}^{\prime}\right\} d \alpha^{\prime} d \beta^{\prime} .
\end{aligned}
$$

We want to further reduce the last term in (5.16). Since for any vector $\eta$,

$$
\begin{aligned}
& \left(\eta \times \xi_{\beta}\right) \xi_{t \alpha}-\left(\eta \times \xi_{\alpha}\right) \xi_{t \beta}=\eta \times\left\{\xi_{\beta}\left(\xi_{\alpha} \cdot \nabla_{\xi}\right)-\xi_{\alpha}\left(\xi_{\beta} \cdot \nabla_{\xi}\right)\right\} \xi_{t} \\
& =\eta \times\left\{\left(\xi_{\alpha} \times \xi_{\beta}\right) \times \nabla_{\xi}\right\} \xi_{t}=\left(\xi_{\alpha} \times \xi_{\beta}\right)\left(\eta \cdot \nabla_{\xi}\right) \xi_{t}-\left(\eta \cdot\left(\xi_{\alpha} \times \xi_{\beta}\right)\right) \mathcal{D}_{\xi} \xi_{t} \\
& =\left(\xi_{\alpha} \times \xi_{\beta}\right)\left(\eta \cdot \nabla_{\xi}\right) \xi_{t},
\end{aligned}
$$

where in the last equality we used the fact that $v$ is Clifford analytic on $\Omega(t)$, that is, $\mathcal{D}_{\xi} \xi_{t}=\mathcal{D} v(\xi)=0$, we get, using (5.17) and (3.5), and integration by parts, that

$$
\begin{aligned}
& \text { p.v. } \iint \partial_{t}\left\{K\left(\xi^{\prime}-\xi\right)\right\}\left\{\left(\left(\xi_{t}-\xi_{t}^{\prime}\right) \times \xi_{\beta^{\prime}}^{\prime}\right) \xi_{t \alpha^{\prime}}^{\prime}-\left(\left(\xi_{t}-\xi_{t}^{\prime}\right) \times \xi_{\alpha^{\prime}}^{\prime}\right) \xi_{t \beta^{\prime}}^{\prime}\right\} d \alpha^{\prime} d \beta^{\prime} \\
& =p . v \cdot \iint \partial_{t}\left\{K\left(\xi^{\prime}-\xi\right)\right\}\left(\xi_{\alpha^{\prime}}^{\prime} \times \xi_{\beta^{\prime}}^{\prime}\right)\left(\left(\xi_{t}-\xi_{t}^{\prime}\right) \cdot \nabla_{\xi^{\prime}}\right) \xi_{t}^{\prime} d \alpha^{\prime} d \beta^{\prime} \\
& =p . v \cdot \iint\left\{\partial_{\alpha^{\prime}} K\left(\left(\xi_{t}^{\prime}-\xi_{t}\right) \times \xi_{\beta^{\prime}}^{\prime}\right)+\partial_{\beta^{\prime}} K\left(\xi_{\alpha^{\prime}}^{\prime} \times\left(\xi_{t}^{\prime}-\xi_{t}\right)\right)\right\}\left(\left(\xi_{t}-\xi_{t}^{\prime}\right) \cdot \nabla_{\xi^{\prime}}\right) \xi_{t}^{\prime} d \alpha^{\prime} d \beta^{\prime} \\
& =-p \cdot v \cdot \iint K\left(\xi^{\prime}-\xi\right)\left(\xi_{t}^{\prime}-\xi_{t}\right) \times \xi_{\beta^{\prime}}^{\prime}\left\{\left(\xi_{t}-\xi_{t}^{\prime}\right) \cdot \partial_{\alpha^{\prime}} \nabla_{\xi^{\prime}}\right\} \xi_{t}^{\prime} d \alpha^{\prime} d \beta^{\prime} \\
& -p . v \cdot \iint K\left(\xi^{\prime}-\xi\right) \xi_{\alpha^{\prime}}^{\prime} \times\left(\xi_{t}^{\prime}-\xi_{t}\right)\left\{\left(\xi_{t}-\xi_{t}^{\prime}\right) \cdot \partial_{\beta^{\prime}} \nabla_{\xi^{\prime}}\right\} \xi_{t}^{\prime} d \alpha^{\prime} d \beta^{\prime} \\
& +p \cdot v \cdot \iint K\left(\xi^{\prime}-\xi\right)\left\{\left(\xi_{t}^{\prime}-\xi_{t}\right) \times \xi_{\beta^{\prime}}^{\prime}\left(\xi_{t \alpha^{\prime}}^{\prime} \cdot \nabla_{\xi^{\prime}}\right) \xi_{t}^{\prime}\right. \\
& \left.\quad+\xi_{\alpha^{\prime}}^{\prime} \times\left(\xi_{t}^{\prime}-\xi_{t}\right)\left(\xi_{t \beta^{\prime}}^{\prime} \cdot \nabla_{\xi^{\prime}}\right) \xi_{t}^{\prime}\right\} d \alpha^{\prime} d \beta^{\prime} \\
& -p . v \cdot \iint K\left(\xi^{\prime}-\xi\right)\left(\xi_{t \alpha^{\prime}}^{\prime} \times \xi_{\beta^{\prime}}^{\prime}+\xi_{\alpha^{\prime}}^{\prime} \times \xi_{t \beta^{\prime}}^{\prime}\right)\left(\left(\xi_{t}-\xi_{t}^{\prime}\right) \cdot \nabla_{\xi^{\prime}}\right) \xi_{t}^{\prime} d \alpha^{\prime} d \beta^{\prime} .
\end{aligned}
$$


We also have from (5.17) that

$$
\begin{aligned}
{\left[x_{t t},\right.} & \left.H_{\Sigma(t)}\right]\left(\partial_{x} \xi_{t}\right)+\left[y_{t t}, H_{\Sigma(t)}\right]\left(\partial_{y} \xi_{t}\right)+\left[z_{t t}, H_{\Sigma(t)}\right]\left(\partial_{z} \xi_{t}\right) \\
& =p . v \cdot \iint K\left(\xi^{\prime}-\xi\right)\left(\xi_{\alpha}^{\prime} \times \xi_{\beta}^{\prime}\right)\left(\left(\xi_{t t}-\xi_{t t}^{\prime}\right) \cdot \nabla_{\xi^{\prime}}\right) \xi_{t}^{\prime} d \alpha^{\prime} d \beta^{\prime} \\
& =p . v \cdot \iint K\left(\xi^{\prime}-\xi\right)\left\{\left(\left(\xi_{t t}-\xi_{t t}^{\prime}\right) \times \xi_{\beta^{\prime}}^{\prime}\right) \xi_{t \alpha^{\prime}}^{\prime}-\left(\left(\xi_{t t}-\xi_{t t}^{\prime}\right) \times \xi_{\alpha^{\prime}}^{\prime}\right) \xi_{t \beta^{\prime}}^{\prime}\right\} d \alpha^{\prime} d \beta^{\prime}
\end{aligned}
$$

Therefore

$$
\begin{aligned}
& f\left(\xi, \xi_{t}, \xi_{t t}\right) \\
& \quad=2 p \cdot v \cdot \iint K\left(\xi^{\prime}-\xi\right)\left\{\left(\left(\xi_{t t}-\xi_{t t}^{\prime}\right) \times \xi_{\beta^{\prime}}^{\prime}\right) \xi_{t \alpha^{\prime}}^{\prime}-\left(\left(\xi_{t t}-\xi_{t t}^{\prime}\right) \times \xi_{\alpha^{\prime}}^{\prime}\right) \xi_{t \beta^{\prime}}^{\prime}\right\} d \alpha^{\prime} d \beta^{\prime} \\
& \quad+p . v \cdot \iint K\left(\xi^{\prime}-\xi\right)\left\{\left(\left(\xi_{t}-\xi_{t}^{\prime}\right) \times \xi_{t \beta^{\prime}}^{\prime}\right) \xi_{t \alpha^{\prime}}^{\prime}-\left(\left(\xi_{t}-\xi_{t}^{\prime}\right) \times \xi_{t \alpha^{\prime}}^{\prime}\right) \xi_{t \beta^{\prime}}^{\prime}\right\} d \alpha^{\prime} d \beta^{\prime} \\
& \quad+2 p . v \cdot \iint K\left(\xi^{\prime}-\xi\right)\left\{\left(\left(\xi_{t}-\xi_{t}^{\prime}\right) \times \xi_{\beta^{\prime}}^{\prime}\right) \xi_{t t \alpha^{\prime}}^{\prime}-\left(\left(\xi_{t}-\xi_{t}^{\prime}\right) \times \xi_{\alpha^{\prime}}^{\prime}\right) \xi_{t t \beta^{\prime}}^{\prime}\right\} d \alpha^{\prime} d \beta^{\prime} \\
& \quad-\text { p.v. } \iint K\left(\xi^{\prime}-\xi\right)\left(\xi_{t}^{\prime}-\xi_{t}\right) \times \xi_{\beta^{\prime}}^{\prime}\left\{\left(\xi_{t}-\xi_{t}^{\prime}\right) \cdot \partial_{\alpha^{\prime}} \nabla_{\xi^{\prime}}\right\} \xi_{t}^{\prime} d \alpha^{\prime} d \beta^{\prime} \\
& \quad-p . v \cdot \iint K\left(\xi^{\prime}-\xi\right) \xi_{\alpha^{\prime}}^{\prime} \times\left(\xi_{t}^{\prime}-\xi_{t}\right)\left\{\left(\xi_{t}-\xi_{t}^{\prime}\right) \cdot \partial_{\beta^{\prime}} \nabla_{\xi^{\prime}}\right\} \xi_{t}^{\prime} d \alpha^{\prime} d \beta^{\prime} \\
& \quad+p . v \cdot \iint K\left(\xi^{\prime}-\xi\right)\left\{\left(\xi_{t}^{\prime}-\xi_{t}\right) \times \xi_{\beta^{\prime}}^{\prime}\left(\xi_{t \alpha^{\prime}}^{\prime} \cdot \nabla_{\xi^{\prime}}\right) \xi_{t}^{\prime}\right. \\
& \left.\quad+\xi_{\alpha^{\prime}}^{\prime} \times\left(\xi_{t}^{\prime}-\xi_{t}\right)\left(\xi_{t \beta^{\prime}}^{\prime} \cdot \nabla_{\xi^{\prime}}\right) \xi_{t}^{\prime}\right\} d \alpha^{\prime} d \beta^{\prime} \\
& \quad-p . v . \iint K\left(\xi^{\prime}-\xi\right)\left(\xi_{t \alpha^{\prime}}^{\prime} \times \xi_{\beta^{\prime}}^{\prime}+\xi_{\alpha^{\prime}}^{\prime} \times \xi_{t \beta^{\prime}}^{\prime}\right)\left(\left(\xi_{t}-\xi_{t}^{\prime}\right) \cdot \nabla_{\xi^{\prime}}\right) \xi_{t}^{\prime} d \alpha^{\prime} d \beta^{\prime} .
\end{aligned}
$$

So we convert (4.3)-(4.4) into the following quasilinear equation: Suppose $\xi=$ $\xi(\alpha, \beta ; t)$ is a solution of (4.3)-(4.4). Let $\mathfrak{u}=\xi_{t}$. Then $\mathfrak{u}$ is necessarily a solution of the quasilinear equation

$$
\mathfrak{u}_{t t}+\mathfrak{a} \nabla_{\mathbf{n}} \mathfrak{u}=-\tilde{\mathbf{n}}\left(I+\widetilde{\mathcal{K}^{*}}\right)^{-1}\left(\Re\left\{\tilde{\mathbf{n}} f\left(\xi, \mathfrak{u}, \mathfrak{u}_{t}\right)\right\}\right),
$$

where

$$
\begin{aligned}
\mathfrak{a} & =\left|\mathfrak{u}_{t}+e_{3}\right|, \quad \tilde{\mathbf{n}}=\frac{\mathfrak{u}_{t}+e_{3}}{\left|\mathfrak{u}_{t}+e_{3}\right|}, \\
\xi(\alpha, \beta, t) & =\xi(\alpha, \beta, 0)+\int_{0}^{t} \mathfrak{u}(\alpha, \beta, s) d s, \quad \mathfrak{U}=\frac{1}{2}\left(\mathfrak{u}+H_{\Sigma(t)} \mathfrak{u}\right),
\end{aligned}
$$




$$
\begin{aligned}
f\left(\xi, \mathfrak{u}, \mathfrak{u}_{t}\right) & =2 p \cdot v \cdot \iint K\left(\xi^{\prime}-\xi\right)\left\{\left(\left(\mathfrak{u}_{t}-\mathfrak{u}_{t}^{\prime}\right) \times \xi_{\beta^{\prime}}^{\prime}\right) \mathfrak{U}_{\alpha^{\prime}}^{\prime}-\left(\left(\mathfrak{u}_{t}-\mathfrak{u}_{t}^{\prime}\right) \times \xi_{\alpha^{\prime}}^{\prime}\right) \mathfrak{U}_{\beta^{\prime}}^{\prime}\right\} d \alpha^{\prime} d \beta^{\prime} \\
& + \text { p.v. } \iint K\left(\xi^{\prime}-\xi\right)\left\{\left(\left(\mathfrak{u}-\mathfrak{u}^{\prime}\right) \times \mathfrak{u}_{\beta^{\prime}}^{\prime}\right) \mathfrak{U}_{\alpha^{\prime}}^{\prime}-\left(\left(\mathfrak{u}-\mathfrak{u}^{\prime}\right) \times \mathfrak{u}_{\alpha^{\prime}}^{\prime}\right) \mathfrak{U}_{\beta^{\prime}}^{\prime}\right\} d \alpha^{\prime} d \beta^{\prime} \\
& +2 p . v \cdot \iint K\left(\xi^{\prime}-\xi\right)\left\{\left(\left(\mathfrak{u}-\mathfrak{u}^{\prime}\right) \times \xi_{\beta^{\prime}}^{\prime}\right) \mathfrak{U}_{t \alpha^{\prime}}^{\prime}-\left(\left(\mathfrak{u}-\mathfrak{u}^{\prime}\right) \times \xi_{\alpha^{\prime}}^{\prime}\right) \mathfrak{U}_{\beta^{\prime}}^{\prime}\right\} d \alpha^{\prime} d \beta^{\prime} \\
& + \text { p.v. } \iint K\left(\xi^{\prime}-\xi\right)\left(\mathfrak{u}-\mathfrak{u}^{\prime}\right) \times \xi_{\beta^{\prime}}^{\prime}\left\{\left(\mathfrak{u}-\mathfrak{u}^{\prime}\right) \cdot \partial_{\alpha^{\prime}} \nabla_{\xi^{\prime}}\right\} \mathfrak{U}^{\prime} d \alpha^{\prime} d \beta^{\prime} \\
& + \text { p.v. } \iint K\left(\xi^{\prime}-\xi\right) \xi_{\alpha^{\prime}}^{\prime} \times\left(\mathfrak{u}-\mathfrak{u}^{\prime}\right)\left\{\left(\mathfrak{u}-\mathfrak{u}^{\prime}\right) \cdot \partial_{\beta^{\prime}} \nabla_{\xi^{\prime}}\right\} \mathfrak{U}^{\prime} d \alpha^{\prime} d \beta^{\prime} \\
& -p . v \cdot \iint K\left(\xi^{\prime}-\xi\right)\left\{\left(\mathfrak{u}-\mathfrak{u}^{\prime}\right) \times \xi_{\beta^{\prime}}^{\prime}\left(\mathfrak{u}_{\alpha^{\prime}}^{\prime} \cdot \nabla_{\xi^{\prime}}\right) \mathfrak{U}^{\prime}\right. \\
& \left.+\xi_{\alpha^{\prime}}^{\prime} \times\left(\mathfrak{u}-\mathfrak{u}^{\prime}\right)\left(\mathfrak{u}_{\beta^{\prime}}^{\prime} \cdot \nabla_{\xi^{\prime}}\right) \mathfrak{U}^{\prime}\right\} d \alpha^{\prime} d \beta^{\prime} \\
& -p . v \cdot \iint K\left(\xi^{\prime}-\xi\right)\left(\mathfrak{u}_{\alpha^{\prime}}^{\prime} \times \xi_{\beta^{\prime}}^{\prime}+\xi_{\alpha^{\prime}}^{\prime} \times \mathfrak{u}_{\beta^{\prime}}^{\prime}\right)\left(\left(\mathfrak{u}-\mathfrak{u}^{\prime}\right) \cdot \nabla_{\xi^{\prime}}\right) \mathfrak{U}^{\prime} d \alpha^{\prime} d \beta^{\prime} ;
\end{aligned}
$$

here $\nabla_{\mathbf{n}} \mathfrak{u}$ is the normal derivative of $\mathfrak{u}, \nabla_{\xi} \mathfrak{u}$ is the space variable gradient of $\mathfrak{u}$ and $H_{\Sigma(t)} \mathfrak{u}$ is the Hilbert transform of $\mathfrak{u}$ with respect to the surface $\Sigma(t): \xi=\xi(\alpha, \beta, t)$ defined in (5.22) and the lower region $\Omega(t)$ bounded by $\Sigma(t)$. For the real-valued function $g$,

$$
\widetilde{\mathcal{K}^{*}} g=\Re\left\{\tilde{\mathbf{n}} H_{\Sigma(t)}(\tilde{\mathbf{n}} g)\right\} .
$$

Notice that a solution $\mathfrak{u}$ of (5.21)-(5.23) is not obviously analytic, and does not obviously give $\tilde{\mathbf{n}}=\mathbf{n}$. In $\S 7$, we will show that a solution of (5.21)-(5.23) with proper initial data is analytic and gives rise to a solution of (4.3)-(4.4).

Initial data. We need to transform the initial data (4.5) into an initial data for (5.21)-(5.23). We will do so by first finding a formula expressing $\mathfrak{a}$, consequently expressing $\xi_{t t}$, as a function of $\xi_{t}$ and $\xi$. Since $\xi_{t t}+e_{3}=\mathfrak{a} \mathbf{n}$, we have

$$
\mathfrak{a}=-\mathbf{n}\left(\xi_{t t}+e_{3}\right),
$$

therefore

$$
\mathfrak{a}-\mathbf{n} \cdot e_{3}=-\mathbf{n} \xi_{t t}-\mathbf{n} \times e_{3} .
$$

Now taking the derivative with respect to $t$ on both sides of (4.4), we get $\xi_{t t}=$ $\partial_{t}\left(H_{\Sigma(t)} \xi_{t}\right)=H_{\Sigma(t)} \xi_{t t}+\left[\partial_{t}, H_{\Sigma(t)}\right] \xi_{t}$, therefore

$$
\xi_{t t}-H_{\Sigma(t)} \xi_{t t}=\left[\partial_{t}, H_{\Sigma(t)}\right] \xi_{t} .
$$

Consequently

$$
\mathbf{n} \xi_{t t}+H_{\Sigma(t)}^{*}\left(\mathbf{n} \xi_{t t}\right)=\mathbf{n} \xi_{t t}-\mathbf{n} H_{\Sigma(t)} \xi_{t t}=\mathbf{n}\left[\partial_{t}, H_{\Sigma(t)}\right] \xi_{t} .
$$

From (5.24) and the fact that $\mathfrak{a}-\mathbf{n} \cdot e_{3}$ is a real-valued function, we have

$$
\begin{aligned}
\left(I+\mathcal{K}^{*}\right)\left(\mathfrak{a}-\mathbf{n} \cdot e_{3}\right) & =-\Re\left\{\left(I+H_{\Sigma(t)}^{*}\right)\left(\mathbf{n} \xi_{t t}+\mathbf{n} \times e_{3}\right)\right\} \\
& =-\Re\left\{\mathbf{n}\left[\partial_{t}, H_{\Sigma(t)}\right] \xi_{t}+H_{\Sigma(t)}^{*}\left(\mathbf{n} \times e_{3}\right)\right\}
\end{aligned}
$$

So

$$
\mathfrak{a}=\mathbf{n} \cdot e_{3}-\left(I+\mathcal{K}^{*}\right)^{-1}\left(\Re\left\{\mathbf{n}\left[\partial_{t}, H_{\Sigma(t)}\right] \xi_{t}+H_{\Sigma(t)}^{*}\left(\mathbf{n} \times e_{3}\right)\right\}\right)
$$


where $\left[\partial_{t}, H_{\Sigma(t)}\right] \xi_{t}$ is as given in (5.15). Since $\xi_{t t}+e_{3}=\mathfrak{a n}$, we have

$$
\xi_{t t}=-e_{3}+\left(\mathbf{n} \cdot e_{3}\right) \mathbf{n}-\mathbf{n}\left(I+\mathcal{K}^{*}\right)^{-1}\left(\Re\left\{\mathbf{n}\left[\partial_{t}, H_{\Sigma(t)}\right] \xi_{t}+H_{\Sigma(t)}^{*}\left(\mathbf{n} \times e_{3}\right)\right\}\right) .
$$

We can now determine the initial data of (5.21)-(5.23). Assume that the initial surface $\Sigma(0)$ divides $R^{3}$ into two simply connected, unbounded $C^{2}$ domains, $\Sigma(0)$ approaches the $x y$-plane at infinity, and assume water occupies the lower domain $\Omega(0)$. Without loss of generality, we choose the parameterization of $\Sigma(0): \xi=$ $\xi_{0}(\alpha, \beta),-\infty<\alpha, \beta<\infty$, such that $\xi_{0, \alpha} \times \xi_{0, \beta}$ is an outer normal of $\Omega(0)$,

$$
\left|\xi_{0, \alpha} \times \xi_{0, \beta}\right| \geq 2 \mu, \quad-\infty<\alpha, \beta<\infty,
$$

for some constant $\mu>0$, and for some unit vectors $e_{\alpha}, e_{\beta}$, with $e_{\alpha} \times e_{\beta}=e_{3}$, $\xi_{0, \alpha}-e_{\alpha} \rightarrow 0, \xi_{0, \beta}-e_{\beta} \rightarrow 0$ as $|\alpha|+|\beta| \rightarrow \infty$. For this parameterization, we know that there is a constant $C_{0}>0$, such that

$$
\left|\xi_{0}(\alpha, \beta)-\xi_{0}\left(\alpha^{\prime}, \beta^{\prime}\right)\right| \geq 2 C_{0}\left(\left|\alpha-\alpha^{\prime}\right|+\left|\beta-\beta^{\prime}\right|\right), \quad \text { for }-\infty<\alpha, \beta<\infty .
$$

Let

$$
\xi(\alpha, \beta, 0)=\xi_{0}(\alpha, \beta), \quad \xi_{t}(\alpha, \beta, 0)=\xi_{1}(\alpha, \beta)
$$

be the initial data for (4.3)-(4.4), where $\xi_{1}$ satisfies $\xi_{1}=H_{\Sigma(0)} \xi_{1}$. We take the corresponding initial data for (5.21)-(5.23)

$$
\mathfrak{u}(\alpha, \beta, 0)=\mathfrak{u}_{0}(\alpha, \beta), \quad \mathfrak{u}_{t}(\alpha, \beta, 0)=\mathfrak{u}_{1}(\alpha, \beta)
$$

such that $\mathfrak{u}_{0}=\xi_{1}$, and

$$
\mathfrak{u}_{1}=-e_{3}+\left(\mathbf{n}_{0} \cdot e_{3}\right) \mathbf{n}_{0}-\mathbf{n}_{0}\left(I+\mathcal{K}_{0}^{*}\right)^{-1}\left(\Re\left\{\mathbf{n}_{0}\left[\partial_{t}, H_{\Sigma(0)}\right] \mathfrak{u}_{0}+H_{\Sigma(0)}^{*}\left(\mathbf{n}_{0} \times e_{3}\right)\right\}\right)
$$

where $\mathbf{n}_{0}$ is the outer unit normal of $\Omega(0), \mathcal{K}_{0}^{*}$ is the adjoint of the double layer potential defined by $\Sigma(0)$ and lower region $\Omega(0)$, and

$$
\begin{aligned}
{\left[\partial_{t}, H_{\Sigma(0)}\right] \mathfrak{u}_{0}=p . v . \iint K\left(\xi^{\prime}-\xi\right)\{} & \left(\left(\mathfrak{u}_{0}-\mathfrak{u}_{0}^{\prime}\right) \times \xi_{0, \beta^{\prime}}^{\prime}\right) \mathfrak{u}_{0, \alpha^{\prime}}^{\prime} \\
& \left.-\left(\left(\mathfrak{u}_{0}-\mathfrak{u}_{0}^{\prime}\right) \times \xi_{0, \alpha^{\prime}}^{\prime}\right) \mathfrak{u}_{0, \beta^{\prime}}^{\prime}\right\} d \alpha^{\prime} d \beta^{\prime} .
\end{aligned}
$$

We know $\mathbf{n}_{0}=\frac{\mathfrak{u}_{1}+e_{3}}{\left|\mathfrak{u}_{1}+e_{3}\right|}$. If $\Sigma(0)$ further satisfies the assumption 2. of Lemma 4.1, we have that

$$
\mathfrak{a}_{0}=\left|\mathfrak{u}_{1}+e_{3}\right| \geq 2 c_{0}
$$

for some positive constant $c_{0}$.

In the next section, we will study the well-posedness of the quasilinear system (5.21)-(5.23), with initial data (5.29) satisfying (5.27), (5.28), (4.5'), (5.30) and $(5.31)$.

\section{§6. WELL-POSEDNESS OF THE QUASILINEAR SYSTEM}

In this section, we show that the quasilinear system (5.21)-(5.23),(5.29) is uniquely solvable in certain Sobolev spaces. Our approach is standard. That is we first solve the linear system defined on a given collection of surfaces $\Sigma(t), t \in[0, T]$, and then use iteration to solve the quasilinear system. Solutions of both the linear and quasilinear systems are obtained by proper energy estimates, iteration and the fixed point theorem. This approach is very much the same as in the $2-\mathrm{D}$ case; see 
[24], also [25]. The main difference is that in [24], we used Riemann mapping to flatten out the interface, therefore the estimates there are in clean and compact forms, while here we solve (5.21)-(5.23) directly. This involves estimating operators defined on moving surfaces, therefore technically it is more complicated. We will use the results of Coifman, McIntosh and Meyer [4] and Coifman, David and Meyer [5] to estimate our operators. We point out here that for our operators, we don't really need such strong results. Our surfaces are more regular than those studied in [4], [5].

We divide this section into three parts: estimates, linear system, and the quasilinear system. Throughout the rest of this paper, we assume that $q$ is a constant $>1$.

Estimates. Let $s \geq 3 / 2+q$. For $t \in[0, T]$, let $\Sigma(t): \xi=\xi(\alpha, \beta, t),-\infty<\alpha, \beta<$ $\infty$, be a surface dividing $R^{3}$ into two unbounded, simply connected, lower and upper domains $\Omega(t)$ and $\Omega(t)^{c}$, with $\xi_{\alpha} \times \xi_{\beta}$ an outer normal of $\Omega(t)$. Assume that there are constants $C_{0}>0, \mu>0$, and vectors $e_{\alpha}, e_{\beta}$, such that

H1. $\left|\xi(\alpha, \beta, t)-\xi\left(\alpha^{\prime}, \beta^{\prime}, t\right)\right| \geq C_{0}\left(\left|\alpha-\alpha^{\prime}\right|+\left|\beta-\beta^{\prime}\right|\right)$, for $-\infty<\alpha, \beta<\infty, t \in$ $[0, T]$

H2. $\xi_{\alpha}-e_{\alpha}, \xi_{\beta}-e_{\beta} \in C^{1}\left([0, T], H^{s-1 / 2}\left(R^{2}, \mathcal{C}\left(V_{3}\right)\right)\right), e_{\alpha} \times e_{\beta}=e_{3}$ and $\xi_{t} \in$ $C\left([0, T], H^{s+1 / 2}\left(R^{2}, \mathcal{C}\left(V_{3}\right)\right)\right)$

H3. $\left|\xi_{\alpha} \times \xi_{\beta}\right| \geq \mu, \quad$ for $t \in[0, T]$.

In this part, we establish estimates that are necessary for obtaining an energy estimate and carrying out an iteration scheme for the linear system defined on $\Sigma(t)$, $t \in[0, T]$. We will not distinguish the notation of constants appearing in different contexts if they are determined by the same factors. For fixed $t, \mathcal{K}$ is the double layered potential defined on $\Sigma(t)$ w.r.t. $\Omega(t), \mathbf{n}=\frac{\xi_{\alpha} \times \xi_{\beta}}{\left|\xi_{\alpha} \times \xi_{\beta}\right|}, \nabla_{\mathbf{n}}$ is the normal derivative defined on $\Sigma(t)$, and $d S$ is the surface measure of $\Sigma(t)$. In the rest of this section, we use the same notation $u$ to indicate a function $u=u(\alpha, \beta)$ and the function $U(\xi, t)$ on $\Sigma(t)$, satisfying $U(\xi(\alpha, \beta, t), t)=u(\alpha, \beta)$.

Lemma 6.1 (Sobolev embedding [10]).

$$
H^{q}\left(R^{2}\right) \subset L_{p}\left(R^{2}\right) \quad \text { for } 2 \leq p \leq \infty .
$$

Lemma 6.2. Let $0 \leq \tau<1$, let $m$ be an integer $\geq 1$, and let $s \geq 1$. For $a, u \in$ $\mathfrak{S}\left(R^{2}\right)$, we have

a. $\left\|\left[a, \partial^{m}(1+|D|)^{\tau}\right] u\right\| \leq k_{0}\left(\|a\|_{m+\tau}\|u\|_{q}+\|u\|_{m+\tau-1}\|a\|_{q+1}\right)$.

b. $\left\|\left[a,(1+|D|)^{s}\right] u\right\| \leq k_{0}\left(\|a\|_{s}\|u\|_{q}+\|u\|_{s-1}\|a\|_{q+1}\right)$.

c. $\left\|\left[a,(1+|D|)^{\tau}\right] u\right\| \leq k_{0}\|a\|_{1+q}\|u\|$.

d. $\left\|\left[a,|D|^{1 / 2}\right]|D|^{1 / 2} u\right\| \leq k_{0}\|a\|_{1+q}\|u\|$.

where $k_{0}$ is a constant independent of $a$ and $u$.

Lemma 6.2 can be proved easily by using Fourier analysis. We omit the proof.

The following deep result was obtained by Coifman, McIntosh and Meyer [4] for $n=2$, and Coifman, David and Meyer [5] for general $n$.

Theorem 6.3 ([4], [5], see also [13]). Let $\theta: R^{k} \rightarrow R$ be even and $C^{\infty}$, and let $A: R^{n-1} \rightarrow R, B: R^{n-1} \rightarrow R^{k}$ be Lipschitz and

$$
K(z, x)=\frac{A(z)-A(x)}{|z-x|^{n}} \theta\left[\frac{B(z)-B(x)}{|z-x|}\right] .
$$


Then the operator

$$
T f(z)=p . v \cdot \int K(z, x) g(x) d x
$$

is bounded from $L_{2}\left(R^{n-1}\right)$ to $L_{2}\left(R^{n-1}\right)$, with

$$
\|T f\| \leq C\|f\|
$$

where $C=C(M, \theta, n)$, and $|\nabla A|_{\infty} \leq M,|\nabla B|_{\infty} \leq M$.

Lemma 6.4. Let $t \in[0, T]$ be fixed. For $u \in \mathfrak{S}\left(R^{2}\right), a \in H^{p_{1}}\left(R^{2}\right), b \in H^{p_{2}}\left(R^{2}\right)$, we have

a. $H_{\Sigma(t)}$ is bounded from $H^{r}\left(R^{2}\right)$ to $H^{r}\left(R^{2}\right)$, for $0 \leq r \leq s+1 / 2$, with $\left\|H_{\Sigma(t)} u\right\|_{r} \leq k_{r, 1}\|u\|_{r}$

b. $\left\|\left[a, H_{\Sigma(t)}\right] u\right\|_{r} \leq k_{r, 1}\|a\|_{\max \{r, 1+q\}}\|u\|_{r-1}$, for $1 \leq r \leq \min \left\{p_{1}, s+1 / 2\right\}$,

c. $\quad\left\|\left[a,\left[b, H_{\Sigma(t)}\right]\right] u\right\|_{r} \leq k_{r, 1}\|a\|_{\max \{r, 1+q\}}\|b\|_{\max \{r, 1+q\}}\|u\|_{r-2}$, for $2 \leq r \leq$ $\min \left\{p_{1}, p_{2}, s+1 / 2\right\}$

d. $\left( \pm I+\mathcal{K}^{*}\right)^{-1}$ and $( \pm I+\mathcal{K})^{-1}$ are bounded from $H^{r-1}\left(R^{2}\right)$ to $H^{r-1}\left(R^{2}\right)$, for $1 \leq r \leq s+1 / 2$, with

$$
\left\|\left( \pm I+\mathcal{K}^{*}\right)^{-1} u\right\|_{r-1} \leq k_{r, 2}\|u\|_{r-1} \quad \text { and } \quad\left\|( \pm I+\mathcal{K})^{-1} u\right\|_{r-1} \leq k_{r, 2}\|u\|_{r-1},
$$

where $k_{r, 1}=k_{r, 1}\left(C_{0}, \mu, M(r)\right), k_{r, 2}=k_{r, 2}\left(C_{0}, \mu, M(r), M_{1}\right)$ are constants,

$$
\left\|\xi_{\alpha}-e_{\alpha}\right\|_{\max \{r-1,1+q\}}+\left\|\xi_{\beta}-e_{\beta}\right\|_{\max \{r-1,1+q\}} \leq M(r), \quad\left\|\left( \pm I+\mathcal{K}^{*}\right)^{-1}\right\| \leq M_{1},
$$

$\left\|\left( \pm I+\mathcal{K}^{*}\right)^{-1}\right\|$ is the operator norm of $\left( \pm I+\mathcal{K}^{*}\right)^{-1}$ from $L_{2}\left(R^{2}\right)$ to $L_{2}\left(R^{2}\right)$.

Remark. If for some fixed $t$, we assume further that $\mathbf{n}(t)-e_{3}=\frac{\xi_{\alpha}(t) \times \xi_{\beta}(t)}{\left|\xi_{\alpha}(t) \times \xi_{\beta}(t)\right|}-e_{3} \in$ $H^{s}\left(R^{2}, C\left(V_{3}\right)\right)$, then $\left( \pm I+\mathcal{K}^{*}(t)\right)^{-1}$ is also bounded from $H^{s}\left(R^{2}\right)$ to $H^{s}\left(R^{2}\right)$, where $\mathcal{K}^{*}(t)$ is the adjoint of the double layered potential $\mathcal{K}(t)$ defined on $\Sigma(t)$.

Proof of $a$. We first prove $a$. for $r$ integers, where $r=0,1, \ldots,[s+1 / 2],[s+1 / 2]$ is the largest integer $\leq s+1 / 2$. We prove by induction.

When $r=0$, a. follows directly from Theorem 6.3 by taking $\theta$ even, $\theta \in C^{\infty}$, and $\theta(\eta)=\frac{1}{|\eta|^{3}}$, on $|\eta| \geq C_{0}, B(\alpha, \beta)=\xi(\alpha, \beta, t)$ and $A=A(\alpha, \beta)$ the components of $\xi(\alpha, \beta, t)$.

When $r=1$, since

$$
\partial H_{\Sigma(t)} u=\left[\partial, H_{\Sigma(t)}\right] u+H_{\Sigma(t)} \partial u
$$

and from (3.3),

$$
\begin{aligned}
& {\left[\partial, H_{\Sigma(t)}\right] u=p . v \cdot \iint K\left(\xi^{\prime}-\xi\right)\left(\left(\partial \xi-\partial^{\prime} \xi^{\prime}\right) \times\left(\xi_{\beta^{\prime}}^{\prime} u_{\alpha^{\prime}}^{\prime}-\xi_{\alpha^{\prime}}^{\prime} u_{\beta^{\prime}}^{\prime}\right)\right) d \alpha^{\prime} d \beta^{\prime}} \\
& \quad=p . v \cdot \iint K\left(\xi^{\prime}-\xi\right)\left(\xi_{\alpha^{\prime}}^{\prime} \times \xi_{\beta^{\prime}}^{\prime}\right) \frac{-\xi_{\alpha^{\prime}}^{\prime} \times \xi_{\beta^{\prime}}^{\prime}}{\left|\xi_{\alpha^{\prime}}^{\prime} \times \xi_{\beta^{\prime}}^{\prime}\right|^{2}}\left(\left(\partial \xi-\partial^{\prime} \xi^{\prime}\right) \times\left(\xi_{\beta^{\prime}}^{\prime} u_{\alpha^{\prime}}^{\prime}-\xi_{\alpha^{\prime}}^{\prime} u_{\beta^{\prime}}^{\prime}\right)\right) d \alpha^{\prime} d \beta^{\prime} \\
& \quad=\sum_{i}\left[a_{i}, H_{\Sigma(t)}\right] \mathfrak{D}_{i} u=\sum_{i}\left(a_{i} H_{\Sigma(t)} \mathfrak{D}_{i} u-H_{\Sigma(t)}\left(a_{i} \mathfrak{D}_{i} u\right)\right)
\end{aligned}
$$


where $a_{i}$ are components of $\partial \xi$, that is, $\partial \xi=\sum a_{i} e_{i}, \mathfrak{D}_{i} u=-\frac{\xi_{\alpha} \times \xi_{\beta}}{\left|\xi_{\alpha} \times \xi_{\beta}\right|^{2}} e_{i} \times$ $\left(\xi_{\beta} u_{\alpha}-\xi_{\alpha} u_{\beta}\right)$, we have

$$
\partial H_{\Sigma(t)} u=\sum_{i}\left(a_{i} H_{\Sigma(t)} \mathfrak{D}_{i} u-H_{\Sigma(t)}\left(a_{i} \mathfrak{D}_{i} u\right)\right)+H_{\Sigma(t)} \partial u,
$$

therefore

$$
\left\|H_{\Sigma(t)} u\right\|_{1} \leq k_{1,1}\|u\|_{1}
$$

where $k_{1,1}$ depends on $|\partial \xi|_{\infty}, C_{0}, \mu$.

When $r=2$, we have from (6.3)

$$
\partial^{2} H_{\Sigma(t)} u=\sum_{i}\left\{\partial\left(a_{i} H_{\Sigma(t)} \mathfrak{D}_{i} u\right)-\partial\left(H_{\Sigma(t)}\left(a_{i} \mathfrak{D}_{i} u\right)\right)\right\}+\partial H_{\Sigma(t)} \partial u,
$$

therefore $\left\|H_{\Sigma(t)} u\right\|_{2} \leq k_{2,1}\|u\|_{2}$, where $k_{2,1}$ depends on $\left|\partial^{2} \xi\right|_{\infty}+|\partial \xi|_{\infty}, C_{0}, \mu$. By interpolation, we have that $a$. holds for all $0 \leq r \leq 2$.

Now for $m \geq 3$, assume that $H_{\Sigma(t)}$ is bounded from $H^{m-1}\left(R^{2}\right)$ to $H^{m-1}\left(R^{2}\right)$, where $2 \leq m-1<[s+1 / 2]$. From (6.3),

$$
\partial^{m} H_{\Sigma(t)} u=\sum_{i}\left\{\partial^{m-1}\left(a_{i} H_{\Sigma(t)} \mathfrak{D}_{i} u\right)-\partial^{m-1}\left(H_{\Sigma(t)}\left(a_{i} \mathfrak{D}_{i} u\right)\right)\right\}+\partial^{m-1} H_{\Sigma(t)} \partial u .
$$

Therefore by the induction hypothesis and Lemma 6.2 a., we get

$$
\left\|\partial^{m} H_{\Sigma(t)} u\right\| \leq k_{m}^{\prime}\|u\|_{m}
$$

where $k_{m}^{\prime}$ is a constant depending on $\left\|a_{i}-c_{i}\right\|_{m^{\prime}}, C_{0}$, and $\mu ; a_{i}-c_{i}$ are components of $\xi_{\alpha}-e_{\alpha}$ or $\xi_{\beta}-e_{\beta}, m^{\prime}=\max \{m-1,1+q\}$. In other words, $k_{m}^{\prime}$ is a constant depending on $\left\|\xi_{\alpha}-e_{\alpha}\right\|_{m^{\prime}},\left\|\xi_{\beta}-e_{\beta}\right\|_{m^{\prime}}, C_{0}$, and $\mu$. Using the induction hypothesis again, we obtain that

$$
\left\|H_{\Sigma(t)} u\right\|_{m} \leq k_{m, 1}\|u\|_{m}
$$

where $k_{m, 1}=k_{m, 1}\left(M(m), C_{0}, \mu\right)$, and $\left\|\xi_{\alpha}-e_{\alpha}\right\|_{m^{\prime}}+\left\|\xi_{\beta}-e_{\beta}\right\|_{m^{\prime}} \leq M(m)$. This proves $a$. for $r$ integers, where $0 \leq r \leq[s+1 / 2]$, consequently for all $0 \leq r \leq[s+1 / 2]$ by interpolation.

Now let's prove $a$. for all $0 \leq r \leq s+1 / 2$. Let $0<\tau<1$. From (6.3), we also have that

$$
\begin{aligned}
\partial^{m}(1+|D|)^{\tau} H_{\Sigma(t)} u= & \sum_{i}\left\{\partial^{m-1}(1+|D|)^{\tau}\left(a_{i} H_{\Sigma(t)} \mathfrak{D}_{i} u\right)\right. \\
& \left.\quad-\partial^{m-1}(1+|D|)^{\tau}\left(H_{\Sigma(t)}\left(a_{i} \mathfrak{D}_{i} u\right)\right)\right\} \\
& +\partial^{m-1}(1+|D|)^{\tau} H_{\Sigma(t)} \partial u
\end{aligned}
$$

Since $a$. holds for $r=\tau$, we conclude from a similar induction argument that $a$. holds for $r=1+\tau, \ldots, l+\tau$, where $l=[s+1 / 2]$, if $0<\tau \leq s+1 / 2-[s+1 / 2]$, and $l=[s+1 / 2]-1$, if $s+1 / 2-[s+1 / 2]<\tau<1$. Therefore $a$. holds for all $0 \leq r \leq s+1 / 2$.

Proof of $b$. We only give a detailed proof of $b$. for $r$ integers. For noninteger $r$, the proof can be carried out using interpolation and induction, as we did for $a$.

Since

$$
\partial\left[a, H_{\Sigma(t)}\right] u=(\partial a) H_{\Sigma(t)} u+p . v . \iint\left(a-a^{\prime}\right) \partial K\left(\xi^{\prime}-\xi\right)\left(\xi_{\alpha^{\prime}}^{\prime} \times \xi_{\beta^{\prime}}^{\prime}\right) u^{\prime} d \alpha^{\prime} d \beta^{\prime},
$$


and p.v. $\iint\left(a-a^{\prime}\right) \partial K\left(\xi^{\prime}-\xi\right)\left(\xi_{\alpha^{\prime}}^{\prime} \times \xi_{\beta^{\prime}}^{\prime}\right) u^{\prime} d \alpha^{\prime} d \beta^{\prime}$ has the type of kernel as in Theorem 6.3 , and since $\left[a, H_{\Sigma(t)}\right]$ is bounded from $L_{2}\left(R^{2}\right)$ to $L_{2}\left(R^{2}\right)$, we know that $b$. holds for $r=1$.

Assume that $b$. holds for $r=m-1$, where $1 \leq m-1<\min \left\{p_{1}, s+1 / 2\right\}$. Since

$$
\begin{aligned}
\partial^{m}\left[a, H_{\Sigma(t)}\right] u & =\left[\partial^{m}, a\right] H_{\Sigma(t)} u+\left[a, \partial^{m} H_{\Sigma(t)}\right] u \\
& =\left[\partial^{m}, a\right] H_{\Sigma(t)} u+\left[a, \partial^{m-1}\left[\partial, H_{\Sigma(t)}\right]\right] u \\
& +\left[a, \partial^{m-1} H_{\Sigma(t)}\right] \partial u-\partial^{m-1} H_{\Sigma(t)}((\partial a) u)
\end{aligned}
$$

and from (6.2),

$$
\begin{aligned}
{\left[a, \partial^{m-1}\left[\partial, H_{\Sigma(t)}\right]\right] u } & =a \partial^{m-1}\left[\partial, H_{\Sigma(t)}\right] u-\partial^{m-1}\left[\partial, H_{\Sigma(t)}\right](a u) \\
& =a \sum_{i} \partial^{m-1}\left[a_{i}, H_{\Sigma(t)}\right] \mathfrak{D}_{i} u-\sum_{i} \partial^{m-1}\left[a_{i}, H_{\Sigma(t)}\right] \mathfrak{D}_{i}(a u)
\end{aligned}
$$

where $a_{i}-c_{i} \in H^{s-1 / 2}\left(R^{2}\right)$ are components of $\xi_{\alpha}-e_{\alpha}$ or $\xi_{\beta}-e_{\beta}$, and since

$$
\left[a, \partial^{m-1} H_{\Sigma(t)}\right] \partial u=\partial^{m-1}\left[a, H_{\Sigma(t)}\right] \partial u-\left[\partial^{m-1}, a\right] H_{\Sigma(t)} \partial u,
$$

we have first by taking $m=2$ that $b$. holds for $r=2$, therefore for all $1 \leq r \leq 2$ by interpolation. For general $m$, we get from the induction hypothesis, Lemma 6.2 a. and Lemma 6.4 a. that

$$
\left\|\partial^{m}\left[a, H_{\Sigma(t)}\right] u\right\| \leq k_{m}^{\prime \prime}\|a\|_{\max \{1+q, m\}}\|u\|_{m-1},
$$

where $k_{m}^{\prime \prime}$ depends on $\left\|\xi_{\alpha}-e_{\alpha}\right\|_{m^{\prime}},\left\|\xi_{\beta}-e_{\beta}\right\|_{m^{\prime}}, C_{0}$ and $\mu, m^{\prime}=\max \{1+q, m-1\}$. Using the induction hypothesis again, we obtain

$$
\left\|\left[a, H_{\Sigma(t)}\right] u\right\|_{m} \leq k_{m, 1}\|u\|_{m-1},
$$

where $k_{m, 1}=k_{m, 1}\left(M(m), C_{0}, \mu\right)$, and $\left\|\xi_{\alpha}-e_{\alpha}\right\|_{m^{\prime}}+\left\|\xi_{\beta}-e_{\beta}\right\|_{m^{\prime}} \leq M(m)$. This proves $b$. for integer $r$.

Proof of $c$. and $d$. $c$. $d$. are proved in the same way as we did for $a$. and $b$. That is, we first prove by induction for $r$ integers, then by interpolation and induction for all $2 \leq r \leq \min \left\{p_{1}, p_{2}, s+1 / 2\right\}$ for $c$. and all $1 \leq r \leq s+1 / 2$ for $d$. The induction argument for $c$. is carried out using identities

$$
\begin{gathered}
\partial^{m}\left[a,\left[b, H_{\Sigma(t)}\right]\right] u=\left[\partial^{m}, a\right]\left[b, H_{\Sigma(t)}\right] u+\left[a, \partial^{m-1}\left[\partial,\left[b, H_{\Sigma(t)}\right]\right]\right] u \\
+\left[a, \partial^{m-1}\left[b, H_{\Sigma(t)}\right]\right] \partial u-\partial^{m-1}\left[b, H_{\Sigma(t)}\right]((\partial a) u), \\
{\left[\partial,\left[b, H_{\Sigma(t)}\right]\right] u=\left[\partial b, H_{\Sigma(t)}\right] u+\left[b,\left[\partial, H_{\Sigma(t)}\right]\right] u} \\
=\left[\partial b, H_{\Sigma(t)}\right] u+\sum_{i}\left[b,\left[a_{i}, H_{\Sigma(t)}\right] \mathfrak{D}_{i}\right] u \\
=\left[\partial b, H_{\Sigma(t)}\right] u+\sum_{i}\left\{\left[b,\left[a_{i}, H_{\Sigma(t)}\right]\right] \mathfrak{D}_{i} u-\left[a_{i}, H_{\Sigma(t)}\right]\left[\mathfrak{D}_{i}, b\right] u\right\}
\end{gathered}
$$

and

$$
\left[a, \partial^{m-1}\left[b, H_{\Sigma(t)}\right]\right] \partial u=\partial^{m-1}\left[a,\left[b, H_{\Sigma(t)}\right]\right] \partial u-\left[\partial^{m-1}, a\right]\left[b, H_{\Sigma(t)}\right] \partial u
$$


Theorem 6.3 and Lemma $6.4 \mathrm{~b}$. The induction argument for $d$. with regard to $\left( \pm I+\mathcal{K}^{*}\right)^{-1}$ is carried out using Theorem 2.4, identities

$$
\begin{aligned}
\partial^{m}\left( \pm I+\mathcal{K}^{*}\right)^{-1} & =\partial^{m-1}\left[\partial,\left( \pm I+\mathcal{K}^{*}\right)^{-1}\right]+\partial^{m-1}\left( \pm I+\mathcal{K}^{*}\right)^{-1} \partial \\
& =-\partial^{m-1}\left( \pm I+\mathcal{K}^{*}\right)^{-1}\left[\partial, \mathcal{K}^{*}\right]\left( \pm I+\mathcal{K}^{*}\right)^{-1}+\partial^{m-1}\left( \pm I+\mathcal{K}^{*}\right)^{-1} \partial
\end{aligned}
$$

and (6.2), and Lemma $6.4 a$. b. It is similar for $( \pm I+\mathcal{K})^{-1}$.

As an immediate consequence of Lemma 6.4, (3.13) and (3.14), we have the following

Corollary 6.5. Let $u \in \mathfrak{S}\left(R^{2}\right)$. Then for $1 \leq r \leq s+1 / 2$,

$$
k_{r, 2}^{-1}\left(\left\|\partial_{\alpha} u\right\|_{r-1}+\left\|\partial_{\beta} u\right\|_{r-1}\right) \leq\left\|\nabla_{\mathbf{n}} u\right\|_{r-1} \leq k_{r, 2}\left(\left\|\partial_{\alpha} u\right\|_{r-1}+\left\|\partial_{\beta} u\right\|_{r-1}\right)
$$

where $k_{r, 2}=k_{r, 2}\left(C_{0}, \mu, M(r), M_{1}\right) \geq 1, M(r)$ and $M_{1}$ are as in Lemma 6.4.

Lemma 6.6. Let $a \in H^{1+q}\left(R^{2}\right)$. For $u, w \in \mathfrak{S}\left(R^{2}\right)$, we have

$a$.

$$
\begin{aligned}
& \left|\int_{\Sigma(t)}\left(a w \nabla_{\mathbf{n}} u+a u \nabla_{\mathbf{n}} w\right) d S\right| \\
& \quad \leq \kappa_{0}\|a\|_{1+q}\left(\|u\|+\left(\int_{\Sigma(t)} u \nabla_{\mathbf{n}} u d S\right)^{\frac{1}{2}}\right)\left(\|w\|+\left(\int_{\Sigma(t)} w \nabla_{\mathbf{n}} w d S\right)^{\frac{1}{2}}\right),
\end{aligned}
$$

b. $\left|\int_{\Sigma(t)} w \nabla_{\mathbf{n}} u d S\right| \leq\left(\int_{\Sigma(t)} u \nabla_{\mathbf{n}} u d S\right)^{\frac{1}{2}}\left(\int_{\Sigma(t)} w \nabla_{\mathbf{n}} w d S\right)^{\frac{1}{2}}$,

c. $\left|\int_{\Sigma(t)} w\left[a, \nabla_{\mathbf{n}}\right] u d S\right| \leq \kappa_{0}\|a\|_{1+q}\|u\|\left(\|w\|+\left(\int_{\Sigma(t)} w \nabla_{\mathbf{n}} w d S\right)^{\frac{1}{2}}\right)$

where $\kappa_{0}=\kappa_{0}\left(C_{0}, \mu, M, M_{1}\right)$, and $\left\|\xi_{\alpha}-e_{\alpha}\right\|_{1+q}+\left\|\xi_{\beta}-e_{\beta}\right\|_{1+q} \leq M, M_{1}$ is as in Lemma 6.4.

Remark. If $a-1 \in H^{1+q}\left(R^{2}\right)$, Lemma 6.6 holds with $\|a\|_{1+q}$ in $a$. and $c$. replaced by $\|a-1\|_{1+q}+1$.

Proof. We prove Lemma 6.6 by Green's identity.

Let $a^{\hbar}, u^{\hbar}$, and $w^{\hbar}$ be the harmonic extension, given by Theorem 2.5, of $a, u$, and $w$ on $\Omega(t)$ respectively. By definition: $\nabla_{\mathbf{n}} u=\frac{\partial u^{\hbar}}{\partial \mathbf{n}}$ and Green's second identity, we have

$$
\begin{aligned}
\int_{\Sigma(t)}\left(a \nabla_{\mathbf{n}} u w+a u \nabla_{\mathbf{n}} w\right) d S=\int_{\Sigma(t)} a \frac{\partial\left(u^{\hbar} w^{\hbar}\right)}{\partial \mathbf{n}} d S \\
=\int_{\Sigma(t)} \frac{\partial a^{\hbar}}{\partial \mathbf{n}} u w d S+2 \int_{\Omega(t)} a^{\hbar} \nabla u^{\hbar} \cdot \nabla w^{\hbar} d V
\end{aligned}
$$

Since

$$
\left|\int_{\Sigma(t)} \frac{\partial a^{\hbar}}{\partial \mathbf{n}} u w d S\right|=\left|\int_{\Sigma(t)}\left(\nabla_{\mathbf{n}} a\right) u w d S\right| \leq\left|\nabla_{\mathbf{n}} a\right|_{\infty} \int_{\Sigma(t)}|u w| d S
$$

and

$$
\begin{aligned}
2\left|\int_{\Omega(t)} a^{\hbar} \nabla u^{\hbar} \cdot \nabla w^{\hbar} d V\right| & \leq 2\left|a^{\hbar}\right|_{\infty}\left(\int_{\Omega(t)}\left|\nabla u^{\hbar}\right|^{2} d V\right)^{1 / 2}\left(\int_{\Omega(t)}\left|\nabla w^{\hbar}\right|^{2} d V\right)^{1 / 2} \\
& \leq 2|a|_{\infty}\left(\int_{\Sigma(t)} u \nabla_{\mathbf{n}} u d S\right)^{1 / 2}\left(\int_{\Sigma(t)} w \nabla_{\mathbf{n}} w d S\right)^{1 / 2}
\end{aligned}
$$


where in the last inequality we used the maximal principle: $\left|a^{\hbar}\right|_{\infty} \leq|a|_{\infty}$ and Green's identity, therefore

$$
\begin{aligned}
& \left|\int_{\Sigma(t)}\left(a \nabla_{\mathbf{n}} u w+a u \nabla_{\mathbf{n}} w\right) d S\right| \leq\left|\nabla_{\mathbf{n}} a\right|_{\infty} \int_{\Sigma(t)}|u w| d S \\
& \quad+2|a|_{\infty}\left(\int_{\Sigma(t)} u \nabla_{\mathbf{n}} u d S\right)^{1 / 2}\left(\int_{\Sigma(t)} w \nabla_{\mathbf{n}} w d S\right)^{1 / 2} .
\end{aligned}
$$

Applying Lemma 6.1 and Corollary 6.5 to (6.10), we obtain $a$.

$b$. is a consequence of (6.10) by taking $a=1$ :

$$
\begin{aligned}
\left|2 \int_{\Sigma(t)} \nabla_{\mathbf{n}} u w d S\right| & =\left|\int_{\Sigma(t)}\left(\nabla_{\mathbf{n}} u w+u \nabla_{\mathbf{n}} w\right) d S\right| \\
& \leq 2\left(\int_{\Sigma(t)} u \nabla_{\mathbf{n}} u d S\right)^{1 / 2}\left(\int_{\Sigma(t)} w \nabla_{\mathbf{n}} w d S\right)^{1 / 2}
\end{aligned}
$$

Now let's prove $c$. From Green's identity and by definition, we have

$$
\begin{aligned}
\int_{\Sigma(t)} & {\left[a, \nabla_{\mathbf{n}}\right] u w d S=\int_{\Sigma(t)} a w \frac{\partial u^{\hbar}}{\partial \mathbf{n}} d S-\int_{\Sigma(t)} \nabla_{\mathbf{n}}(a u) w d S } \\
& =\int_{\Sigma(t)} \frac{\partial\left(a^{\hbar} w^{\hbar}\right)}{\partial \mathbf{n}} u^{\hbar} d S-2 \int_{\Omega(t)} u^{\hbar} \nabla a^{\hbar} \cdot \nabla w^{\hbar} d V-\int_{\Sigma(t)} a u \frac{\partial w^{\hbar}}{\partial \mathbf{n}} d S \\
& =\int_{\Sigma(t)} \frac{\partial a^{\hbar}}{\partial \mathbf{n}} u w d S-2 \int_{\Omega(t)} u^{\hbar} \nabla a^{\hbar} \cdot \nabla w^{\hbar} d V,
\end{aligned}
$$

where

$$
\left|2 \int_{\Omega(t)} u^{\hbar} \nabla a^{\hbar} \cdot \nabla w^{\hbar} d V\right| \leq\left(2 \int_{\Omega(t)}\left(u^{\hbar}\right)^{2}\left|\nabla a^{\hbar}\right|^{2} d V\right)^{1 / 2}\left(2 \int_{\Omega(t)}\left|\nabla w^{\hbar}\right|^{2} d V\right)^{1 / 2} .
$$

Assume that $\tilde{a}$ and $\tilde{u}$ are the solutions of

$$
\left\{\begin{array} { r l } 
{ \Delta \tilde { a } = 2 | \nabla a ^ { \hbar } | ^ { 2 } } & { \text { on } \Omega ( t ) , } \\
{ \tilde { a } = 0 } & { \text { on } \Sigma ( t ) , }
\end{array} \quad \text { and } \quad \left\{\begin{array}{rlrl}
\Delta \tilde{u} & =0 & & \text { on } \Omega(t), \\
\tilde{u} & =u^{2} & \text { on } \Sigma(t),
\end{array}\right.\right.
$$

given by Theorem 2.5. (Notice that $\tilde{a}-\left(a^{\hbar}\right)^{2}$ is harmonic on $\Omega(t)$.) Since $\Delta\left(u^{\hbar}\right)^{2}=$ $2\left|\nabla u^{\hbar}\right|^{2} \geq 0$ and $\left(u^{\hbar}\right)^{2}=\tilde{u}$ on $\Sigma(t)$, we have from the maximal principle that $\left(u^{\hbar}\right)^{2} \leq \tilde{u}$ on $\Omega(t)$. Therefore

$$
2 \int_{\Omega(t)}\left(u^{\hbar}\right)^{2}\left|\nabla a^{\hbar}\right|^{2} d V \leq 2 \int_{\Omega(t)} \tilde{u}\left|\nabla a^{\hbar}\right|^{2} d V=\int_{\Sigma(t)} u^{2} \frac{\partial \tilde{a}}{\partial \mathbf{n}} d S .
$$

Now from Theorem 2.5, we have that

$$
\tilde{a}(\eta)=\left(a^{\hbar}\right)^{2}(\eta)+\int_{\Sigma(t)} K\left(\xi^{\prime}-\eta\right) \cdot \mathbf{n}\left(\xi^{\prime}\right)(I+\mathcal{K})^{-1}\left(a^{2}\right)\left(\xi^{\prime}\right) d S\left(\xi^{\prime}\right), \quad \eta \in \Omega(t),
$$

so

$$
\left|\frac{\partial \tilde{a}}{\partial \mathbf{n}}\right|_{\infty} \leq k_{0}\left\|\frac{\partial \tilde{a}}{\partial \mathbf{n}}\right\|_{q} \leq \kappa_{0}^{\prime}\|a\|_{1+q}^{2}
$$


Consequently,

$$
\begin{aligned}
& \left|\int_{\Sigma(t)}\left[a, \nabla_{\mathbf{n}}\right] u w d S\right| \\
& \quad \leq \kappa_{0}^{\prime}\|a\|_{1+q}\left(\int_{\Sigma(t)}|u w| d S+\left(\int_{\Sigma(t)} u^{2} d S\right)^{1 / 2}\left(\int_{\Sigma(t)} w \nabla_{\mathbf{n}} w d S\right)^{1 / 2}\right) \\
& \quad \leq \kappa_{0}\|a\|_{1+q}\|u\|\left(\|w\|+\left(\int_{\Sigma(t)} w \nabla_{\mathbf{n}} w d S\right)^{\frac{1}{2}}\right)
\end{aligned}
$$

where $\kappa_{0}=\kappa_{0}\left(C_{0}, \mu, M, M_{1}\right)$, and $\left\|\xi_{\alpha}-e_{\alpha}\right\|_{1+q}+\left\|\xi_{\beta}-e_{\beta}\right\|_{1+q} \leq M, M_{1}$ is as in Lemma 6.4. This proves $c$.

Remark. Green's identity is justified here since $a^{\hbar}$, $u^{\hbar}$, and $w^{\hbar}$ have enough integrability and $u^{\hbar}, w^{\hbar}$ have enough decay at infinity; see Theorem 2.5 and it's remark.

Lemma 6.7. For $u \in \mathfrak{S}\left(R^{2}\right)$, we have

$$
\kappa_{0}^{-1}\|u\|_{1 / 2}^{2} \leq\|u\|^{2}+\int_{\Sigma(t)} u \nabla_{\mathbf{n}} u d S \leq \kappa_{0}\|u\|_{1 / 2}^{2}
$$

where $\kappa_{0}=\kappa_{0}\left(C_{0}, \mu, M, M_{1}\right) \geq 1, M, M_{1}$ are as in Lemma 6.6.

Proof. We first prove the right hand side inequality of (6.13). Let $\left(R_{1}, R_{2}\right)=$ $\left(\partial_{\alpha}|D|^{-1}, \partial_{\beta}|D|^{-1}\right)$ be the Riesz transforms on $R^{2}$,

$$
\mathcal{R} w=\left(I+\mathcal{K}^{*}\right)^{-1}\left\{p . v . \iint\left(\mathbf{n}(\xi) \times K\left(\xi^{\prime}-\xi\right)\right) \cdot\left(\xi_{\beta^{\prime}}^{\prime} R_{1} w-\xi_{\alpha^{\prime}}^{\prime} R_{2} w\right) d \alpha^{\prime} d \beta^{\prime}\right\},
$$

and let $\mathcal{R}^{*}$ be the adjoint of $\mathcal{R}$ on $L^{2}(\Sigma(t), d S)$. From (3.13) we know $\nabla_{\mathbf{n}} u=\mathcal{R}|D| u$. Therefore

$$
\begin{aligned}
\int_{\Sigma(t)} u \nabla_{\mathbf{n}} u d S & =\int_{\Sigma(t)} \mathcal{R}^{*}(u)|D| u d S=\iint|D|^{1 / 2}\left(\left|\xi_{\alpha} \times \xi_{\beta}\right| \mathcal{R}^{*}(u)\right)|D|^{1 / 2} u d \alpha d \beta \\
& \leq\left(\iint\left\{|D|^{1 / 2}\left(\left|\xi_{\alpha} \times \xi_{\beta}\right| \mathcal{R}^{*}(u)\right)\right\}^{2} d \alpha d \beta\right)^{1 / 2}\left(\iint\left\{|D|^{1 / 2} u\right\}^{2} d \alpha d \beta\right)^{1 / 2} \\
& \leq \kappa_{0}^{\prime}\|u\|_{1 / 2}^{2},
\end{aligned}
$$

where the last inequality follows from Lemma 6.4 and Lemma 6.2 , and $\kappa_{0}^{\prime}=$ $\kappa_{0}^{\prime}\left(C_{0}, \mu, M, M_{1}\right),\left\|\xi_{\alpha}-e_{\alpha}\right\|_{1+q}+\left\|\xi_{\beta}-e_{\beta}\right\|_{1+q} \leq M$, and $M_{1}$ is as in Lemma 6.4. This proves the right hand side inequality of (6.13).

The left hand side inequality of (6.13) is proved similarly. We know from (3.14) that

$$
\partial u=-\partial \xi \cdot\left(I+H_{\Sigma(t)}\right)\left(\mathbf{n}\left(-I+\mathcal{K}^{*}\right)^{-1} \nabla_{\mathbf{n}} u\right) .
$$

Since $|D|=-R_{1} \partial_{\alpha}-R_{2} \partial_{\beta}$, we can also write $|D| u=\tilde{\mathcal{R}} \nabla_{\mathbf{n}} u$, where

$$
\begin{aligned}
\tilde{\mathcal{R}} w=R_{1}\left\{\xi_{\alpha} \cdot\left(I+H_{\Sigma(t)}\right)\left(\mathbf{n}\left(-I+\mathcal{K}^{*}\right)^{-1} w\right)\right\} & \\
& +R_{2}\left\{\xi_{\beta} \cdot\left(I+H_{\Sigma(t)}\right)\left(\mathbf{n}\left(-I+\mathcal{K}^{*}\right)^{-1} w\right)\right\} .
\end{aligned}
$$


Let $\tilde{\mathcal{R}}^{*}$ be the adjoint of $\tilde{\mathcal{R}}$ on $L^{2}(\Sigma(t), d S)$. Therefore

$$
\iint u|D| u d \alpha d \beta=\int_{\Sigma(t)} \frac{1}{\left|\xi_{\alpha} \times \xi_{\beta}\right|} u \tilde{\mathcal{R}} \nabla_{\mathbf{n}} u d S=\int_{\Sigma(t)} \tilde{\mathcal{R}}^{*}\left\{\frac{1}{\left|\xi_{\alpha} \times \xi_{\beta}\right|} u\right\} \nabla_{\mathbf{n}} u d S .
$$

Applying Lemma 6.6 , we get

$$
\begin{aligned}
& \int_{\Sigma(t)} \tilde{\mathcal{R}}^{*}\left\{\frac{u}{\left|\xi_{\alpha} \times \xi_{\beta}\right|}\right\} \nabla_{\mathbf{n}} u d S \\
& \quad \leq\left(\int_{\Sigma(t)} u \nabla_{\mathbf{n}} u d S\right)^{1 / 2}\left(\int_{\Sigma(t)} \tilde{\mathcal{R}}^{*}\left\{\frac{u}{\left|\xi_{\alpha} \times \xi_{\beta}\right|}\right\} \nabla_{\mathbf{n}} \tilde{\mathcal{R}}^{*}\left\{\frac{u}{\left|\xi_{\alpha} \times \xi_{\beta}\right|}\right\} d S\right)^{1 / 2},
\end{aligned}
$$

where

$$
\begin{aligned}
\int_{\Sigma(t)} & \tilde{\mathcal{R}}^{*}\left\{\frac{u}{\left|\xi_{\alpha} \times \xi_{\beta}\right|}\right\} \nabla_{\mathbf{n}} \tilde{\mathcal{R}}^{*}\left\{\frac{u}{\left|\xi_{\alpha} \times \xi_{\beta}\right|}\right\} d S=\iint u|D|\left(\tilde{\mathcal{R}}^{*}\left\{\frac{u}{\left|\xi_{\alpha} \times \xi_{\beta}\right|}\right\}\right) d \alpha d \beta \\
\leq & \|u\|_{1 / 2}\left(\iint\left(|D|^{1 / 2}\left(\tilde{\mathcal{R}}^{*}\left\{\frac{u}{\left|\xi_{\alpha} \times \xi_{\beta}\right|}\right\}\right)\right)^{2} d \alpha d \beta\right)^{1 / 2} \\
\leq & \kappa_{0}^{\prime}\|u\|_{1 / 2}^{2} .
\end{aligned}
$$

Here we used Lemma 6.4 and Lemma 6.2 again in the last inequality. Therefore

$$
\iint u|D| u d \alpha d \beta \leq \sqrt{\kappa_{0}^{\prime}}\left(\int_{\Sigma(t)} u \nabla_{\mathbf{n}} u d S\right)^{1 / 2}\|u\|_{1 / 2}
$$

and consequently

$$
\|u\|_{1 / 2}^{2} \leq \kappa_{0}\left(\|u\|^{2}+\int_{\Sigma(t)} u \nabla_{\mathbf{n}} u d S\right)
$$

This proves the left hand side inequality of (6.13).

Using Lemmas 3.1, 3.2 and a similar argument as in Lemma 6.7, we also have the following

Lemma 6.8. Let $a-1 \in H^{1+q}\left(R^{2}\right)$. For $u \in \mathfrak{S}\left(R^{2}\right)$, we have

$$
\left|\iint a u\left[\partial_{t}, \nabla_{\mathbf{n}}\right] u d \alpha d \beta\right| \leq \kappa_{0}\left(\|a-1\|_{1+q}+1\right)\left\|\xi_{t}\right\|_{2+q}\|u\|_{1 / 2}^{2}
$$

where $\kappa_{0}=\kappa_{0}\left(C_{0}, \mu, M, M_{1}\right)$, and $M$ and $M_{1}$ are as in Lemma 6.6.

Let

$$
G=\mathfrak{a} \nabla_{\mathbf{n}}+\lambda, \quad a=\frac{\mathfrak{a}}{\left|\xi_{\alpha} \times \xi_{\beta}\right|},
$$

where $\mathfrak{a}-1 \in H^{1+q}\left(R^{2}\right)$ and $\lambda$ is a constant. Also let $(u, v)=\iint u v d \alpha d \beta$.

Lemma 6.9. Let $\mathfrak{a}-1 \in H^{1+q}\left(R^{2}\right)$. Assume that there is a constant $c_{0}>0$, such that $\mathfrak{a} \geq c_{0}$. Assume $\lambda \geq\left|\nabla_{\mathbf{n}} a\right|_{\infty}\left|\xi_{\alpha} \times \xi_{\beta}\right|_{\infty}+\frac{c_{0}}{\left|\xi_{\alpha} \times \xi_{\beta}\right|_{\infty}}$. Then for $u \in \mathfrak{S}\left(R^{2}\right)$,

$$
\kappa_{1}^{-1}\|u\|_{1 / 2}^{2} \leq(G u, u) \leq \kappa_{1}\|u\|_{1 / 2}^{2}
$$

where $\kappa_{1} \geq \max \left\{2 \lambda+\kappa_{0}|a|_{\infty}, \frac{\left|\xi_{\alpha} \times \xi_{\beta}\right|_{\infty}}{c_{0}} \kappa_{0}\right\}, \kappa_{0}$ is the constant in Lemma 6.\%. 
Proof. Let $a^{\hbar}-1, u^{\hbar}$ be the harmonic extensions of $a-1$ and $u$ on $\Omega(t)$, given by Theorem 2.5. From Green's second identity, we have that

$$
\int_{\Sigma(t)} a u \nabla_{\mathbf{n}} u d S=\frac{1}{2} \int_{\Sigma(t)} a^{\hbar} \frac{\partial\left(u^{\hbar}\right)^{2}}{\partial \mathbf{n}} d S=\frac{1}{2} \int_{\Sigma(t)} \frac{\partial a^{\hbar}}{\partial \mathbf{n}} u^{2} d S+\int_{\Omega(t)} a^{\hbar}\left|\nabla u^{\hbar}\right|^{2} d V,
$$

therefore

$$
\begin{aligned}
\int_{\Sigma(t)} a u \nabla_{\mathbf{n}} u d S & \geq-\left|\nabla_{\mathbf{n}} a\right| \xi_{\alpha} \times \xi_{\beta}\left\|_{\infty}\right\| u \|^{2}+\frac{c_{0}}{\left|\xi_{\alpha} \times \xi_{\beta}\right|_{\infty}} \int_{\Omega(t)}\left|\nabla u^{\hbar}\right|^{2} d V \\
& \geq-\left|\nabla_{\mathbf{n}} a\right|_{\infty}\left|\xi_{\alpha} \times \xi_{\beta}\right|_{\infty}\|u\|^{2}+\frac{c_{0}}{\left|\xi_{\alpha} \times \xi_{\beta}\right|_{\infty}} \int_{\Sigma(t)} u \nabla_{\mathbf{n}} u d S \\
& \geq-\left|\nabla_{\mathbf{n}} a\right|_{\infty}\left|\xi_{\alpha} \times \xi_{\beta}\right|_{\infty}\|u\|^{2}+\frac{c_{0}}{\left|\xi_{\alpha} \times \xi_{\beta}\right|_{\infty}}\left(\kappa_{0}^{-1}\|u\|_{1 / 2}^{2}-\|u\|^{2}\right),
\end{aligned}
$$

where in the first inequality we used the maximal principle that $a^{\hbar} \geq \min _{\Sigma(t)} a \geq$ $\frac{c_{0}}{\left|\xi_{\alpha} \times \xi_{\beta}\right|_{\infty}}$, and in the last inequality we used Lemma 6.7. Consequently,

$$
(G u, u)=\int_{\Sigma(t)} a u \nabla_{\mathbf{n}} u d S+\lambda\|u\|^{2} \geq \frac{c_{0}}{\left|\xi_{\alpha} \times \xi_{\beta}\right|_{\infty}} \kappa_{0}^{-1}\|u\|_{1 / 2}^{2} .
$$

On the other hand, we have from (6.19) that

$$
\begin{aligned}
(G u, u) & =\int_{\Sigma(t)} a u \nabla_{\mathbf{n}} u d S+\lambda\|u\|^{2} \\
& \leq\left|\nabla_{\mathbf{n}} a\right| \xi_{\alpha} \times \xi_{\beta}\left\|_{\infty}\right\| u\left\|^{2}+|a|_{\infty} \int_{\Omega(t)}\left|\nabla u^{\hbar}\right|^{2} d V+\lambda\right\| u \|^{2} \\
& \leq\left|\nabla_{\mathbf{n}} a\right|_{\infty}\left|\xi_{\alpha} \times \xi_{\beta}\right|_{\infty}\|u\|^{2}+|a|_{\infty} \int_{\Sigma(t)} u \nabla_{\mathbf{n}} u d S+\lambda\|u\|^{2} \\
& \leq\left(\left|\nabla_{\mathbf{n}} a\right|_{\infty}\left|\xi_{\alpha} \times \xi_{\beta}\right|_{\infty}+\lambda\right)\|u\|^{2}+\kappa_{0}|a|_{\infty}\|u\|_{1 / 2}^{2} \\
& \leq\left(2 \lambda+\kappa_{0}|a|_{\infty}\right)\|u\|_{1 / 2}^{2} .
\end{aligned}
$$

Taking $\kappa_{1} \geq \max \left\{2 \lambda+\kappa_{0}|a|_{\infty}, \frac{\left|\xi_{\alpha} \times \xi_{\beta}\right|_{\infty}}{c_{0}} \kappa_{0}\right\}$, we get (6.18).

For $t \in[0, T]$ and $0 \leq \vartheta \leq 1$, let

$$
\begin{aligned}
& \Sigma_{1}(t): \xi_{1}=\xi_{1}(\alpha, \beta, t), \quad \Sigma_{2}(t): \xi_{2}=\xi_{2}(\alpha, \beta, t), \\
& \Sigma(\vartheta ; t): \xi=\vartheta \xi_{1}(\alpha, \beta, t)+(1-\vartheta) \xi_{2}(\alpha, \beta, t) .
\end{aligned}
$$

Assume that $\Sigma(\vartheta ; t)$ satisfies H1 and H3 for all $t \in[0, T]$ and $0 \leq \vartheta \leq 1$; and $\Sigma_{1}(t)$, $\Sigma_{2}(t)$ satisfy $\mathrm{H} 1, \mathrm{H} 2^{\prime}, \mathrm{H} 3$, where

$\mathrm{H} 2^{\prime}: \xi_{\alpha}-e_{\alpha}, \xi_{\beta}-e_{\beta} \in C\left([0, T], H^{s-1 / 2}\left(R^{2}, \mathcal{C}\left(V_{3}\right)\right)\right), e_{\alpha} \times e_{\beta}=e_{3}$ and $\xi_{t} \in$ $L^{\infty}\left([0, T], H^{s+1 / 2}\left(R^{2}, \mathcal{C}\left(V_{3}\right)\right)\right)$.

Lemma 6.10. Let $t \in[0, T]$ be fixed. Then for $u \in \mathfrak{S}\left(R^{2}\right), a \in H^{p_{1}}\left(R^{2}\right), b \in$ $H^{p_{2}}\left(R^{2}\right)$, we have

a. $\left\|\left(H_{\Sigma_{1}(t)}-H_{\Sigma_{2}(t)}\right) u\right\|_{r} \leq k_{r, 1}\left\|\xi_{1}(t)-\xi_{2}(t)\right\|_{r}\|u\|_{r}$, for $2+q \leq r \leq s+1 / 2$,

b. $\left\|\left[a, H_{\Sigma_{1}(t)}-H_{\Sigma_{2}(t)}\right] u\right\|_{r} \leq k_{r, 1}\|a\|_{r}\left\|\xi_{1}(t)-\xi_{2}(t)\right\|_{r}\|u\|_{r-1}$, for $2+q \leq r \leq$ $\min \left\{p_{1}, s+1 / 2\right\}$, 
c. $\left\|\left[a,\left[b, H_{\Sigma_{1}(t)}-H_{\Sigma_{2}(t)}\right]\right] u\right\|_{r} \leq k_{r, 1}\|a\|_{r}\|b\|_{r}\left\|\xi_{1}(t)-\xi_{2}(t)\right\|_{r}\|u\|_{r-2}$, for $2+q \leq$ $r \leq \min \left\{p_{1}, p_{2}, s+1 / 2\right\}$,

where $k_{r, 1}=k_{r, 1}\left(C_{0}, \mu, M(r)\right)$, and $\left\|\xi_{i, \alpha}-e_{\alpha}\right\|_{r-1}+\left\|\xi_{i, \beta}-e_{\beta}\right\|_{r-1} \leq M(r), i=1,2$.

Proof. We prove $a$. only. $b$., $c$. can be obtained similarly. Actually, the proof of $c$. involves operators of type $\left[a,\left[b,\left[c, H_{\Sigma}\right]\right]\right]$. An estimate of such operators can be obtained similar to that in Lemma $6.4 \mathrm{~b}$. We leave $c$. to the readers.

We know that $\partial_{\vartheta} \xi=\xi_{1}-\xi_{2}$. Since

$$
\left(H_{\Sigma_{1}(t)}-H_{\Sigma_{2}(t)}\right) u=\int_{0}^{1}\left[\partial_{\vartheta}, H_{\Sigma(\vartheta ; t)}\right] u d \vartheta
$$

and from (3.2),

$$
\begin{aligned}
{\left[\partial_{\vartheta}, H_{\Sigma(\vartheta ; t)}\right] u } & =p . v \cdot \iint K\left(\xi^{\prime}-\xi\right)\left(\left(\partial_{\vartheta} \xi-\partial_{\vartheta} \xi^{\prime}\right) \times\left(\xi_{\beta^{\prime}}^{\prime} u_{\alpha^{\prime}}^{\prime}-\xi_{\alpha^{\prime}}^{\prime} u_{\beta^{\prime}}^{\prime}\right)\right) d \alpha^{\prime} d \beta^{\prime} \\
& =\sum_{i}\left[a_{i}, H_{\Sigma(\vartheta ; t)}\right] \mathfrak{D}_{i} u
\end{aligned}
$$

where $a_{i}$ are components of $\partial_{\vartheta} \xi=\xi_{1}-\xi_{2}$, that is, $\xi_{1}-\xi_{2}=\sum a_{i} e_{i}$, and $\mathfrak{D}_{i} u=$ $\frac{-\xi_{\alpha} \times \xi_{\beta}}{\left|\xi_{\alpha} \times \xi_{\beta}\right|^{2}} e_{i} \times\left(\xi_{\beta} u_{\alpha}-\xi_{\alpha} u_{\beta}\right)$, we have from Lemma $6.4 b$. that for $2+q \leq r \leq s+1 / 2$,

$$
\begin{aligned}
\left\|\left(H_{\Sigma_{1}(t)}-H_{\Sigma_{2}(t)}\right) u\right\|_{r} & \leq \int_{0}^{1}\left\|\left[\partial_{\vartheta}, H_{\Sigma(\vartheta ; t)}\right] u\right\|_{r} d \vartheta \leq \sum_{i} \int_{0}^{1}\left\|\left[a_{i}, H_{\Sigma(\vartheta ; t)}\right] \mathfrak{D}_{i} u\right\|_{r} d \vartheta \\
& \leq k_{r, 1}\left\|\xi_{1}(t)-\xi_{2}(t)\right\|_{r}\|u\|_{r}
\end{aligned}
$$

where $k_{r, 1}=k_{r, 1}\left(C_{0}, \mu, M(r)\right)$, and $\left\|\xi_{i, \alpha}-e_{\alpha}\right\|_{r-1}+\left\|\xi_{i, \beta}-e_{\beta}\right\|_{r-1} \leq M(r), i=1,2$. This proves Lemma 6.10 a.

For fixed $t$, let $\mathbf{n}_{i}=\frac{\xi_{i, \alpha} \times \xi_{i, \beta}}{\left|\xi_{i, \alpha} \times \xi_{i, \beta}\right|}$ be the unit normal of $\Sigma_{i}(t), i=1,2$. Using a similar argument and Lemma 3.2 we also have the following

Lemma 6.11. Let $t \in[0, T]$ be fixed. For $u \in \mathfrak{S}\left(R^{2}\right)$, we have

$$
\left\|\left(\nabla_{\mathbf{n}_{1}}-\nabla_{\mathbf{n}_{2}}\right) u\right\|_{r-1} \leq k_{r, 2}\left\|\xi_{1}(t)-\xi_{2}(t)\right\|_{r}\|u\|_{r}, \quad \text { for } 2+q \leq r \leq s+1 / 2
$$

where $k_{r, 2}=k_{r, 2}\left(C_{0}, \mu, M(r), M_{1}\right)$, and $\left\|\xi_{i, \alpha}-e_{\alpha}\right\|_{r-1}+\left\|\xi_{i, \beta}-e_{\beta}\right\|_{r-1} \leq M(r)$, for $i=1,2$, and $\sup _{0 \leq \vartheta \leq 1}\left\|\left(I+\mathcal{K}^{*}\right)^{-1}\right\| \leq M_{1}$, where $\mathcal{K}$ is the double layered potential defined on $\Sigma(\vartheta ; t)$.

An easy consequence of Lemmas 6.10 and 6.11 is the following

Lemma 6.12. Assume that $u \in C\left([0, T], \mathfrak{S}\left(R^{2}\right)\right)$, and $\Sigma(t)$ satisfies $H 1$, H2 and H3. Then $H_{\Sigma(t)} u \in C\left([0, T], H^{r}\left(R^{2}\right)\right)$, and $\nabla_{\mathbf{n}} u \in C\left([0, T], H^{r-1}\left(R^{2}\right)\right)$, for $2+q \leq$ $r \leq s+1 / 2$.

Linear systems. Assume that surfaces $\Sigma(t), t \in[0, T]$, are given and satisfy the conditions described at the beginning of the last part, in particular H1, H2 and $\mathrm{H} 3$; and assume $\nabla_{\mathbf{n}}$ is the normal derivative defined on $\Sigma(t)$. Assume that $\mathfrak{a}$ is a scalar-valued function such that $\mathfrak{a}-1 \in C\left([0, T], H^{s}\left(R^{2}\right)\right), \mathfrak{a}_{t} \in C\left([0, T], H^{1+q}\left(R^{2}\right)\right)$ and $\mathfrak{a} \geq c_{0}$ for some constant $c_{0}>0$. Let $a=\frac{\mathfrak{a}}{\left|\xi_{\alpha} \times \xi_{\beta}\right|}$, and let $G$ be the operator 
in (6.17), with $\lambda=\sup _{0 \leq t \leq T}\left(\left|\nabla_{\mathbf{n}} a\right|_{\infty}\left|\xi_{\alpha} \times \xi_{\beta}\right|_{\infty}+\frac{c_{0}}{\left|\xi_{\alpha} \times \xi_{\beta}\right|_{\infty}}\right)$. We know from Lemma 6.1, Corollary 6.5 and our assumption that $\lambda$ is a finite number. Let $G_{t}=\mathfrak{a}_{t} \nabla_{\mathbf{n}}+\mathfrak{a}\left[\partial_{t}, \nabla_{\mathbf{n}}\right], \Lambda=1+|D|$, and $(u, w)_{r}=\left(\Lambda^{r} u, \Lambda^{r} w\right)$. If $u$, $w$ are vector-valued or Clifford 1-vector-valued, we define $(u, w)=\iint u \cdot w d \alpha d \beta$, and $(u, w)_{r}=\left(\Lambda^{r} u, \Lambda^{r} w\right)$. For $\left(u, u_{t}\right) \in \mathfrak{S}\left(R^{2}\right) \times \mathfrak{S}\left(R^{2}\right), u, u_{t}$ scalar-valued or vectorvalued, we define

$$
\begin{aligned}
& E_{s, \epsilon}\left(u, u_{t}\right) \\
& \quad=\left(u_{t}, u_{t}\right)_{s-1}+\left(\nabla_{\mathbf{n}} u_{t}, \nabla_{\mathbf{n}} u_{t}\right)_{s-1}+\left(G \Lambda^{s-1} u, \Lambda^{s-1} u\right)+\left(G \Lambda^{s-1} \nabla_{\mathbf{n}} u, \Lambda^{s-1} \nabla_{\mathbf{n}} u\right) \\
& \quad+\epsilon^{2}\left\{\left(u_{\alpha}, u_{\alpha}\right)_{s-1}+\left(u_{\beta}, u_{\beta}\right)_{s-1}+\left(\nabla_{\mathbf{n}} u_{\alpha}, \nabla_{\mathbf{n}} u_{\alpha}\right)_{s-1}+\left(\nabla_{\mathbf{n}} u_{\beta}, \nabla_{\mathbf{n}} u_{\beta}\right)_{s-1}\right\}, \\
& E_{s}\left(u, u_{t}\right) \\
& \quad=\left(u_{t}, u_{t}\right)_{s-1}+\left(\nabla_{\mathbf{n}} u_{t}, \nabla_{\mathbf{n}} u_{t}\right)_{s-1}+\left(G \Lambda^{s-1} u, \Lambda^{s-1} u\right)+\left(G \Lambda^{s-1} \nabla_{\mathbf{n}} u, \Lambda^{s-1} \nabla_{\mathbf{n}} u\right), \\
& \left|\left(u, u_{t}\right)\right|_{s}^{2}=\|u\|_{s+1 / 2}^{2}+\left\|u_{t}\right\|_{s}^{2} .
\end{aligned}
$$

From Corollary 6.5 and Lemma 6.9, we know that

$$
\kappa^{-1}\left|\left(u, u_{t}\right)\right|_{s}^{2} \leq E_{s}\left(u, u_{t}\right) \leq \kappa\left|\left(u, u_{t}\right)\right|_{s}^{2}
$$

where $\kappa=\kappa\left(C_{0}, c_{0}, \mu, M\left(s+\frac{1}{2}\right), M_{1}, M_{2}\right)>1$ is a constant, and

$$
\begin{aligned}
& \sup _{0 \leq t \leq T}\left(\left\|\xi_{\alpha}-e_{\alpha}\right\|_{s-\frac{1}{2}}+\left\|\xi_{\beta}-e_{\beta}\right\|_{s-\frac{1}{2}}\right) \leq M\left(s+\frac{1}{2}\right), \\
& \sup _{0 \leq t \leq T}\left\|\left(I+\mathcal{K}^{*}\right)^{-1}\right\| \leq M_{1}, \quad \sup _{0 \leq t \leq T}\|\mathfrak{a}-1\|_{1+q} \leq M_{2} .
\end{aligned}
$$

In this part, we solve the initial value problem of the linear system

$$
\begin{aligned}
& u_{t t}+\mathfrak{a} \nabla_{\mathbf{n}} u=g, \quad 0 \leq t \leq T, \\
& u(\cdot, 0)=u_{0}, u_{t}(\cdot, 0)=u_{1} .
\end{aligned}
$$

We assume that $\mathfrak{a}$ and $g$ are scalar-valued, so we are only concerned with a scalar equation. The approach here is very much the same as for the $2-\mathrm{D}$ case [24]. We only give the main steps and present in detail those proofs which have major differences from the 2-D case.

As in the 2-D case, we first consider the linear systems:

$$
\begin{aligned}
& u_{t t}+\mathfrak{a} \nabla_{\mathbf{n}} u-\epsilon^{2} \Delta u=g, \quad 0 \leq t \leq T, \\
& u(\cdot, 0)=u_{0}, u_{t}(\cdot, 0)=u_{1}
\end{aligned}
$$

where $\Delta=\partial_{\alpha}^{2}+\partial_{\beta}^{2}$.

Let

$\mathrm{H}^{\prime \prime}: \xi_{\alpha}-e_{\alpha}, \xi_{\beta}-e_{\beta} \in C^{1}\left([0, T], H^{s}\left(R^{2}, \mathcal{C}\left(V_{3}\right)\right)\right), e_{\alpha} \times e_{\beta}=e_{3}$ and $\xi_{t} \in$ $C\left([0, T], H^{s+1 / 2}\left(R^{2}, \mathcal{C}\left(V_{3}\right)\right)\right)$.

Lemma 6.13. Let $0<\epsilon<1,0<T<\infty, s \geq 3 / 2+q, \mathfrak{a}-1 \in C\left([0, T], H^{s}\left(R^{2}\right)\right)$, and $g \in C\left([0, T], H^{s}\left(R^{2}\right)\right)$. Assume that $\Sigma(t), t \in[0, T]$, satisfies $H 1, H^{\prime \prime}$ and H3.

a. If $u_{0} \in H^{s+1}, u_{1} \in H^{s}$, then there exists a unique solution $u$ of $(6.26 \epsilon)$, such that $u \in C^{j}\left([0, T], H^{s+1-j}\right), j=0,1,2$.

b. Assume further that $\mathfrak{a}_{t} \in C\left([0, T], H^{1+q}\left(R^{2}\right)\right)$, and $\mathfrak{a} \geq c_{0}$, for some constant $c_{0}>0$. If $u \in C^{j}\left([0, T], H^{s+1-j}\right), j=0,1,2$, is a solution of $(6.26 \epsilon)$, then $u$ 
satisfies the estimate

$$
E_{s, \epsilon}\left(u, u_{t}\right) \leq e^{c t} E_{s, \epsilon}\left(u_{0}, u_{1}\right)+\int_{0}^{t} e^{c(t-\tau)}\|g\|_{s}^{2} d \tau
$$

for $0 \leq t \leq T$, where $c=c\left(C_{0}, c_{0}, \mu, M(s+1), M_{1}, M_{2}(s), M_{3}, M_{4}(s+1 / 2)\right)$ is a constant, and

$$
\begin{aligned}
\sup _{0 \leq t \leq T}\left(\left\|\xi_{\alpha}-e_{\alpha}\right\|_{s}+\left\|\xi_{\beta}-e_{\beta}\right\|_{s}\right) \leq M(s+1), & \sup _{0 \leq t \leq T}\|\mathfrak{a}-1\|_{s} \leq M_{2}(s), \\
\sup _{0 \leq t \leq T}\left\|\mathfrak{a}_{t}\right\|_{1+q} \leq M_{3}, & \sup _{0 \leq t \leq T}\left\|\xi_{t}\right\|_{s+1 / 2} \leq M_{4}(s+1 / 2),
\end{aligned}
$$

$M_{1}$ is as defined in (6.24).

Proof. a. can be proved in the same way as [24, Lemma 5.7 a]. We omit the proof. We prove (6.27) in detail for $u \in C^{j}\left([0, T], H^{s+2-j}\right), j=0,1,2$. For $u \in$ $C^{j}\left([0, T], H^{s+1-j}\right), j=0,1,2$, as stated in this lemma, we can obtain (6.27) as we did in [24] through an approximation argument.

Let $a=\frac{\mathfrak{a}}{\left|\xi_{\alpha} \times \xi_{\beta}\right|}$, so $\|a-1\|_{r} \leq c_{1}\|\mathfrak{a}-1\|_{r}$, for $1+q \leq r \leq s-1 / 2$, where $c_{1}=c_{1}\left(\mu,\left\|\xi_{\alpha}-e_{\alpha}\right\|_{r},\left\|\xi_{\beta}-e_{\beta}\right\|_{r}\right)$. Let $u \in C^{j}\left([0, T], H^{s+2-j}\right), j=0,1,2$, be a solution of $(6.26 \epsilon)$. We have

$$
\begin{aligned}
& \frac{d}{d t} E_{s, \epsilon}\left(u, u_{t}\right)=2\left(u_{t t}, u_{t}\right)_{s-1}+2\left(\nabla_{\mathbf{n}} u_{t t}, \nabla_{\mathbf{n}} u_{t}\right)_{s-1}+2\left(\left[\partial_{t}, \nabla_{\mathbf{n}}\right] u_{t}, \nabla_{\mathbf{n}} u_{t}\right)_{s-1} \\
& \quad+\left(G \Lambda^{s-1} u, \Lambda^{s-1} u_{t}\right)+\left(G \Lambda^{s-1} u_{t}, \Lambda^{s-1} u\right)+\left(G_{t} \Lambda^{s-1} u, \Lambda^{s-1} u\right) \\
& \quad+\left(G \Lambda^{s-1} \nabla_{\mathbf{n}} u, \Lambda^{s-1} \nabla_{\mathbf{n}} u_{t}\right)+\left(G \Lambda^{s-1} \nabla_{\mathbf{n}} u_{t}, \Lambda^{s-1} \nabla_{\mathbf{n}} u\right)+\left(G_{t} \Lambda^{s-1} \nabla_{\mathbf{n}} u, \Lambda^{s-1} \nabla_{\mathbf{n}} u\right) \\
& \quad+\left(G \Lambda^{s-1}\left[\partial_{t}, \nabla_{\mathbf{n}}\right] u, \Lambda^{s-1} \nabla_{\mathbf{n}} u\right)+\left(G \Lambda^{s-1} \nabla_{\mathbf{n}} u, \Lambda^{s-1}\left[\partial_{t}, \nabla_{\mathbf{n}}\right] u\right) \\
& \quad+2 \epsilon^{2}\left(u_{t \alpha}, u_{\alpha}\right)_{s-1}+2 \epsilon^{2}\left(u_{t \beta}, u_{\beta}\right)_{s-1}+2 \epsilon^{2}\left(\nabla_{\mathbf{n}} u_{t \alpha}, \nabla_{\mathbf{n}} u_{\alpha}\right)_{s-1} \\
& \quad+2 \epsilon^{2}\left(\nabla_{\mathbf{n}} u_{t \beta}, \nabla_{\mathbf{n}} u_{\beta}\right)_{s-1}+2 \epsilon^{2}\left(\left[\partial_{t}, \nabla_{\mathbf{n}}\right] u_{\alpha}, \nabla_{\mathbf{n}} u_{\alpha}\right)_{s-1}+2 \epsilon^{2}\left(\left[\partial_{t}, \nabla_{\mathbf{n}}\right] u_{\beta}, \nabla_{\mathbf{n}} u_{\beta}\right)_{s-1} \\
& \quad=I_{1}+I_{2}+I_{3}+I_{4}+I_{5}
\end{aligned}
$$

where

$$
\begin{aligned}
& I_{1}= 2\left(u_{t t}, u_{t}\right)_{s-1}+\left(G \Lambda^{s-1} u, \Lambda^{s-1} u_{t}\right)+\left(G \Lambda^{s-1} u_{t}, \Lambda^{s-1} u\right) \\
& \quad+2 \epsilon^{2}\left(u_{t \alpha}, u_{\alpha}\right)_{s-1}+2 \epsilon^{2}\left(u_{t \beta}, u_{\beta}\right)_{s-1}, \\
& I_{2}=2\left(\nabla_{\mathbf{n}} u_{t t}, \nabla_{\mathbf{n}} u_{t}\right)_{s-1}+\left(G \Lambda^{s-1} \nabla_{\mathbf{n}} u, \Lambda^{s-1} \nabla_{\mathbf{n}} u_{t}\right)+\left(G \Lambda^{s-1} \nabla_{\mathbf{n}} u_{t}, \Lambda^{s-1} \nabla_{\mathbf{n}} u\right) \\
& \quad+2 \epsilon^{2}\left(\nabla_{\mathbf{n}} u_{t \alpha}, \nabla_{\mathbf{n}} u_{\alpha}\right)_{s-1}+2 \epsilon^{2}\left(\nabla_{\mathbf{n}} u_{t \beta}, \nabla_{\mathbf{n}} u_{\beta}\right)_{s-1}, \\
& I_{3}=2\left(\left[\partial_{t}, \nabla_{\mathbf{n}}\right] u_{t}, \nabla_{\mathbf{n}} u_{t}\right)_{s-1}+2 \epsilon^{2}\left(\left[\partial_{t}, \nabla_{\mathbf{n}}\right] u_{\alpha}, \nabla_{\mathbf{n}} u_{\alpha}\right)_{s-1}+2 \epsilon^{2}\left(\left[\partial_{t}, \nabla_{\mathbf{n}}\right] u_{\beta}, \nabla_{\mathbf{n}} u_{\beta}\right)_{s-1}, \\
& I_{4}=\left(G \Lambda^{s-1}\left[\partial_{t}, \nabla_{\mathbf{n}}\right] u, \Lambda^{s-1} \nabla_{\mathbf{n}} u\right)+\left(G \Lambda^{s-1} \nabla_{\mathbf{n}} u, \Lambda^{s-1}\left[\partial_{t}, \nabla_{\mathbf{n}}\right] u\right), \\
& I_{5}=\left(G_{t} \Lambda^{s-1} u, \Lambda^{s-1} u\right)+\left(G_{t} \Lambda^{s-1} \nabla_{\mathbf{n}} u, \Lambda^{s-1} \nabla_{\mathbf{n}} u\right) .
\end{aligned}
$$

From Lemmas 3.1, 3.2, 6.2 and 6.4, we know

$$
\left\|\left[\partial_{t}, \nabla_{\mathbf{n}}\right] w\right\|_{r-1} \leq k_{r}^{\prime}\left(\left\|\xi_{t}\right\|_{r-1}\|w\|_{r}+\left\|\xi_{t}\right\|_{r}\|w\|_{1+q}\right) \quad \text { for } r=s, s+1 / 2,
$$

where $k_{r}^{\prime}=k_{r}^{\prime}\left(C_{0}, \mu, M(r), M_{1}\right)$, and $\sup _{0 \leq t \leq T}\left(\left\|\xi_{\alpha}-e_{\alpha}\right\|_{r-1}+\left\|\xi_{\beta}-e_{\beta}\right\|_{r-1}\right) \leq$ $M(r), r=s, s+1 / 2$. Therefore using Corollary 6.5, we get

$$
\left|I_{3}\right| \leq k_{s}^{\prime}\left\|\xi_{t}\right\|_{s}\left(\left\|u_{t}\right\|_{1+q}\left\|u_{t}\right\|_{s}+\epsilon^{2}\left\|u_{\alpha}\right\|_{s}^{2}+\epsilon^{2}\left\|u_{\beta}\right\|_{s}^{2}\right)+k_{s}^{\prime}\left\|\xi_{t}\right\|_{s-1}\left\|u_{t}\right\|_{s}^{2}
$$


and using Lemma 6.6 a, Corollary 6.5, Lemma 6.7 and (6.29), we have

$$
\begin{aligned}
\left|I_{4}\right| \leq \mid \int_{\Sigma(t)}\left\{a\left(\Lambda^{s-1} \nabla_{\mathbf{n}} u\right) \nabla_{\mathbf{n}}\left(\Lambda^{s-1}\left[\partial_{t}, \nabla_{\mathbf{n}}\right] u\right)\right. \\
\left.\quad+a\left(\Lambda^{s-1}\left[\partial_{t}, \nabla_{\mathbf{n}}\right] u\right) \nabla_{\mathbf{n}}\left(\Lambda^{s-1} \nabla_{\mathbf{n}} u\right)\right\} d S \mid \\
+2 \lambda\left|\left(\Lambda^{s-1}\left[\partial_{t}, \nabla_{\mathbf{n}}\right] u, \Lambda^{s-1} \nabla_{\mathbf{n}} u\right)\right| \\
\leq k_{s+1 / 2}^{\prime}\left(\|\mathfrak{a}-1\|_{1+q}+1\right)\left(\left\|\xi_{t}\right\|_{s-1 / 2}\|u\|_{s+1 / 2}^{2}+\left\|\xi_{t}\right\|_{s+1 / 2}\|u\|_{1+q}\|u\|_{s+1 / 2}\right) .
\end{aligned}
$$

Since

$$
\begin{aligned}
I_{5}= & \int_{\Sigma(t)} \frac{\mathfrak{a}_{t}}{\left|\xi_{\alpha} \times \xi_{\beta}\right|}\left(\Lambda^{s-1} u\right) \nabla_{\mathbf{n}}\left(\Lambda^{s-1} u\right) d S \\
& +\int_{\Sigma(t)} \frac{\mathfrak{a}_{t}}{\left|\xi_{\alpha} \times \xi_{\beta}\right|}\left(\Lambda^{s-1} \nabla_{\mathbf{n}} u\right) \nabla_{\mathbf{n}}\left(\Lambda^{s-1} \nabla_{\mathbf{n}} u\right) d S \\
& +\int_{\Sigma(t)} a\left(\left[\partial_{t}, \nabla_{\mathbf{n}}\right] \Lambda^{s-1} u\right)\left(\Lambda^{s-1} u\right) d S+\int_{\Sigma(t)} a\left(\left[\partial_{t}, \nabla_{\mathbf{n}}\right] \Lambda^{s-1} \nabla_{\mathbf{n}} u\right)\left(\Lambda^{s-1} \nabla_{\mathbf{n}} u\right) d S,
\end{aligned}
$$

we have from Lemma 6.6 a, Corollary 6.5 , Lemma 6.7 and 6.8 that

$$
\left|I_{5}\right| \leq k_{s+1 / 2}^{\prime}\left(\left\|\mathfrak{a}_{t}\right\|_{1+q}+1\right)\|u\|_{s+1 / 2}^{2}+k_{s+1 / 2}^{\prime}\left(\|\mathfrak{a}-1\|_{1+q}+1\right)\left\|\xi_{t}\right\|_{2+q}\|u\|_{s+1 / 2}^{2} .
$$

We remark that the constants $k_{r}^{\prime}, r=s, s+1 / 2, s+1$, in (6.29)-(6.32) and in the following are not the same, but depend on the same factors.

We now estimate $I_{2}$. Since $u$ is a solution of $(6.26 \epsilon)$, we have

$$
\begin{aligned}
I_{2}= & 2\left(\nabla_{\mathbf{n}}\left(-\mathfrak{a} \nabla_{\mathbf{n}} u\right), \nabla_{\mathbf{n}} u_{t}\right)_{s-1}+2\left(\nabla_{\mathbf{n}} g, \nabla_{\mathbf{n}} u_{t}\right)_{s-1}+\left(\mathfrak{a} \nabla_{\mathbf{n}} \Lambda^{s-1} \nabla_{\mathbf{n}} u, \Lambda^{s-1} \nabla_{\mathbf{n}} u_{t}\right) \\
& +\left(\mathfrak{a} \nabla_{\mathbf{n}} \Lambda^{s-1} \nabla_{\mathbf{n}} u_{t}, \Lambda^{s-1} \nabla_{\mathbf{n}} u\right)+2 \lambda\left(\Lambda^{s-1} \nabla_{\mathbf{n}} u_{t}, \Lambda^{s-1} \nabla_{\mathbf{n}} u\right) \\
& +2 \epsilon^{2}\left(\left[\nabla_{\mathbf{n}}, \partial_{\alpha}\right] u_{t}, \nabla_{\mathbf{n}} u_{\alpha}\right)_{s-1}+2 \epsilon^{2}\left(\left[\nabla_{\mathbf{n}}, \partial_{\beta}\right] u_{t}, \nabla_{\mathbf{n}} u_{\beta}\right)_{s-1} \\
& -2 \epsilon^{2}\left(\nabla_{\mathbf{n}} u_{t},\left[\partial_{\alpha}, \nabla_{\mathbf{n}}\right] u_{\alpha}\right)_{s-1}-2 \epsilon^{2}\left(\nabla_{\mathbf{n}} u_{t},\left[\partial_{\beta}, \nabla_{\mathbf{n}}\right] u_{\beta}\right)_{s-1} \\
& =2\left(-\Lambda^{s-1} \nabla_{\mathbf{n}}\left(\mathfrak{a} \nabla_{\mathbf{n}} u\right)+\mathfrak{a} \nabla_{\mathbf{n}} \Lambda^{s-1} \nabla_{\mathbf{n}} u, \Lambda^{s-1} \nabla_{\mathbf{n}} u_{t}\right)+I_{2}^{\prime},
\end{aligned}
$$

where

$$
\begin{aligned}
I_{2}^{\prime}= & \int_{\Sigma(t)}\left[a, \nabla_{\mathbf{n}}\right]\left(\Lambda^{s-1} \nabla_{\mathbf{n}} u_{t}\right)\left(\Lambda^{s-1} \nabla_{\mathbf{n}} u\right) d S+2 \lambda\left(\Lambda^{s-1} \nabla_{\mathbf{n}} u_{t}, \Lambda^{s-1} \nabla_{\mathbf{n}} u\right) \\
& +2\left(\nabla_{\mathbf{n}} g, \nabla_{\mathbf{n}} u_{t}\right)_{s-1}+2 \epsilon^{2}\left(\left[\nabla_{\mathbf{n}}, \partial_{\alpha}\right] u_{t}, \nabla_{\mathbf{n}} u_{\alpha}\right)_{s-1}+2 \epsilon^{2}\left(\left[\nabla_{\mathbf{n}}, \partial_{\beta}\right] u_{t}, \nabla_{\mathbf{n}} u_{\beta}\right)_{s-1} \\
& -2 \epsilon^{2}\left(\nabla_{\mathbf{n}} u_{t},\left[\partial_{\alpha}, \nabla_{\mathbf{n}}\right] u_{\alpha}\right)_{s-1}-2 \epsilon^{2}\left(\nabla_{\mathbf{n}} u_{t},\left[\partial_{\beta}, \nabla_{\mathbf{n}}\right] u_{\beta}\right)_{s-1} .
\end{aligned}
$$

We know from Lemma 6.6 c, Corollary 6.5, and Lemmas 6.7, 6.4 and 3.2, that

$$
\begin{aligned}
\left|I_{2}^{\prime}\right| & \leq k_{s+1 / 2}^{\prime}\left(\|\mathfrak{a}-1\|_{1+q}+1\right)\left\|u_{t}\right\|_{s}\|u\|_{s+1 / 2}+k_{s+1 / 2}^{\prime}\|g\|_{s}\left\|u_{t}\right\|_{s} \\
& +\epsilon^{2} k_{s+1}^{\prime}\left\|u_{t}\right\|_{s}\left(\left\|u_{\alpha}\right\|_{s}+\left\|u_{\beta}\right\|_{s}\right) \\
& \leq k_{s+1 / 2}^{\prime}\left(\|\mathfrak{a}-1\|_{1+q}+1\right)\left(\left\|u_{t}\right\|_{s}^{2}+\|u\|_{s+1 / 2}^{2}\right)+k_{s+1 / 2}^{\prime}\left\|u_{t}\right\|_{s}^{2} \\
& +\|g\|_{s}^{2}+\epsilon^{4} k_{s+1}^{\prime}\left(\left\|u_{\alpha}\right\|_{s}^{2}+\left\|u_{\beta}\right\|_{s}^{2}\right),
\end{aligned}
$$


where $k_{s+1}^{\prime}=k_{s+1}^{\prime}\left(C_{0}, \mu, M(s+1), M_{1}\right)$. On the other hand,

$$
\begin{aligned}
-\Lambda^{s-1} \nabla_{\mathbf{n}}\left(\mathfrak{a} \nabla_{\mathbf{n}} u\right)+\mathfrak{a} \nabla_{\mathbf{n}} \Lambda^{s-1} \nabla_{\mathbf{n}} u & =-\left[\Lambda^{s-1}, \nabla_{\mathbf{n}}\right]\left(\mathfrak{a} \nabla_{\mathbf{n}} u\right) \\
& +\nabla_{\mathbf{n}}\left[\mathfrak{a}, \Lambda^{s-1}\right] \nabla_{\mathbf{n}} u+\left[\mathfrak{a}, \nabla_{\mathbf{n}}\right] \Lambda^{s-1} \nabla_{\mathbf{n}} u
\end{aligned}
$$

From a similar argument as in Lemma 6.4, we have

$$
\left\|\left[\Lambda^{s-1}, \nabla_{\mathbf{n}}\right]\left(\mathfrak{a} \nabla_{\mathbf{n}} u\right)\right\| \leq k_{s+1 / 2}^{\prime}\left(\left(\|\mathfrak{a}-1\|_{1+q}+1\right)\|u\|_{s}+\|\mathfrak{a}-1\|_{s-1}\|u\|_{1+q}\right),
$$

while from Lemma 6.2 and Corollary 6.5,

$$
\left\|\nabla_{\mathbf{n}}\left[\mathfrak{a}, \Lambda^{s-1}\right] \nabla_{\mathbf{n}} u\right\| \leq k_{s+1 / 2}^{\prime}\left(\|\mathfrak{a}-1\|_{1+q}\|u\|_{s}+\|\mathfrak{a}-1\|_{s}\|u\|_{1+q}\right) .
$$

Therefore

$$
\begin{aligned}
& \left|\left(-\Lambda^{s-1} \nabla_{\mathbf{n}}\left(\mathfrak{a} \nabla_{\mathbf{n}} u\right)+\mathfrak{a} \nabla_{\mathbf{n}} \Lambda^{s-1} \nabla_{\mathbf{n}} u, \Lambda^{s-1} \nabla_{\mathbf{n}} u_{t}\right)\right| \\
& \quad \leq k_{s+1 / 2}^{\prime}\left(\left(\|\mathfrak{a}-1\|_{1+q}+1\right)\|u\|_{s}+\|\mathfrak{a}-1\|_{s}\|u\|_{1+q}\right)\left\|u_{t}\right\|_{s} \\
& \quad+\left|\left(\left[\mathfrak{a}, \nabla_{\mathbf{n}}\right] \Lambda^{s-1} \nabla_{\mathbf{n}} u, \Lambda^{s-1} \nabla_{\mathbf{n}} u_{t}\right)\right| \\
& \quad \leq k_{s+1 / 2}^{\prime}\left(\left(\|\mathfrak{a}-1\|_{1+q}+1\right)\|u\|_{s+1 / 2}+\|\mathfrak{a}-1\|_{s}\|u\|_{1+q}\right)\left\|u_{t}\right\|_{s}
\end{aligned}
$$

where in the last inequality we used Lemma 6.6 c. Combining (6.33) and (6.34), we get

$$
\begin{aligned}
\left|I_{2}\right| \leq & k_{s+1 / 2}^{\prime}\left(\|\mathfrak{a}-1\|_{1+q}+1\right)\left(\left\|u_{t}\right\|_{s}^{2}+\|u\|_{s+1 / 2}^{2}\right)+k_{s+1 / 2}^{\prime}\|\mathfrak{a}-1\|_{s}\|u\|_{1+q}\left\|u_{t}\right\|_{s} \\
& +\|g\|_{s}^{2}+\epsilon^{4} k_{s+1}^{\prime}\left(\left\|u_{\alpha}\right\|_{s}^{2}+\left\|u_{\beta}\right\|_{s}^{2}\right) .
\end{aligned}
$$

Finally using a similar but much easier argument we have that

$$
\begin{aligned}
\left|I_{1}\right| \leq & k_{s+1 / 2}^{\prime}\left(\|\mathfrak{a}-1\|_{1+q}+1\right)\left(\left\|u_{t}\right\|_{s-1}^{2}+\|u\|_{s}^{2}\right) \\
& +k_{s+1 / 2}^{\prime}\|\mathfrak{a}-1\|_{s-1}\|u\|_{1+q}\left\|u_{t}\right\|_{s-1}+\|g\|_{s-1}^{2} .
\end{aligned}
$$

Combining (6.28) and (6.30)-(6.36), we obtain

$$
\begin{aligned}
\frac{d}{d t} E_{s, \epsilon}\left(u, u_{t}\right) & \leq c^{\prime}\left(\left\|u_{t}\right\|_{s}^{2}+\|u\|_{s+1 / 2}^{2}+\epsilon^{2}\left\|u_{\alpha}\right\|_{s}^{2}+\epsilon^{2}\left\|u_{\beta}\right\|_{s}^{2}\right)+\|g\|_{s}^{2} \\
& \leq c E_{s, \epsilon}\left(u, u_{t}\right)+\|g\|_{s}^{2}
\end{aligned}
$$

where

$$
c=c\left(C_{0}, c_{0}, \mu, M(s+1), M_{1}, \sup _{0 \leq t \leq T}\|\mathfrak{a}-1\|_{s}, \sup _{0 \leq t \leq T}\left\|\mathfrak{a}_{t}\right\|_{1+q}, \sup _{0 \leq t \leq T}\left\|\xi_{t}\right\|_{s+1 / 2}\right),
$$

and $c^{\prime}$ depends on the same factors as $c$. Therefore

$$
E_{s, \epsilon}\left(u, u_{t}\right) \leq e^{c t} E_{s, \epsilon}\left(u_{0}, u_{1}\right)+\int_{0}^{t} e^{c(t-\tau)}\|g\|_{s}^{2} d \tau
$$

This proves $(6.27)$ for $u \in C^{j}\left([0, T], H^{s+2-j}\right), j=0,1,2$. For $u \in C^{j}\left([0, T], H^{s+1-j}\right)$, $j=0,1,2,(6.27)$ is obtained through an approximation argument.

Taking $\epsilon=0$ in the above proof, we get the following estimates for solutions of $(6.25)$.

Lemma 6.14. Let $0<T<\infty, s \geq 3 / 2+q$, a $-1 \in C\left([0, T], H^{s}\left(R^{2}\right)\right)$, and $g \in C\left([0, T], H^{s}\left(R^{2}\right)\right)$. Assume that $\Sigma(t), t \in[0, T]$, satisfies H1, H2 and H3, and 
also that $\mathfrak{a}_{t} \in C\left([0, T], H^{1+q}\left(R^{2}\right)\right), \mathfrak{a} \geq c_{0}$, for some constant $c_{0}>0$. If $u \in$ $C^{j}\left([0, T], H^{s+1 / 2-j / 2}\left(R^{2}\right)\right), j=0,1,2$, satisfies (6.25), then

$$
\left|\left(u(t), u_{t}(t)\right)\right|_{s}^{2} \leq \kappa^{2} e^{d_{1} t}\left|\left(u_{0}, u_{1}\right)\right|_{s}^{2}+\kappa \int_{0}^{t} e^{d_{1}(t-\tau)}\|g(\tau)\|_{s}^{2} d \tau
$$

if further $s \geq 2+q$, then

$$
\begin{aligned}
\left|\left(u(t), u_{t}(t)\right)\right|_{s}^{2} \leq & \kappa^{2} e^{d_{2} t}\left|\left(u_{0}, u_{1}\right)\right|_{s}^{2} \\
& +\kappa \int_{0}^{t} e^{d_{2}(t-\tau)}\left(\|\mathfrak{a}-1\|_{s}^{2}+\left\|\xi_{t}\right\|_{s+1 / 2}^{2}+\|g(\tau)\|_{s}^{2}\right) d \tau
\end{aligned}
$$

where $\kappa$ is as in (6.24),

$$
\begin{aligned}
& d_{1}=d_{1}\left(C_{0}, c_{0}, \mu, M(s+1 / 2), M_{1}, M_{2}(s), M_{3}, M_{4}(s+1 / 2)\right), \\
& d_{2}=d_{2}\left(C_{0}, c_{0}, \mu, M(s+1 / 2), M_{1}, M_{2}, M_{3}, M_{4}(s), \sup _{0 \leq t \leq T}\|u\|_{1+q}\right)
\end{aligned}
$$

are constants, $M_{1}, M_{2}, M_{3}, M(s+1 / 2), M_{2}(s), M_{4}(s+1 / 2)$ are as in (6.24) and Lemma 6.13, and $\sup _{0 \leq t \leq T}\left\|\xi_{t}\right\|_{s} \leq M_{4}(s)$.

As in the 2-D case [24], we need the following result to guarantee that when passing to limits, we will get solutions with the desired regularity.

Lemma 6.15. Let $0<T<\infty, s \geq 3 / 2+q$, a $-1 \in L^{\infty}\left([0, T], H^{s}\right), g \in$ $L^{\infty}\left([0, T], H^{s}\right)$, and $\mathfrak{a}-1, \mathfrak{a}_{t} \in C\left([0, T], H^{1+q}\right)$. Assume that $\Sigma(t), t \in[0, T]$, satisfies H1, H2' and H3, and $\mathfrak{a} \geq c_{0}$ for some constant $c_{0}>0$. If $\left(u, u_{t}\right) \in$ $L^{\infty}\left([0, T], H^{s+1 / 2} \times H^{s}\right) \cap C\left([0, T], H^{s^{\prime}+1 / 2} \times H^{s^{\prime}}\right), s^{\prime}<s$, satisfies (6.25), then $\left(u, u_{t}\right) \in C\left([0, T], H^{s+1 / 2} \times H^{s}\right)$.

Proof. The proof of Lemma 6.15 is very similar to that of [24, Lemma 5.9], except that in this proof, we need to apply Lemma 6.11 to $\Sigma_{1}(t)=\Sigma(t)$ and $\Sigma_{2}(t)=$ $\Sigma\left(t+t^{\prime}\right)$, as $t^{\prime} \rightarrow 0$. We omit the details.

Finally we have the following well-posedness result for the linear system (6.25).

Theorem 6.16. Let $0<T<\infty, s \geq 5 / 2+q, \mathfrak{a}-1 \in C\left([0, T], H^{s}\left(R^{2}\right)\right)$, $\mathfrak{a}_{t} \in C\left([0, T], H^{1+q}\left(R^{2}\right)\right)$ and $g \in C\left([0, T], H^{s}\left(R^{2}\right)\right)$. Assume that $\Sigma(t), t \in[0, T]$, satisfies H1, H2 and H3, and $\mathfrak{a} \geq c_{0}$, for some constant $c_{0}>0$. If $\left(u_{0}, u_{1}\right) \in$ $H^{s+1 / 2} \times H^{s}$, then there is a unique solution $u$ of the linear system (6.25), such that $u \in C^{j}\left([0, T], H^{s+1 / 2-j / 2}\left(R^{2}\right)\right), j=0,1,2$.

Proof. The uniqueness of solutions is a consequence of Lemma 6.14. The proof of existence has two steps and is again very similar to that of [24, Theorem 5.10]. In Step 1, we assume further that $\Sigma(t)$ satisfies $\mathrm{H}_{2}^{\prime \prime}$ and $u_{0} \in H^{s+1}$, and we obtain the solution of (6.25) by taking the limit of solutions of $(6.26 \epsilon)$ as $\epsilon \rightarrow 0$. We use estimates (6.27) and (6.37) to show that the limit exists. We use Lemma 6.15 to ensure that the solution has the desired regularity.

In Step 2, we remove the further assumption made in Step 1 by smoothing $u_{0}$ and $\Sigma(t)$, then pass to the limit. The smoothing can be done as usual, that is, taking $\Sigma_{\delta}(t): \xi_{\delta}(t)=\xi(t) * \phi_{\delta}$ and $u_{0, \delta}=u_{0} * \phi_{\delta}$, where $\phi_{\delta}(\alpha, \beta)=\frac{1}{\delta^{2}} \phi\left(\frac{\alpha}{\delta}, \frac{\beta}{\delta}\right)$, $\phi \in \mathfrak{S}\left(R^{2}\right), \int \phi=1$. It is not hard to see that $\Sigma_{\delta}(t)$ satisfies $\mathrm{H} 2^{\prime \prime}$ and $u_{0, \delta} \in H^{s+1}$. We verify in the following that $\Sigma_{\delta}(t)$ also satisfies $\mathrm{H} 1$ and $\mathrm{H} 3$, when $\delta \leq \delta_{0}$, where $\delta_{0}>0$ is a constant. 
Let $\left(\alpha^{\prime}, \beta^{\prime}\right)=(\alpha, \beta)+\left(\alpha_{1}, \beta_{1}\right)$. Since

$$
\begin{aligned}
& \left(\xi_{\delta}\left(\alpha^{\prime}, \beta^{\prime}\right)-\xi_{\delta}(\alpha, \beta)\right)-\left(\xi\left(\alpha^{\prime}, \beta^{\prime}\right)-\xi(\alpha, \beta)\right) \\
& \quad=\int_{0}^{1} \frac{d}{d \theta}\left\{\left(\xi_{\delta}-\xi\right)\left(\alpha+\theta \alpha_{1}, \beta+\theta \beta_{1}\right)\right\} d \theta \\
& \quad=\int_{0}^{1}\left(\nabla \xi_{\delta}-\nabla \xi\right)\left(\alpha+\theta \alpha_{1}, \beta+\theta \beta_{1}\right) \cdot\left(\alpha_{1}, \beta_{1}\right) d \theta
\end{aligned}
$$

and since

$$
\left|\nabla \xi_{\delta}-\nabla \xi\right|_{\infty} \leq \delta C\left(\left\|\xi_{\alpha}-e_{\alpha}\right\|_{1+q}+\left\|\xi_{\beta}-e_{\beta}\right\|_{1+q}\right) \leq \delta C M
$$

we have

$$
\begin{aligned}
\left|\xi_{\delta}\left(\alpha^{\prime}, \beta^{\prime}\right)-\xi_{\delta}(\alpha, \beta)\right| & \geq\left|\xi\left(\alpha^{\prime}, \beta^{\prime}\right)-\xi(\alpha, \beta)\right|-\delta C M\left(\left|\alpha_{1}\right|+\left|\beta_{1}\right|\right) \\
& \geq\left(C_{0}-\delta C M\right)\left(\left|\alpha-\alpha^{\prime}\right|+\left|\beta-\beta^{\prime}\right|\right)
\end{aligned}
$$

where $C$ is a universal constant, $\left\|\xi_{\alpha}-e_{\alpha}\right\|_{1+q}+\left\|\xi_{\beta}-e_{\beta}\right\|_{1+q} \leq M$. Consequently

$$
\left|\xi_{\delta}\left(\alpha^{\prime}, \beta^{\prime}\right)-\xi_{\delta}(\alpha, \beta)\right| \geq \frac{C_{0}}{2}\left(\left|\alpha-\alpha^{\prime}\right|+\left|\beta-\beta^{\prime}\right|\right)
$$

for $0<\delta \leq \frac{C_{0}}{2 C M}$. The fact that $\xi_{\delta}$, for $\delta \leq \delta_{0}$, satisfies $\mathrm{H} 3$ can be verified similarly. The rest of proof is carried out similar to that of [24, Theorem 5.10].

It is clear that Lemmas 6.14 and 6.15 and Theorem 6.16 also hold if $g, u_{0}, u_{1}$ and the solution $u$ are vector-valued or Clifford 1-vector-valued.

The quasilinear system. Let $\mathfrak{g}\left(\xi, \mathfrak{u}, \mathfrak{u}_{t}\right)=-\tilde{\mathbf{n}}\left(I+\widetilde{\mathcal{K}^{*}}\right)^{-1}\left(\Re\left\{\tilde{\mathbf{n}} f\left(\xi, \mathfrak{u}, \mathfrak{u}_{t}\right)\right\}\right)$, where $f=f\left(\xi, \mathfrak{u}, \mathfrak{u}_{t}\right), \tilde{\mathbf{n}}$ are as defined in $(5.22)$ and $\widetilde{\mathcal{K}^{*}}$ is as defined in (5.23). The following estimates of $\mathfrak{g}$ and $\mathfrak{a}$ are easy consequences of results in the estimates part.

Lemma 6.17. Let $0<T<\infty, s \geq 3 / 2+q$. Assume that for $t \in[0, T], \Sigma(t)$ : $\xi=\xi(\alpha, \beta, t)$ satisfies $H 1, H 2$ and $H 3$, and $\left(I+\widetilde{\mathcal{K}^{*}}\right)^{-1}$ is bounded from $L_{2}\left(R^{2}\right)$ to $L_{2}\left(R^{2}\right)$; assume further that $\mathfrak{u}, \mathfrak{u}_{t} \in C\left([0, T], H^{s}\left(R^{2}, \mathcal{C}\left(V_{3}\right)\right)\right)$, and $\left|\mathfrak{u}_{t}+e_{3}\right| \geq c_{0}$, for some constant $c_{0}>0$. Then

a. $\mathfrak{g}=\mathfrak{g}\left(\xi, \mathfrak{u}, \mathfrak{u}_{t}\right) \in C\left([0, T], H^{s}\left(R^{2}, C\left(V_{3}\right)\right)\right)$, and

$$
\|\mathfrak{g}\|_{s} \leq K_{g}\left(C_{0}, c_{0}, \mu, M(s), \widetilde{M}_{1}, \mathcal{M}(s), \mathcal{M}_{t}(s)\right),
$$

where $K_{g}=K_{g}\left(C_{0}, c_{0}, \mu, M(s), \widetilde{M}_{1}, \mathcal{M}(s), \mathcal{M}_{t}(s)\right)$ is a constant,

$$
\sup _{0 \leq t \leq T}\left(\left\|\xi_{\alpha}-e_{\alpha}\right\|_{s-1}+\left\|\xi_{\beta}-e_{\beta}\right\|_{s-1}\right) \leq M(s), \sup _{0 \leq t \leq T}\left\|\left(I+\widetilde{\mathcal{K}^{*}}\right)^{-1}\right\| \leq \widetilde{M}_{1},
$$

with $\left\|\left(I+\widetilde{\mathcal{K}^{*}}\right)^{-1}\right\|$ the operator norm of $\left(I+\widetilde{\mathcal{K}^{*}}\right)^{-1}$ from $L_{2}\left(R^{2}\right)$ to $L_{2}\left(R^{2}\right)$, and

$$
\sup _{0 \leq t \leq T}\|\mathfrak{u}\|_{s} \leq \mathcal{M}(s), \quad \sup _{0 \leq t \leq T}\left\|\mathfrak{u}_{t}\right\|_{s} \leq \mathcal{M}_{t}(s) .
$$

b. $\mathfrak{a}-1=\left|\mathfrak{u}_{t}+e_{3}\right|-1 \in C\left([0, T], H^{s}\left(R^{2}\right)\right)$, and

$$
\|\mathfrak{a}-1\|_{r} \leq K_{a}\left(\mathcal{M}_{t}(r)\right), \quad \text { for } 1+q \leq r \leq s,
$$

where $K_{a}=K_{a}\left(\mathcal{M}_{t}(r)\right)$ is a constant, $\sup _{0 \leq t \leq T}\left\|\mathfrak{u}_{t}\right\|_{r} \leq \mathcal{M}_{t}(r)$.

c. Assume further that $\mathfrak{u}_{t t} \in C\left([0, T], H^{1+q}\left(R^{2}, \mathcal{C}\left(V_{3}\right)\right)\right)$. Then

$$
\mathfrak{a}_{t} \in C\left([0, T], H^{1+q}\left(R^{2}\right)\right),
$$


and

$$
\left\|\mathfrak{a}_{t}\right\|_{1+q} \leq K_{a_{t}}\left(\mathcal{M}_{t}(1+q), \mathcal{M}_{t t}\right),
$$

where $K_{a_{t}}=K_{a_{t}}\left(\mathcal{M}_{t}(1+q), \mathcal{M}_{t t}\right)$ is a constant, $\sup _{0 \leq t \leq T}\left\|\mathfrak{u}_{t t}\right\|_{1+q} \leq \mathcal{M}_{t t}$, and $\mathcal{M}_{t}(1+q)$ is as defined in $b$.

Proof. $b$. and $c$. are easy consequences of Lemmas 6.1 and 6.2.

Notice that $\tilde{\mathbf{n}}=\frac{\mathfrak{u}_{t}+e_{3}}{\left|\mathfrak{u}_{t}+e_{3}\right|} \in C\left([0, T], H^{s}\left(R^{2}, \mathcal{C}\left(V_{3}\right)\right)\right)$. From the same proof for Lemma $6.4 d$, we have $\left(I+\widetilde{\mathcal{K}^{*}}\right)^{-1}$ is bounded from $H^{s}\left(R^{2}\right)$ to $H^{s}\left(R^{2}\right)$. Notice further that each term in $f\left(\xi, \mathfrak{u}, \mathfrak{u}_{t}\right)$ is an operator of one of the types discussed in Lemma 6.4, with $a, b=\mathfrak{u}$ or $\mathfrak{u}_{t}$, therefore we have $a$.

The following result is a consequence of Lemmas 6.4 and 6.10.

Lemma 6.18. Let $s \geq 3 / 2+q$. For $t \in[0, T]$ and $0 \leq \vartheta \leq 1$, let

$$
\begin{aligned}
& \Sigma_{1}(t): \xi^{1}=\xi^{1}(\alpha, \beta, t), \quad \Sigma_{2}(t): \xi^{2}=\xi^{2}(\alpha, \beta, t), \\
& \Sigma(\vartheta ; t): \xi=\vartheta \xi^{1}(\alpha, \beta, t)+(1-\vartheta) \xi^{2}(\alpha, \beta, t) .
\end{aligned}
$$

Assume that $\Sigma(\vartheta ; t)$ satisfies $H 1, H 2$, and $H 3$, for all $t \in[0, T], 0 \leq \vartheta \leq 1$, with the same bounds $C_{0}, \mu$ and

$$
\sup _{0 \leq \vartheta \leq 1,0 \leq t \leq T}\left(\left\|\xi_{\alpha}-e_{\alpha}\right\|_{s-1}+\left\|\xi_{\beta}-e_{\beta}\right\|_{s-1}\right) \leq M(s)<\infty,
$$

and assume that $\left(I+\widetilde{\mathcal{K}^{*}}\right)^{-1}$ is bounded from $L_{2}\left(R^{2}\right)$ to $L_{2}\left(R^{2}\right)$, with

$$
\sup _{0 \leq \vartheta \leq 1,0 \leq t \leq T}\left\|\left(I+\widetilde{\mathcal{K}^{*}}\right)^{-1}\right\| \leq \widetilde{M_{1}}<\infty,
$$

where $\left\|\left(I+\widetilde{\mathcal{K}^{*}}\right)^{-1}\right\|$ is the operator norm of $\left(I+\widetilde{\mathcal{K}^{*}}\right)^{-1}$ from $L_{2}\left(R^{2}\right)$ to $L_{2}\left(R^{2}\right)$. Assume further that for $i=1,2, \mathfrak{u}^{i}, \mathfrak{u}_{t}^{i} \in C\left([0, T], H^{s}\left(R^{2}, \mathcal{C}\left(V_{3}\right)\right)\right)$, with

$$
\sup _{0 \leq t \leq T}\left\|\mathfrak{u}^{i}\right\|_{s} \leq \mathcal{M}(s) \quad \text { and } \sup _{0 \leq t \leq T}\left\|\mathfrak{u}_{t}^{i}\right\|_{s} \leq \mathcal{M}_{t}(s), \quad i=1,2,
$$

and $\left|\mathfrak{u}_{t}^{i}+e_{3}\right| \geq c_{0}$, for some constant $c_{0}>0$. Then

$$
\begin{aligned}
\left\|\mathfrak{g}\left(\xi^{1}, \mathfrak{u}^{1}, \mathfrak{u}_{t}^{1}\right)-\mathfrak{g}\left(\xi^{2}, \mathfrak{u}^{2}, \mathfrak{u}_{t}^{2}\right)\right\|_{s} & \leq K_{g}\left(\left\|\xi^{1}-\xi^{2}\right\|_{s}+\left\|\mathfrak{u}^{1}-\mathfrak{u}^{2}\right\|_{s}+\left\|\mathfrak{u}_{t}^{1}-\mathfrak{u}_{t}^{2}\right\|_{s}\right), \\
\left\|\mathfrak{a}\left(\mathfrak{u}_{t}^{1}\right)-\mathfrak{a}\left(\mathfrak{u}_{t}^{2}\right)\right\|_{s} & \leq K_{a}\left\|\mathfrak{u}_{t}^{1}-\mathfrak{u}_{t}^{2}\right\|_{s}
\end{aligned}
$$

where $K_{g}=K_{g}\left(C_{0}, c_{0}, \mu, M(s), \widetilde{M}_{1}, \mathcal{M}(s), \mathcal{M}_{t}(s)\right)$ and $K_{a}=K_{a}\left(\mathcal{M}_{t}(s)\right)$ are constants.

Now we are ready to solve the initial value problem of the quasilinear system (5.21)-(5.23), (5.29).

Let $\Sigma(0): \xi=\xi_{0}(\alpha, \beta)$ be a surface that divides $R^{3}$ into two simply connected regions. Assume that there are vectors $e_{\alpha}, e_{\beta}$, such that $e_{\alpha} \times e_{\beta}=e_{3}, \xi_{0, \alpha}-$ $e_{\alpha}, \xi_{0, \beta}-e_{\beta} \in H^{s-1 / 2}\left(R^{2}, \mathcal{C}\left(V_{3}\right)\right)$,

$$
\begin{aligned}
& \left|\xi_{0, \alpha} \times \xi_{0, \beta}\right| \geq 2 \mu, \quad \text { for }-\infty<\alpha, \beta<\infty, \\
& \left|\xi_{0}(\alpha, \beta)-\xi_{0}\left(\alpha^{\prime}, \beta^{\prime}\right)\right| \geq 2 C_{0}\left(\left|\alpha-\alpha^{\prime}\right|+\left|\beta-\beta^{\prime}\right|\right) \quad \text { for }-\infty<\alpha, \beta<\infty .
\end{aligned}
$$

Assume $\mathfrak{u}_{0}, \mathfrak{u}_{1}$ satisfy $(5.30)$. Therefore $\tilde{\mathbf{n}}(0)=\mathbf{n}_{0}$ and $\widetilde{\mathcal{K}}^{*}(0)=\mathcal{K}_{0}^{*}$. Let

$$
M_{0}=\left\|\xi_{0, \alpha}-e_{\alpha}\right\|_{s-1 / 2}+\left\|\xi_{0, \beta}-e_{\beta}\right\|_{s-1 / 2} \quad \text { and } M_{0,1}=\left\|\left(I+\mathcal{K}_{0}^{*}\right)^{-1}\right\| .
$$


Theorem 6.19. Let $s \geq 5 / 2+q$, where $q>1$. Assume that $\mathfrak{u}_{0} \in H^{s+1 / 2}\left(R^{2}, \mathcal{C}\left(V_{3}\right)\right)$, $\mathfrak{u}_{1} \in H^{s}\left(R^{2}, \mathcal{C}\left(V_{3}\right)\right)$, and $\mathfrak{a}_{0}=\left|\mathfrak{u}_{1}+e_{3}\right| \geq 2 c_{0}$ for some constant $c_{0}>0$. Then there exists $T>0$,

$$
T=T\left(C_{0}, c_{0}, \mu, M_{0}, M_{0,1}, \mathcal{M}_{0}\right),
$$

where $\mathcal{M}_{0}^{2}=\left\|\mathfrak{u}_{0}\right\|_{s+1 / 2}^{2}+\left\|\mathfrak{u}_{1}\right\|_{s}^{2}$, such that the system (5.21)-(5.23), (5.29) has a unique solution $\mathfrak{u} \in C^{j}\left([0, T], H^{s+1 / 2-j / 2}\left(R^{2}, \mathcal{C}\left(V_{3}\right)\right)\right), j=0,1,2$. Moreover, the surfaces

$$
\Sigma(t): \xi(t)=\xi_{0}+\int_{0}^{t} \mathfrak{u}(\tau) d \tau, \quad t \in[0, T]
$$

satisfy H1-H3.

Remark 1. In this theorem, the assumption that $\mathfrak{u}_{0}, \mathfrak{u}_{1}$ satisfy (5.30) is not necessary. The result holds for general $\mathfrak{u}_{0}$ and $\mathfrak{u}_{1}$, if we assume further that $\left(I+\widetilde{\mathcal{K}}^{*}(0)\right)^{-1}$ is bounded from $L_{2}\left(R^{2}\right)$ to $L_{2}\left(R^{2}\right)$. In this case, the existence time $T$ also depends on the operator norm of $\left(I+\widetilde{\mathcal{K}}^{*}(0)\right)^{-1}$.

Remark 2. The assumption $\mathfrak{u}_{1} \in H^{s}$ is a consequence of $\mathbf{n}_{0}-e_{3} \in H^{s}$ and the regularity assumptions on $\xi_{0}, \mathfrak{u}_{0}$ given in this theorem.

Proof. Existence: We prove the existence by the iteration method.

Let $0<T<\infty$; we define a set $S_{T} \subset \bigcap_{j=0}^{2} C^{j}\left([0, T], H^{s+1 / 2-j / 2}\left(R^{2}, \mathcal{C}\left(V_{3}\right)\right)\right)$ by

$$
S_{T}=\left\{\mathfrak{u} \mid \begin{array}{l}
\sup _{0 \leq t \leq T}\left(\|\mathfrak{u}\|_{s}^{2}+\left\|\mathfrak{u}_{t}\right\|_{s-1 / 2}^{2}\right) \leq 4 \mathcal{M}_{0}^{2}, \\
\sup _{0 \leq t \leq T}\left(\|\mathfrak{u}\|_{s+1 / 2}^{2}+\left\|\mathfrak{u}_{t}\right\|_{s}^{2}\right) \leq 4 \kappa_{0}^{2} \mathcal{M}_{0}^{2}, \\
\inf _{0 \leq t \leq T} \mathfrak{a}=\inf _{0 \leq t \leq T}\left|\mathfrak{u}_{t}+e_{3}\right| \geq c_{0}
\end{array}\right\}
$$

where $\kappa_{0}=\kappa\left(C_{0}, c_{0}, \mu, 2 M_{0}, 2 M_{0,1}, K_{a}\left(2 \mathcal{M}_{0}\right)\right)$ is the constant defined in (6.24) and $K_{a}\left(2 \mathcal{M}_{0}\right)$ is the constant in Lemma $6.17 b$. For

$$
\xi(t)=\xi_{0}+\int_{0}^{t} \mathfrak{u}(\tau) d \tau
$$

where $\mathfrak{u} \in S_{T_{0}}$, for some $T_{0}>0$. Since

$$
\begin{gathered}
\left|\xi_{\alpha} \times \xi_{\beta}\right| \geq\left|\xi_{0, \alpha} \times \xi_{0, \beta}\right|-\left|\left(\int_{0}^{t} \mathfrak{u}_{\alpha}(\tau) d \tau\right) \times \xi_{0, \beta}\right| \\
-\left|\xi_{0, \alpha} \times\left(\int_{0}^{t} \mathfrak{u}_{\beta}(\tau) d \tau\right)\right|-\left|\left(\int_{0}^{t} \mathfrak{u}_{\alpha}(\tau) d \tau\right) \times\left(\int_{0}^{t} \mathfrak{u}_{\beta}(\tau) d \tau\right)\right|, \\
\left|\xi(\alpha, \beta, t)-\xi\left(\alpha^{\prime}, \beta^{\prime}, t\right)\right| \geq\left|\xi_{0}(\alpha, \beta)-\xi_{0}\left(\alpha^{\prime}, \beta^{\prime}\right)\right|-\int_{0}^{t}\left|\mathfrak{u}(\alpha, \beta, \tau)-\mathfrak{u}\left(\alpha^{\prime}, \beta^{\prime}, \tau\right)\right| d \tau, \\
\left\|\xi_{\alpha}-e_{\alpha}\right\|_{s-\frac{1}{2}}+\left\|\xi_{\beta}-e_{\beta}\right\|_{s-\frac{1}{2}} \leq\left\|\xi_{0, \alpha}-e_{\alpha}\right\|_{s-\frac{1}{2}}+\left\|\xi_{0, \beta}-e_{\beta}\right\|_{s-\frac{1}{2}} \\
\quad+\int_{0}^{t}\left(\left\|\mathfrak{u}_{\alpha}(\tau)\right\|_{s-\frac{1}{2}}+\left\|\mathfrak{u}_{\beta}(\tau)\right\|_{s-\frac{1}{2}}\right) d \tau, \\
\widetilde{\mathcal{K}^{*}}=\mathcal{K}_{0}^{*}+\int_{0}^{t}\left[\partial_{\tau}, \widetilde{\left.\mathcal{K}^{*}\right]} d \tau, \quad \mathcal{K}^{*}=\mathcal{K}_{0}^{*}+\int_{0}^{t}\left[\partial_{\tau}, \mathcal{K}^{*}\right] d \tau,\right.
\end{gathered}
$$


from Lemmas 6.1, 6.2 and 6.4, we know that there exists a

$$
T_{1}=T_{1}\left(C_{0}, \mu, M_{0}, M_{0,1}, 2 \kappa_{0}^{2} \mathcal{M}_{0}^{2}\right), \quad 0<T_{1} \leq T_{0},
$$

such that

$$
\begin{aligned}
& \inf _{0 \leq t \leq T_{1}}\left|\xi_{\alpha} \times \xi_{\beta}\right| \geq \mu, \\
& \inf _{0 \leq t \leq T_{1}}\left|\xi(\alpha, \beta, t)-\xi\left(\alpha^{\prime}, \beta^{\prime}, t\right)\right| \geq C_{0}\left(\left|\alpha-\alpha^{\prime}\right|+\left|\beta-\beta^{\prime}\right|\right), \\
& \sup _{0 \leq t \leq T_{1}}\left\|\xi_{\alpha}-e_{\alpha}\right\|_{s-\frac{1}{2}}+\left\|\xi_{\beta}-e_{\beta}\right\|_{s-\frac{1}{2}} \leq 2 M_{0}, \\
& \sup _{0 \leq t \leq T_{1}}\left\|\left(I+\widetilde{\mathcal{K}^{*}}\right)^{-1}\right\| \leq 2 M_{0,1}, \sup _{0 \leq t \leq T_{1}}\left\|\left(I+\mathcal{K}^{*}\right)^{-1}\right\| \leq 2 M_{0,1} .
\end{aligned}
$$

Step 1. We construct inductively a sequence $\mathfrak{u}^{j} \in S_{T}$, where $0<T \leq T_{1}$ will be given later. First, we take $\mathfrak{u}^{0}(t) \equiv \mathfrak{u}_{0}$ and $\xi^{0}(t) \equiv \xi_{0}$. It is clear that $\mathfrak{u}^{0} \in S_{T}$, for any $0<T<\infty$ and $\mathfrak{u}_{t t}^{0}=0 \in C\left([0, T], H^{1+q}\right)$. Assume that we have obtained $\mathfrak{u}^{m}, 0 \leq m \leq j$, and $\mathfrak{u}^{m} \in S_{T}$ for some $0<T \leq T_{1}$ and $\mathfrak{u}_{t t}^{m} \in$ $C\left([0, T], H^{1+q}\right)$. We define $\mathfrak{a}_{0}=\left|\mathfrak{u}_{1}+e_{3}\right|, \mathfrak{g}_{0}=\mathfrak{g}\left(\xi_{0}, \mathfrak{u}_{0}, \mathfrak{u}_{1}\right)$, and for $1 \leq m \leq j$, $\mathfrak{a}_{m}=\left|\mathfrak{u}_{t}^{m}+e_{3}\right|, \xi^{m}(t)=\xi_{0}+\int_{0}^{t} \mathfrak{u}^{m}(\tau) d \tau$, and $\mathfrak{g}_{m}=\mathfrak{g}\left(\xi^{m}, \mathfrak{u}^{m}, \mathfrak{u}_{t}^{m}\right)$. We know $\mathfrak{a}_{m} \geq$ $c_{0}$, for $0 \leq m \leq j$. From Lemma 6.17 , we have that $\mathfrak{a}_{m}-1 \in C\left([0, T], H^{s}\left(R^{2}\right)\right)$, $\mathfrak{g}_{m} \in C\left([0, T], H^{s}\left(R^{2}, \mathcal{C}\left(V_{3}\right)\right)\right)$, and $\partial_{t} \mathfrak{a}_{m} \in C\left([0, T], H^{1+q}\right)$. Therefore by Theorem 6.16 , there is a unique $\mathfrak{u}^{j+1} \in \bigcap_{j=0}^{2} C\left([0, T], H^{s+1 / 2-j / 2}\left(R^{2}, \mathcal{C}\left(V_{3}\right)\right)\right)$, satisfying the following equation:

$$
\begin{aligned}
& \mathfrak{u}_{t t}^{j+1}+\mathfrak{a}_{j} \nabla_{\mathbf{n}_{j}} \mathfrak{u}^{j+1}=\mathfrak{g}_{j}, \\
& \mathfrak{u}^{j+1}(\cdot, 0)=\mathfrak{u}_{0}, \quad \mathfrak{u}_{t}^{j+1}(\cdot, 0)=\mathfrak{u}_{1}
\end{aligned}
$$

where $\nabla_{\mathbf{n}_{j}}$ is the normal derivative with respect to the surface $\xi^{j}(t)$ for fixed $t$, $0 \leq t \leq T$. We want to show that there is a $0<T \leq T_{1}$, such that $\mathfrak{u}^{m} \in S_{T}$ for $0 \leq m \leq j$ implies $\mathfrak{u}^{j+1} \in S_{T}$.

Assume that $\mathfrak{u}^{j} \in S_{T}$, where $0<T \leq T_{1}$. From (6.43), Lemma 6.14 (6.37) and Lemma 6.17, we have

$$
\left|\left(\mathfrak{u}^{j+1}(t), \mathfrak{u}_{t}^{j+1}(t)\right)\right|_{s}^{2} \leq \kappa_{0}^{2} e^{d_{1} t}\left|\left(\mathfrak{u}_{0}, \mathfrak{u}_{1}\right)\right|_{s}^{2}+\kappa_{0} \int_{0}^{t} e^{d_{1}(t-\tau)}\left\|\mathfrak{g}_{j}(\tau)\right\|_{s}^{2} d \tau,
$$

where $\kappa_{0}=\kappa\left(C_{0}, c_{0}, \mu, 2 M_{0}, 2 M_{0,1}, K_{a}\left(2 \mathcal{M}_{0}\right)\right)$ is the constant in (6.42),

$$
d_{1}=d_{1}\left(C_{0}, c_{0}, \mu, 2 M_{0}, 2 M_{0,1}, K_{a}\left(2 \kappa_{0} \mathcal{M}_{0}\right), K_{a_{t}}, 2 \kappa_{0} \mathcal{M}_{0}\right),
$$

is the constant defined in inequality (6.37), and $K_{a_{t}}$ is the constant such that $\sup _{0 \leq t \leq T}\left\|\partial_{t} \mathfrak{a}_{j}\right\|_{1+q} \leq K_{a_{t}}$. From Lemma $6.17 c$, we know that

$$
K_{a_{t}}=K_{a_{t}}\left(2 \mathcal{M}_{0}, \mathcal{M}_{t t}\right)
$$

where $\sup _{0 \leq t \leq T}\left\|\mathfrak{u}_{t t}^{j}\right\|_{1+q} \leq \mathcal{M}_{t t}$. Since from (6.44), $\mathfrak{u}_{t t}^{j}=-\mathfrak{a}_{j-1} \nabla_{\mathbf{n}_{j-1}} u^{j}+\mathfrak{g}_{j-1}$, therefore

$$
\sup _{0 \leq t \leq T}\left\|\mathfrak{u}_{t t}^{j}\right\|_{1+q} \leq \mathcal{M}_{t t}\left(\mathcal{M}_{0}, M_{0,1}, C_{0}, c_{0}, \mu, M_{0}\right)
$$

where $M_{t t}\left(\mathcal{M}_{0}, M_{0,1}, C_{0}, c_{0}, \mu, M_{0}\right)$ is a constant determined by $\mathcal{M}_{0}, M_{0,1}, C_{0}, c_{0}$, $\mu, M_{0}$. So there exists a constant

$$
K_{1}=K_{1}\left(C_{0}, c_{0}, \mu, M_{0}, M_{0,1}, \mathcal{M}_{0}\right),
$$


such that

$$
\sup _{0 \leq t \leq T \leq T_{1}}\left|\left(\mathfrak{u}^{j+1}(t), \mathfrak{u}_{t}^{j+1}(t)\right)\right|_{s}^{2} \leq K_{1}
$$

Since

$$
\begin{aligned}
\left\|\mathfrak{u}^{j+1}(t)\right\|_{s}^{2}+\left\|\mathfrak{u}_{t}^{j+1}(t)\right\|_{s-1 / 2}^{2} \leq & 2\left\|\mathfrak{u}_{0}\right\|_{s}^{2}+2\left\|\mathfrak{u}_{1}\right\|_{s-1 / 2}^{2} \\
& +2 \int_{0}^{t}\left(\left\|\mathfrak{u}_{t}^{j+1}(\tau)\right\|_{s}^{2}+\left\|\mathfrak{u}_{t t}^{j+1}(\tau)\right\|_{s-1 / 2}^{2}\right) d \tau
\end{aligned}
$$

for $t \leq 1$ and from (6.44), Lemma 6.17, and the induction hypothesis, there is a constant

$$
K_{2}=K_{2}\left(C_{0}, c_{0}, \mu, 2 M_{0}, 2 M_{0,1}, K_{a}\left(2 \kappa_{0} \mathcal{M}_{0}\right), 2 \kappa_{0} \mathcal{M}_{0}\right)
$$

such that

$$
\left\|\mathfrak{u}_{t}^{j+1}(\tau)\right\|_{s}^{2}+\left\|\mathfrak{u}_{t t}^{j+1}(\tau)\right\|_{s-1 / 2}^{2} \leq K_{2}\left(\left|\left(\mathfrak{u}^{j+1}(\tau), \mathfrak{u}_{t}^{j+1}(\tau)\right)\right|_{s}^{2}+1\right),
$$

it follows that for all $0<T \leq \min \left\{T_{1}, T_{2}, 1\right\}$, where $T_{2}=\frac{\mathcal{M}_{0}^{2}}{\left(K_{1}+1\right) K_{2}}$, we have

$$
\sup _{0 \leq t \leq T}\left(\left\|\mathfrak{u}^{j+1}(t)\right\|_{s}^{2}+\left\|\mathfrak{u}_{t}^{j+1}(t)\right\|_{s-1 / 2}^{2}\right) \leq 2 \mathcal{M}_{0}^{2}+T K_{2}\left(K_{1}+1\right) \leq 4 \mathcal{M}_{0}^{2}
$$

Now assume that $0<T \leq \min \left\{T_{1}, T_{2}, 1\right\}$. From the induction hypothesis, Lemma 6.14 (6.38), Lemma 6.17 and (6.43), we also have that

$$
\left|\left(\mathfrak{u}^{j+1}(t), \mathfrak{u}_{t}^{j+1}(t)\right)\right|_{s}^{2} \leq \kappa_{0}^{2} e^{d_{2}^{\prime} t}\left|\left(\mathfrak{u}_{0}, \mathfrak{u}_{1}\right)\right|_{s}^{2}
$$

where $\kappa_{0}$ is as in (6.42),

$$
+\kappa_{0} \int_{0}^{t} e^{d_{2}^{\prime}(t-\tau)}\left(\left\|\mathfrak{a}_{j}-1\right\|_{s}^{2}+\left\|\xi_{t}^{j}\right\|_{s+1 / 2}^{2}+\left\|\mathfrak{g}_{j}(\tau)\right\|_{s}^{2}\right) d \tau
$$

$$
d_{2}^{\prime}=d_{2}\left(C_{0}, c_{0}, \mu, 2 M_{0}, 2 M_{0,1}, K_{a}\left(2 \mathcal{M}_{0}\right), K_{a_{t}}, 2 \mathcal{M}_{0}, \sup _{0 \leq t \leq T}\left\|\mathfrak{u}^{j+1}(t)\right\|_{1+q}\right)
$$

is the constant defined in (6.38), and $K_{a_{t}}$ is as in (6.45). From (6.47) we have that

$$
\sup _{0 \leq t \leq T}\left\|\mathfrak{u}^{j+1}(t)\right\|_{1+q} \leq 2 \mathcal{M}_{0}
$$

therefore

$$
d_{2}^{\prime} \leq d_{2}=d_{2}\left(C_{0}, c_{0}, \mu, 2 M_{0}, 2 M_{0,1}, K_{a}\left(2 \mathcal{M}_{0}\right), K_{a_{t}}, 2 \mathcal{M}_{0}, 2 \mathcal{M}_{0}\right) .
$$

Moreover we have from Lemma 6.17 and the induction hypothesis that

$$
\left\|\mathfrak{a}_{j}-1\right\|_{s} \leq K_{a}\left(2 \kappa_{0} \mathcal{M}_{0}\right), \quad\left\|\xi_{t}^{j}\right\|_{s+1 / 2}=\left\|\mathfrak{u}^{j}\right\|_{s+1 / 2} \leq 2 \kappa_{0} \mathcal{M}_{0}, \quad\left\|\mathfrak{g}_{j}\right\|_{s} \leq K_{g},
$$

where $K_{g}=K_{g}\left(C_{0}, c_{0}, \mu, 2 M_{0}, 2 M_{0,1}, 2 \mathcal{M}_{0}, 2 \kappa_{0} \mathcal{M}_{0}\right)$ is the constant determined in Lemma 6.17 a. Therefore

$$
\begin{aligned}
\left|\left(\mathfrak{u}^{j+1}(t), \mathfrak{u}_{t}^{j+1}(t)\right)\right|_{s}^{2} \leq & \kappa_{0}^{2} e^{d_{2} t}\left|\left(\mathfrak{u}_{0}, \mathfrak{u}_{1}\right)\right|_{s}^{2} \\
& +\kappa_{0} \int_{0}^{t} e^{d_{2}(t-\tau)}\left(K_{a}^{2}\left(2 \kappa_{0} \mathcal{M}_{0}\right)+4 \kappa_{0}^{2} \mathcal{M}_{0}^{2}+K_{g}^{2}\right) d \tau \\
\leq & \kappa_{0}^{2} \mathcal{M}_{0}^{2} e^{d_{2} t}+K_{3} t e^{d_{2} t}
\end{aligned}
$$

where

$$
K_{3}=\kappa_{0}\left(K_{a}^{2}\left(2 \kappa_{0} \mathcal{M}_{0}\right)+4 \kappa_{0}^{2} \mathcal{M}_{0}^{2}+K_{g}^{2}\right) .
$$


Taking $T_{3}=\min \left\{\frac{\ln 2}{d_{2}}, \frac{\kappa_{0}^{2} \mathcal{M}_{0}^{2}}{K_{3}}\right\}$, we have for all $0<T \leq \min \left\{T_{1}, T_{2}, T_{3}, 1\right\}$,

$$
\sup _{0 \leq t \leq T}\left|\left(\mathfrak{u}^{j+1}(t), \mathfrak{u}_{t}^{j+1}(t)\right)\right|_{s}^{2} \leq 4 \kappa_{0}^{2} \mathcal{M}_{0}^{2} .
$$

We now show that there is a $0<T \leq \min \left\{T_{1}, T_{2}, T_{3}, 1\right\}$, such that

$$
\inf _{0 \leq t \leq T} \mathfrak{a}_{j+1}(t)=\inf _{0 \leq t \leq T}\left|\mathfrak{u}_{t}^{j+1}(t)+e_{3}\right| \geq c_{0} .
$$

Assume that $0<T \leq \min \left\{T_{1}, T_{2}, T_{3}, 1\right\}$. By the same argument of $(6.45)$, we have

$$
\sup _{0 \leq t \leq T}\left\|\partial_{t} \mathfrak{a}_{j+1}\right\|_{1+q} \leq K_{a_{t}},
$$

where $K_{a_{t}}$ is as defined in (6.45). Therefore

$$
\sup _{0 \leq t \leq T}\left|\partial_{t} \mathfrak{a}_{j+1}\right|_{\infty} \leq k_{0} K_{a_{t}}
$$

where $k_{0}$ is the constant from Lemma 6.1.

Taking $T_{4}=\frac{c_{0}}{k_{0} K_{a_{t}}}$ and $T=\min \left\{T_{1}, T_{2}, T_{3}, T_{4}, 1\right\}$, we have for $0 \leq t \leq T$,

$$
\mathfrak{a}_{j+1}(t)=\left|\mathfrak{u}_{t}^{j+1}(t)+1\right| \geq \mathfrak{a}_{0}-\int_{0}^{t}\left|\partial_{\tau} \mathfrak{a}_{j+1}(\tau)\right|_{\infty} d \tau \geq 2 c_{0}-t k_{0} K_{a_{t}} \geq c_{0} .
$$

So we have obtained a $T>0, T=\min \left\{T_{1}, T_{2}, T_{3}, T_{4}, 1\right\}=T\left(C_{0}, c_{0}, \mu, M_{0}, M_{0,1}, \mathcal{M}_{0}\right)$ and a sequence $\mathfrak{u}^{j} \in S_{T}, j=0,1,2, \ldots$, such that $\mathfrak{u}^{j}$ satisfies the recursive equation (6.44).

Step 2. We want to show that the sequence $\mathfrak{u}^{j} \in S_{T}$ constructed in Step 1 is a Cauchy sequence in some Banach space. Let $\hat{\mathfrak{u}}=\mathfrak{u}^{j+1}-\mathfrak{u}^{j}$. Then $\hat{\mathfrak{u}} \in$ $\bigcap_{j=0}^{2} C^{j}\left([0, T], H^{s+1 / 2-j / 2}\left(R^{2}, \mathcal{C}\left(V_{3}\right)\right)\right)$, and $\hat{\mathfrak{u}}$ satisfies

$$
\begin{aligned}
& \hat{\mathfrak{u}}_{t t}+\mathfrak{a}_{j} \nabla_{\mathbf{n}_{j}} \hat{\mathfrak{u}}=\overline{\mathfrak{g}}, \\
& \hat{\mathfrak{u}}(\cdot, 0)=0, \quad \hat{\mathfrak{u}}_{t}(\cdot, 0)=0
\end{aligned}
$$

where $\overline{\mathfrak{g}}=\mathfrak{g}_{j}-\mathfrak{g}_{j-1}-\left(\mathfrak{a}_{j}-\mathfrak{a}_{j-1}\right) \nabla_{\mathbf{n}_{j}} \mathfrak{u}^{j}-\mathfrak{a}_{j-1}\left(\nabla_{\mathbf{n}_{j}}-\nabla_{\mathbf{n}_{j-1}}\right) \mathfrak{u}^{j}$. From Lemmas 6.18 and 6.11 , we know that there is a constant $K_{4}=K_{4}\left(C_{0}, c_{0}, \mu, M_{0}, M_{0,1}, \mathcal{M}_{0}\right)$, such that

$$
\begin{aligned}
\|\overline{\mathfrak{g}}(t)\|_{s-1 / 2} \leq & K_{4}\left(\left\|\xi^{j-1}(t)-\xi^{j}(t)\right\|_{s-1 / 2}\right. \\
& \left.+\left\|\mathfrak{u}^{j-1}(t)-\mathfrak{u}^{j}(t)\right\|_{s-1 / 2}+\left\|\mathfrak{u}_{t}^{j-1}(t)-\mathfrak{u}_{t}^{j}(t)\right\|_{s-1 / 2}\right) .
\end{aligned}
$$

Therefore using Lemma 6.14 (6.37), we get

$$
\begin{aligned}
\left|\left(\hat{\mathfrak{u}}, \hat{\mathfrak{u}}_{t}\right)\right|_{s-1 / 2}^{2} & \leq \kappa_{0} \int_{0}^{t} e^{d_{1}(t-\tau)}\|\overline{\mathfrak{g}}(\tau)\|_{s-1 / 2}^{2} d \tau \\
& \leq 4 \kappa_{0} K_{4}^{2} e^{d_{1} t} \int_{0}^{t}\left\|\xi^{j-1}(\tau)-\xi^{j}(\tau)\right\|_{s-1 / 2}^{2} d \tau \\
& +4 \kappa_{0} K_{4}^{2} e^{d_{1} t} \int_{0}^{t}\left(\left\|\mathfrak{u}^{j-1}(\tau)-\mathfrak{u}^{j}(\tau)\right\|_{s-1 / 2}^{2}+\left\|\mathfrak{u}_{t}^{j-1}(\tau)-\mathfrak{u}_{t}^{j}(\tau)\right\|_{s-1 / 2}^{2}\right) d \tau
\end{aligned}
$$

where $d_{1}=d_{1}\left(C_{0}, c_{0}, \mu, 2 M_{0}, 2 M_{0,1}, K_{a}\left(2 \kappa_{0} \mathcal{M}_{0}\right), K_{a_{t}}, 2 \kappa_{0} \mathcal{M}_{0}\right)$ is the constant defined in (6.37) and $\kappa_{0}$ is the constant in (6.42). Since

$$
\xi^{j-1}(t)-\xi^{j}(t)=\int_{0}^{t}\left(\mathfrak{u}^{j-1}(\tau)-\mathfrak{u}^{j}(\tau)\right) d \tau
$$


we have

$$
\left\|\xi^{j-1}(t)-\xi^{j}(t)\right\|_{s-1 / 2} \leq \int_{0}^{t}\left\|\mathfrak{u}^{j-1}(\tau)-\mathfrak{u}^{j}(\tau)\right\|_{s-1 / 2} d \tau
$$

therefore

$$
\int_{0}^{t}\left\|\xi^{j-1}(\tau)-\xi^{j}(\tau)\right\|_{s-1 / 2}^{2} d \tau \leq t^{2} \int_{0}^{t}\left\|\mathfrak{u}^{j-1}(\tau)-\mathfrak{u}^{j}(\tau)\right\|_{s-1 / 2}^{2} d \tau
$$

and

$$
\begin{aligned}
& \left|\left(\hat{\mathfrak{u}}, \hat{\mathfrak{u}}_{t}\right)\right|_{s-1 / 2}^{2}=\left|\left(\mathfrak{u}^{j+1}(t)-\mathfrak{u}^{j}(t), \mathfrak{u}_{t}^{j+1}(t)-\mathfrak{u}_{t}^{j}(t)\right)\right|_{s-1 / 2}^{2} \\
& \quad \leq 4 \kappa_{0} K_{4}^{2} e^{d_{1} t}\left(t^{2}+1\right) \int_{0}^{t}\left(\left\|\mathfrak{u}^{j-1}(\tau)-\mathfrak{u}^{j}(\tau)\right\|_{s-1 / 2}^{2}+\left\|\mathfrak{u}_{t}^{j-1}(\tau)-\mathfrak{u}_{t}^{j}(\tau)\right\|_{s-1 / 2}^{2}\right) d \tau \\
& \quad \leq 4 \kappa_{0} K_{4}^{2} e^{d_{1} T}\left(T^{2}+1\right) \int_{0}^{t}\left|\left(\mathfrak{u}^{j-1}(\tau)-\mathfrak{u}^{j}(\tau), \mathfrak{u}_{t}^{j-1}(\tau)-\mathfrak{u}_{t}^{j}(\tau)\right)\right|_{s-1 / 2}^{2} d \tau .
\end{aligned}
$$

This proves that $\mathfrak{u}^{j}$ is a Cauchy sequence in $\bigcap_{j=0}^{1} C^{j}\left([0, T], H^{s-j / 2}\left(R^{2}, \mathcal{C}\left(V_{3}\right)\right)\right)$. Therefore there exists $\mathfrak{u} \in \bigcap_{j=0}^{1} C^{j}\left([0, T], H^{s-j / 2}\left(R^{2}, \mathcal{C}\left(V_{3}\right)\right)\right)$, such that

$$
\mathfrak{u}^{j} \rightarrow \mathfrak{u} \quad \text { in } \bigcap_{j=0}^{1} C^{j}\left([0, T], H^{s-j / 2}\left(R^{2}, \mathcal{C}\left(V_{3}\right)\right)\right) .
$$

Furthermore, $\mathfrak{a}_{j} \rightarrow \mathfrak{a}=\left|\mathfrak{u}_{t}+e_{3}\right|$ and $\mathfrak{g}_{j} \rightarrow \mathfrak{g}\left(\xi, \mathfrak{u}, \mathfrak{u}_{t}\right)$ in $C\left([0, T], H^{s-1 / 2}\right)$, where $\xi=\xi_{0}+\int_{0}^{t} \mathfrak{u} d \tau$. Let $j \rightarrow \infty$; from (6.44) we get $\mathfrak{u}$ is a solution of system (5.21)(5.23),(5.29), and from (6.43),

$$
\xi(t)=\xi_{0}+\int_{0}^{t} \mathfrak{u}(\tau) d \tau, \quad 0 \leq t \leq T,
$$

satisfies H1-H3. We want to further verify that

$$
\mathfrak{u} \in \bigcap_{j=0}^{2} C^{j}\left([0, T], H^{s+1 / 2-j / 2}\left(R^{2}, \mathcal{C}\left(V_{3}\right)\right)\right) .
$$

Since $\mathfrak{u}^{j} \in S_{T}$, we have that

$$
\mathfrak{u} \in L^{\infty}\left([0, T], H^{s+1 / 2}\left(R^{2}, \mathcal{C}\left(V_{3}\right)\right)\right) \quad \text { and } \quad \mathfrak{u}_{t} \in L^{\infty}\left([0, T], H^{s}\left(R^{2}, \mathcal{C}\left(V_{3}\right)\right)\right) .
$$

Using Lemma 6.15 and a similar argument as that of Theorem 5.11 in [24], we get

$$
\mathfrak{u} \in \bigcap_{j=0}^{2} C^{j}\left([0, T], H^{s+1 / 2-j / 2}\left(R^{2}, \mathcal{C}\left(V_{3}\right)\right)\right) .
$$

This proves the existence.

Uniqueness: The uniqueness can be proved by a similar argument as in step 2 . We omit it. 


\section{§7. THE NONLINEAR WATER WAVE EQUATION}

Assume that the initial surface $\Sigma(0): \xi=\xi_{0}(\alpha, \beta)$ and initial velocity $\mathfrak{u}_{0}$ are as given in the initial data part of $\S 5$, and that the initial acceleration $\mathfrak{u}_{1}$ satisfies (5.30). Assume further that $\xi_{0}, \mathfrak{u}_{0}$ and $\mathfrak{u}_{1}$ are in the Sobolev spaces as given in Theorem 6.19. In this section, we show that a solution of the quasilinear system (5.21)-(5.23), (5.29) is also a solution of the water wave system $(4.3),(4.4),\left(4.5^{\prime}\right)$.

Let $\mathfrak{u}$ be the solution obtained in Theorem 6.19, and let $\Sigma(t): \xi=\xi_{0}(\alpha, \beta)+$ $\int_{0}^{t} \mathfrak{u}(\alpha, \beta, \tau) d \tau$, for $t \in[0, T]$, where $T$ is the time obtained in Theorem 6.19. Assume $\Omega(t)$ and $\Omega(t)^{c}$ are the lower and upper regions with common boundary $\Sigma(t), N=\xi_{\alpha} \times \xi_{\beta}$, and $\mathbf{n}=\frac{N}{|N|}$. In order to prove that $\Sigma(t): \xi=$ $\xi_{0}(\alpha, \beta)+\int_{0}^{t} \mathfrak{u}(\alpha, \beta, \tau) d \tau, t \in[0, T]$, is a solution of (4.3),(4.4),(4.5'), it is enough to show $\tilde{\mathbf{n}}=\mathbf{n}$ and $\mathfrak{u}=H_{\Sigma(t)} \mathfrak{u}$ for $0 \leq t \leq T$. We know when $t=0, \tilde{\mathbf{n}}(0)=\mathbf{n}(0)$ and $\mathfrak{u}_{0}=H_{\Sigma(0)} \mathfrak{u}_{0}$.

We first introduce some notation and develop some identities and lemmas. We denote by $\nabla_{\xi}$ or $\nabla_{\xi}^{+}=\left(\partial_{x}^{+}, \partial_{y}^{+}, \partial_{z}^{+}\right)$the space valuable gradient with respect to $\Omega(t)$ as defined in (3.9), and by $\nabla_{\xi}^{-}=\left(\partial_{x}^{-}, \partial_{y}^{-}, \partial_{z}^{-}\right)$the space valuable gradient with respect to $\Omega(t)^{c} ; \nabla_{\mathbf{n}}^{ \pm}=\mathbf{n} \cdot \nabla_{\xi}^{ \pm}$are the normal derivatives as defined in (3.10), $\nabla_{\mathbf{n}}=\nabla_{\mathbf{n}}^{+} ; \mathcal{D}_{\xi}, \mathcal{D}_{\xi}^{ \pm}$are the Clifford 1-vector counterparts of $\nabla_{\xi}$ and $\nabla_{\xi}^{ \pm}$respectively; $H_{\Sigma(t)}$ is the Hilbert transform with respect to the region $\Omega(t)$ as defined in (3.1), therefore the Hilbert transform with respect to $\Omega(t)^{c}$ is $-H_{\Sigma(t)}$. For $\sigma=\sigma_{1} e_{1}+$ $\sigma_{2} e_{2}+\sigma_{3} e_{3}+\sigma_{123} e_{1} e_{2} e_{3}$, we denote by $\sigma_{(3)}$ the 3 -vector part $\sigma_{123} e_{1} e_{2} e_{3}$ of $\sigma$; $\widetilde{H}_{\Sigma(t)} \mathfrak{u}=H_{\Sigma(t)} \mathfrak{u}-\left\{H_{\Sigma(t)} \mathfrak{u}\right\}_{(3)}$ is therefore the 1-vector part of $H_{\Sigma(t)} \mathfrak{u}$. For the purpose of this section, we do not distinguish the notation for operators bounded from $L_{2}\left(R^{2}\right)$ to $L_{2}\left(R^{2}\right)$, they are indicated by either $\mathcal{T}$ or $\mathcal{T}_{i}, i=1,2,3, \ldots$

Claim 1. Let $f$ be a real scalar-valued function on $R^{2}$. Then

$$
\Re\left\{\left(I-H_{\Sigma(t)}\right)(I-\mathcal{K})^{-1} f\right\}=f,
$$

where $\mathcal{K}$ is the double layered potential operator on $\Sigma(t)$ as defined in (2.11).

Claim 1 is straightforward by definition.

Claim 2. Assume that $F=f_{1} e_{1}+f_{2} e_{2}+f_{3} e_{3}$, and $F=-H_{\Sigma(t)} F$. Then

$$
F=-\left(I-H_{\Sigma(t)}\right)\left(\mathbf{n}\left(I+\mathcal{K}^{*}\right)^{-1} \Re(\mathbf{n} F)\right) .
$$

Proof. Let $F^{\hbar}$ be the harmonic extension of $F$ on $\Omega(t)^{c}$. Then $F^{\hbar}$ is Clifford analytic on $\Omega(t)^{c}$. Therefore $\operatorname{curl} F^{\hbar}=\operatorname{div} F^{\hbar}=0$. Since $\Omega(t)^{c}$ is simply connected, there is a function $f, f$ harmonic on $\Omega(t)^{c}$, such that $F^{\hbar}=\mathcal{D}^{-} f$. (7.2) is therefore a consequence of (3.14).

Claim 3. $\nabla_{\mathbf{n}}^{+}+\nabla_{\mathbf{n}}^{-}$is a bounded operator from $L_{2}(\Sigma(t), d S)$ to $L_{2}(\Sigma(t), d S)$.

This is a standard result from potential theory; see [15, Theorem 5.2].

\section{Claim 4.}

$$
N_{t}=-\frac{1}{2}|N|\left\{\nabla_{\mathbf{n}}^{+}\left(\mathfrak{u}+\widetilde{H}_{\Sigma(t)} \mathfrak{u}\right)+\nabla_{\mathbf{n}}^{-}\left(\mathfrak{u}-\widetilde{H}_{\Sigma(t)} \mathfrak{u}\right)\right\} .
$$


Proof. A calculation as in (5.4) shows that

$$
\begin{aligned}
(\mathfrak{u} \pm & \left.\widetilde{H}_{\Sigma(t)} \mathfrak{u}\right)_{\alpha} \times \xi_{\beta}+\xi_{\alpha} \times\left(\mathfrak{u} \pm \widetilde{H}_{\Sigma(t)} \mathfrak{u}\right)_{\beta} \\
& =-\left(N \cdot \nabla_{\xi}^{ \pm}\right)\left(\mathfrak{u} \pm \widetilde{H}_{\Sigma(t)} \mathfrak{u}\right)-N \times\left(\mathcal{D}_{\xi}^{ \pm} \times\left(\mathfrak{u} \pm \widetilde{H}_{\Sigma(t)} \mathfrak{u}\right)\right) .
\end{aligned}
$$

Since $\mathfrak{u}+H_{\Sigma(t)} \mathfrak{u}$ and $\mathfrak{u}-H_{\Sigma(t)} \mathfrak{u}$ are the boundary values of Clifford analytic functions on $\Omega(t)$ and $\Omega(t)^{c}$ respectively, we have

$$
\mathcal{D}_{\xi}^{ \pm}\left(\mathfrak{u} \pm H_{\Sigma(t)} \mathfrak{u}\right)=0
$$

therefore its 2-vector part

$$
\mathcal{D}_{\xi}^{ \pm} \times\left(\mathfrak{u} \pm \widetilde{H}_{\Sigma(t)} \mathfrak{u}\right) \pm D_{\xi}^{ \pm}\left(\left\{H_{\Sigma(t)} \mathfrak{u}\right\}_{(3)}\right)=0 .
$$

So

$$
\begin{aligned}
N \times\left(\mathcal{D}_{\xi}^{ \pm} \times\left(\mathfrak{u} \pm \widetilde{H}_{\Sigma(t)} \mathfrak{u}\right)\right) & =\mp\left(\xi_{\alpha} \times \xi_{\beta}\right) \times D_{\xi}^{ \pm}\left(\left\{H_{\Sigma(t)} \mathfrak{u}\right\}_{(3)}\right) \\
& =\mp\left[\xi_{\beta} \partial_{\alpha}\left(\left\{H_{\Sigma(t)} \mathfrak{u}\right\}_{(3)}\right)-\xi_{\alpha} \partial_{\beta}\left(\left\{H_{\Sigma(t)} \mathfrak{u}\right\}_{(3)}\right)\right] .
\end{aligned}
$$

This implies that

$$
\begin{aligned}
(\mathfrak{u} \pm & \left.\widetilde{H}_{\Sigma(t)} \mathfrak{u}\right)_{\alpha} \times \xi_{\beta}+\xi_{\alpha} \times\left(\mathfrak{u} \pm \widetilde{H}_{\Sigma(t)} \mathfrak{u}\right)_{\beta} \\
& =-\left(N \cdot \nabla_{\xi}^{ \pm}\right)\left(\mathfrak{u} \pm \widetilde{H}_{\Sigma(t)} \mathfrak{u}\right) \pm\left[\xi_{\beta} \partial_{\alpha}\left(\left\{H_{\Sigma(t)} \mathfrak{u}\right\}_{(3)}\right)-\xi_{\alpha} \partial_{\beta}\left(\left\{H_{\Sigma(t)} \mathfrak{u}\right\}_{(3)}\right)\right]
\end{aligned}
$$

and

$$
\left.N_{t}=\mathfrak{u}_{\alpha} \times \xi_{\beta}+\xi_{\alpha} \times \mathfrak{u}_{\beta}=-\frac{1}{2}\left[\left(N \cdot \nabla_{\xi}^{+}\right)\left(\mathfrak{u}+\widetilde{H}_{\Sigma(t)} \mathfrak{u}\right)+\left(N \cdot \nabla_{\xi}^{-}\right)\left(\mathfrak{u}-\widetilde{H}_{\Sigma(t)} \mathfrak{u}\right)\right)\right]
$$

This proofs (7.3).

A consequence of (7.3) and Claim 3 is that

$$
\mathbf{n}_{t}=\left(\nabla_{\mathbf{n}}\left(\widetilde{H}_{\Sigma(t)} \mathfrak{u}\right) \times \mathbf{n}\right) \times \mathbf{n}+\mathcal{T}\left(\mathfrak{u}-H_{\Sigma(t)} \mathfrak{u}\right),
$$

where $\mathcal{T}$ is a bounded linear operator from $L_{2}\left(R^{2}\right)$ to $L_{2}\left(R^{2}\right)$.

Claim 5. Let $\mathfrak{u}$ be a solution of (5.21)-(5.23). Then

$$
\left\{\nabla_{\mathbf{n}}\left(H_{\Sigma(t)} \mathfrak{u}\right)\right\}_{(3)}=\left\{H_{\Sigma(t)}\left(\nabla_{\mathbf{n}} \mathfrak{u}+\mathbf{n}_{t}\right)\right\}_{(3)}+\mathcal{T}\left(\mathfrak{u}-H_{\Sigma(t)} \mathfrak{u}\right)
$$

where $\mathcal{T}$ is a bounded operator from $L_{2}$ to $L_{2}$.

Proof. Since $N \mathcal{D}_{\xi}^{ \pm}\left(\mathfrak{u} \pm H_{\Sigma(t)} \mathfrak{u}\right)=0$, we have that its 3-vector part

$$
\mp N \cdot \mathcal{D}_{\xi}^{ \pm}\left(\left\{H_{\Sigma(t)} \mathfrak{u}\right\}_{(3)}\right)-\left(N \times \mathcal{D}_{\xi}^{ \pm}\right) \cdot\left(\mathfrak{u} \pm \widetilde{H}_{\Sigma(t)} \mathfrak{u}\right) e_{1} e_{2} e_{3}=0 .
$$

From $N \times \mathcal{D}_{\xi}^{ \pm}=\xi_{\beta} \partial_{\alpha}-\xi_{\alpha} \partial_{\beta}$, we have by adding up the above two identities,

$$
\left(N \cdot \mathcal{D}_{\xi}^{+}-N \cdot \mathcal{D}_{\xi}^{-}\right)\left(\left\{H_{\Sigma(t)} \mathfrak{u}\right\}_{(3)}\right)=-2\left(N \times \mathcal{D}_{\xi}\right) \cdot \mathfrak{u} e_{1} e_{2} e_{3},
$$

so

$$
\left.\left.\nabla_{\mathbf{n}}\left(\left\{H_{\Sigma(t)} \mathfrak{u}\right)\right\}_{(3)}\right)=\frac{1}{2}\left(\nabla_{\mathbf{n}}^{+}+\nabla_{\mathbf{n}}^{-}\right)\left(\left\{H_{\Sigma(t)} \mathfrak{u}\right)\right\}_{(3)}\right)-\left(\mathbf{n} \times \mathcal{D}_{\xi}\right) \cdot \mathfrak{u} e_{1} e_{2} e_{3} .
$$

On the other hand,

$$
\mathbf{n} \mathcal{D}_{\xi} \mathfrak{u}=\mathbf{n} H_{\Sigma(t)}\left(\mathcal{D}_{\xi} \mathfrak{u}\right)=\left(\mathbf{n} H_{\Sigma(t)}+H_{\Sigma(t)} \mathbf{n}\right)\left(\mathcal{D}_{\xi} \mathfrak{u}\right)-H_{\Sigma(t)}\left(\mathbf{n} \mathcal{D}_{\xi} \mathfrak{u}\right)
$$

Taking the 3 -vector part of both sides of (7.6), we get

$$
\begin{aligned}
-\left(\mathbf{n} \times \mathcal{D}_{\xi}\right) \cdot \mathfrak{u} e_{1} e_{2} e_{3}= & \left\{\left(\mathbf{n} H_{\Sigma(t)}+H_{\Sigma(t)} \mathbf{n}\right)\left(\mathcal{D}_{\xi} \mathfrak{u}\right)\right\}_{(3)} \\
& +\left\{H_{\Sigma(t)}\left(\nabla_{\mathbf{n}} \mathfrak{u}-\left(\mathbf{n} \times \mathcal{D}_{\xi}\right) \times \mathfrak{u}\right)\right\}_{(3)} .
\end{aligned}
$$


Notice that

$$
\mathcal{D}_{\xi} \mathfrak{u}=\frac{1}{2} \mathcal{D}_{\xi}\left(\mathfrak{u}-H_{\Sigma(t)} \mathfrak{u}\right)
$$

$\left(\mathbf{n} \times \mathcal{D}_{\xi}\right) \times \mathfrak{u}=-\frac{N_{t}}{|N|}=-\mathbf{n}_{t}+\partial_{t}\left(\frac{1}{|N|}\right) N$, and the operator $\left(\mathbf{n} H_{\Sigma(t)}+H_{\Sigma(t)} \mathbf{n}\right) \mathcal{D}_{\xi}$ is a bounded operator from $L_{2}$ to $L_{2}$. Together with Claim 3, we conclude (7.5).

Claim 6. Let $\mathfrak{u}$ be a solution of (5.21)-(5.23). Then

$$
\left\{\left(H_{\Sigma(t)} \mathfrak{u}\right)_{t t}+\mathfrak{a} \nabla_{\mathbf{n}}\left(H_{\Sigma(t)} \mathfrak{u}\right)\right\}_{(3)}=\mathcal{T}_{1}\left(\mathfrak{u}-H_{\Sigma(t)} \mathfrak{u}\right)+\mathcal{T}_{2}(\tilde{\mathbf{n}}-\mathbf{n})
$$

where $\mathcal{T}_{1} \mathcal{T}_{2}$ are bounded operators from $L_{2}$ to $L_{2}$.

Proof. We have from integration by parts that

$$
\begin{aligned}
\left\{H_{\Sigma(t)} \mathfrak{u}\right\}_{(3)} & =-p . v \cdot \iint K\left(\xi^{\prime}-\xi\right) \times\left(\xi_{\alpha^{\prime}}^{\prime} \times \xi_{\beta^{\prime}}^{\prime}\right) \cdot \mathfrak{u}^{\prime} d \alpha^{\prime} d \beta^{\prime} e_{1} e_{2} e_{3} \\
& =-2 p \cdot v \cdot \iint\left(\left(\xi_{\alpha^{\prime}}^{\prime} \times \xi_{\beta^{\prime}}^{\prime}\right) \times \nabla_{\xi^{\prime}}\right) \Gamma\left(\xi^{\prime}-\xi\right) \cdot \mathfrak{u}^{\prime} d \alpha^{\prime} d \beta^{\prime} e_{1} e_{2} e_{3} \\
& =-2 p . v \cdot \iint\left(\xi_{\beta^{\prime}}^{\prime} \partial_{\alpha^{\prime}}-\xi_{\alpha^{\prime}}^{\prime} \partial_{\beta^{\prime}}\right) \Gamma\left(\xi^{\prime}-\xi\right) \cdot \mathfrak{u}^{\prime} d \alpha^{\prime} d \beta^{\prime} e_{1} e_{2} e_{3} \\
& =2 p . v \cdot \iint \Gamma\left(\xi^{\prime}-\xi\right)\left(\xi_{\beta^{\prime}}^{\prime} \cdot \partial_{\alpha^{\prime}} \mathfrak{u}^{\prime}-\xi_{\alpha^{\prime}}^{\prime} \cdot \partial_{\beta^{\prime}} \mathfrak{u}^{\prime}\right) d \alpha^{\prime} d \beta^{\prime} e_{1} e_{2} e_{3}
\end{aligned}
$$

therefore

$$
\begin{aligned}
\left\{\left(H_{\Sigma(t)} \mathfrak{u}\right)_{t t}\right\}_{(3)} & =2 p . v \cdot \iint \partial_{t}^{2}\left\{\Gamma\left(\xi^{\prime}-\xi\right)\right\}\left(\xi_{\beta^{\prime}}^{\prime} \cdot \partial_{\alpha^{\prime} \mathfrak{u}^{\prime}}-\xi_{\alpha^{\prime}}^{\prime} \cdot \partial_{\beta^{\prime}} \mathfrak{u}^{\prime}\right) d \alpha^{\prime} d \beta^{\prime} e_{1} e_{2} e_{3} \\
& +4 p . v \cdot \iint \partial_{t}\left\{\Gamma\left(\xi^{\prime}-\xi\right)\right\}\left(\xi_{\beta^{\prime}}^{\prime} \cdot \partial_{\alpha^{\prime}} \mathfrak{u}^{\prime}-\xi_{\alpha^{\prime}}^{\prime} \cdot \partial_{\beta^{\prime}} \mathfrak{u}^{\prime}\right)_{t} d \alpha^{\prime} d \beta^{\prime} e_{1} e_{2} e_{3} \\
& +2 p . v \cdot \iint \Gamma\left(\xi^{\prime}-\xi\right)\left(\xi_{\beta^{\prime}}^{\prime} \cdot \partial_{\alpha^{\prime}} \mathfrak{u}^{\prime}-\xi_{\alpha^{\prime}}^{\prime} \cdot \partial_{\beta^{\prime}} \mathfrak{u}^{\prime}\right)_{t t} d \alpha^{\prime} d \beta^{\prime} e_{1} e_{2} e_{3} .
\end{aligned}
$$

Since $N \mathcal{D}_{\xi}\left(\mathfrak{u}+H_{\Sigma(t)} \mathfrak{u}\right)=0$, we have the 3 -vector parts $N \times \mathcal{D}_{\xi} \cdot\left(\mathfrak{u}+\widetilde{H}_{\Sigma(t)} \mathfrak{u}\right) e_{1} e_{2} e_{3}=$ $-\left(N \cdot \mathcal{D}_{\xi}\right)\left\{H_{\Sigma(t)} \mathfrak{u}\right\}_{(3)}$. Therefore

$$
\begin{aligned}
& \left(\xi_{\beta} \cdot \partial_{\alpha} \mathfrak{u}-\xi_{\alpha} \cdot \partial_{\beta} \mathfrak{u}\right) e_{1} e_{2} e_{3}=\left(N \times \mathcal{D}_{\xi}\right) \cdot \mathfrak{u} e_{1} e_{2} e_{3} \\
& \quad=\frac{1}{2}\left(N \times \mathcal{D}_{\xi}\right) \cdot\left(\mathfrak{u}-\widetilde{H}_{\Sigma(t)} \mathfrak{u}\right) e_{1} e_{2} e_{3}-\frac{1}{2}\left(N \cdot \mathcal{D}_{\xi}\right)\left\{H_{\Sigma(t)} \mathfrak{u}\right\}_{(3)},
\end{aligned}
$$

and the term

$$
p . v . \iint \partial_{t}^{2}\left\{\Gamma\left(\xi^{\prime}-\xi\right)\right\}\left(\xi_{\beta^{\prime}}^{\prime} \cdot \partial_{\alpha^{\prime}} \mathfrak{u}^{\prime}-\xi_{\alpha^{\prime}}^{\prime} \cdot \partial_{\beta^{\prime}} \mathfrak{u}^{\prime}\right) d \alpha^{\prime} d \beta^{\prime} e_{1} e_{2} e_{3}=\mathcal{T}\left(\mathfrak{u}-H_{\Sigma(t)} \mathfrak{u}\right)
$$

where $\mathcal{T}$ is a bounded operator from $L_{2}$ to $L_{2}$.

We have that

$$
\begin{aligned}
\left(\xi_{\beta} \cdot \partial_{\alpha} \mathfrak{u}-\xi_{\alpha} \cdot \partial_{\beta} \mathfrak{u}\right)_{t} & =\xi_{\beta} \cdot \partial_{\alpha} \mathfrak{u}_{t}-\xi_{\alpha} \cdot \partial_{\beta} \mathfrak{u}_{t} \\
& =\xi_{\beta} \cdot \partial_{\alpha}\{\mathfrak{a}(\tilde{\mathbf{n}}-\mathbf{n})\}-\xi_{\alpha} \cdot \partial_{\beta}\{\mathfrak{a}(\tilde{\mathbf{n}}-\mathbf{n})\}
\end{aligned}
$$

here we used the fact that $\mathfrak{u}_{t}+e_{3}=\mathfrak{a} \tilde{\mathbf{n}}$ and

$$
\xi_{\beta} \cdot(a \mathbf{n})_{\alpha}-\xi_{\alpha} \cdot(a \mathbf{n})_{\beta}=0
$$


for any scalar-valued function $a$. Therefore

$$
\begin{aligned}
& \text { p.v. } \iint \partial_{t}\left\{\Gamma\left(\xi^{\prime}-\xi\right)\right\}\left(\xi_{\beta^{\prime}}^{\prime} \cdot \partial_{\alpha^{\prime}} \mathfrak{u}^{\prime}-\xi_{\alpha^{\prime}}^{\prime} \cdot \partial_{\beta^{\prime}} \mathfrak{u}^{\prime}\right)_{t} d \alpha^{\prime} d \beta^{\prime} e_{1} e_{2} e_{3} \\
& =p . v \cdot \iint \partial_{t}\left\{\Gamma\left(\xi^{\prime}-\xi\right)\right\}\left(\xi_{\beta^{\prime}}^{\prime} \cdot \partial_{\alpha^{\prime}}\left\{\mathfrak{a}^{\prime}\left(\tilde{\mathbf{n}}^{\prime}-\mathbf{n}^{\prime}\right)\right\}-\xi_{\alpha^{\prime}}^{\prime} \cdot \partial_{\beta^{\prime}}\left\{\mathfrak{a}^{\prime}\left(\tilde{\mathbf{n}}^{\prime}-\mathbf{n}^{\prime}\right)\right\}\right) d \alpha^{\prime} d \beta^{\prime} e_{1} e_{2} e_{3} \\
& =\mathcal{T}(\tilde{\mathbf{n}}-\mathbf{n}),
\end{aligned}
$$

where $\mathcal{T}$ is a bounded operator from $L_{2}$ to $L_{2}$.

Now we look at the last term in (7.8). From (7.9) and (7.10) we have

$$
\begin{aligned}
\left(\xi_{\beta} \cdot \partial_{\alpha} \mathfrak{u}-\xi_{\alpha} \cdot \partial_{\beta} \mathfrak{u}\right)_{t t} & =\xi_{t \beta} \cdot \partial_{\alpha}\{\mathfrak{a}(\tilde{\mathbf{n}}-\mathbf{n})\}-\xi_{t \alpha} \cdot \partial_{\beta}\{\mathfrak{a}(\tilde{\mathbf{n}}-\mathbf{n})\} \\
& +\xi_{\beta} \cdot \partial_{\alpha}\left\{\mathfrak{u}_{t t}-\mathfrak{a} \mathbf{n}_{t}\right\}-\xi_{\alpha} \cdot \partial_{\beta}\left\{\mathfrak{u}_{t t}-\mathfrak{a} \mathbf{n}_{t}\right\}
\end{aligned}
$$

Using (7.10), the fact that $\mathfrak{u}$ satisfies equation (5.21), Claim 5 and Claim 4, we get

$$
\begin{aligned}
& 2 \text { p.v. } \iint \Gamma\left(\xi^{\prime}-\xi\right)\left(\xi_{\beta^{\prime}}^{\prime} \cdot \partial_{\alpha^{\prime}} \mathfrak{u}^{\prime}-\xi_{\alpha^{\prime}}^{\prime} \cdot \partial_{\beta^{\prime}} \mathfrak{u}^{\prime}\right)_{t t} d \alpha^{\prime} d \beta^{\prime} e_{1} e_{2} e_{3} \\
& =2 p . v \cdot \iint \Gamma\left(\xi^{\prime}-\xi\right)\left(-\xi_{\beta^{\prime}}^{\prime} \cdot \partial_{\alpha^{\prime}}\left\{\mathfrak{a}^{\prime} \nabla_{\left.\mathbf{n}^{\prime} \mathfrak{u}^{\prime}+\mathfrak{a}^{\prime} \mathbf{n}_{t}^{\prime}\right\}}\right.\right. \\
& \left.\quad+\xi_{\alpha^{\prime}}^{\prime} \cdot \partial_{\beta^{\prime}}\left\{\mathfrak{a}^{\prime} \nabla_{\mathbf{n}^{\prime}} \mathfrak{u}^{\prime}+\mathfrak{a}^{\prime} \mathbf{n}_{t}^{\prime}\right\}\right) d \alpha^{\prime} d \beta^{\prime} e_{1} e_{2} e_{3}+\mathcal{T}_{1}(\tilde{\mathbf{n}}-\mathbf{n}) \\
& =-\left\{H_{\Sigma(t)}\left(\mathfrak{a} \nabla_{\mathbf{n}} \mathfrak{u}+\mathfrak{a n}_{t}\right)\right\}_{(3)}+\mathcal{T}_{1}(\tilde{\mathbf{n}}-\mathbf{n}) \\
& =-\mathfrak{a}\left\{H_{\Sigma(t)}\left(\nabla_{\mathbf{n}} \mathfrak{u}+\mathbf{n}_{t}\right)\right\}_{(3)}+\left\{\left[\mathfrak{a}, H_{\Sigma(t)}\right]\left(\nabla_{\mathbf{n}} \mathfrak{u}+\frac{N_{t}}{|N|}\right)\right\}_{(3)}+\mathcal{T}_{1}(\tilde{\mathbf{n}}-\mathbf{n}) \\
& =-\mathfrak{a}\left\{\nabla_{\mathbf{n}}\left(H_{\Sigma(t)} \mathfrak{u}\right)\right\}_{(3)}+\mathcal{T}_{1}(\tilde{\mathbf{n}}-\mathbf{n})+\mathcal{T}_{2}\left(\mathfrak{u}-H_{\Sigma(t)} \mathfrak{u}\right),
\end{aligned}
$$

where $\mathcal{T}_{1}$ and $\mathcal{T}_{2}$ are bounded operators from $L_{2}$ to $L_{2}$. This proves Claim 6 .

Now let's introduce a new vector $\hat{\mathbf{n}}$. We define $\hat{\mathbf{n}}=\frac{\left(\widetilde{H}_{\Sigma(t)} \mathfrak{u}\right)_{t}+e_{3}}{\left|\left(\widetilde{H}_{\Sigma(t)} \mathfrak{u}\right)_{t}+e_{3}\right|}$ to be the unit vector in the direction of $\left(\widetilde{H}_{\Sigma(t)} \mathfrak{u}\right)_{t}+e_{3}$. Then $\hat{\mathbf{n}}(0)=\tilde{\mathbf{n}}(0)$, and there is a time $0<T_{0} \leq T, T_{0}=T_{0}\left(C_{0}, c_{0}, \mu, M_{0}, M_{0,1}, \mathcal{M}_{0}\right)$, such that the vector $\hat{\mathbf{n}}$ is defined for $0 \leq t \leq T_{0}$. We know that for $0 \leq t \leq T_{0}, \tilde{\mathbf{n}}-\hat{\mathbf{n}}=\mathcal{T}\left(\left(\mathfrak{u}-H_{\Sigma(t)} \mathfrak{u}\right)_{t}\right)$, where $\mathcal{T}$ is a bounded operator from $L_{2}$ to $L_{2}$.

We are now ready to show that $\tilde{\mathbf{n}}=\mathbf{n}$ and $\mathfrak{u}=H_{\Sigma(t)} \mathfrak{u}$ for $0 \leq t \leq T$. Our idea is to show that $\mathfrak{u}-H_{\Sigma(t)} \mathfrak{u}$ and $\hat{\mathbf{n}}-\mathbf{n}$ satisfies a linear differential system, which together with the initial data $\hat{\mathbf{n}}(0)-\mathbf{n}(0)=0$ and $\mathfrak{u}_{0}-H_{\Sigma(t)} \mathfrak{u}_{0}=0$ implies that $\hat{\mathbf{n}}=\mathbf{n}$ and $\mathfrak{u}=H_{\Sigma(t)} \mathfrak{u}$ for $0 \leq t \leq T_{0}$. This would give that $\tilde{\mathbf{n}}=\hat{\mathbf{n}}=\mathbf{n}$ for $0 \leq t \leq T_{0}$. The same process can then be applied to $T_{0} \leq t \leq 2 T_{0}$, and we would have $\tilde{\mathbf{n}}=\mathbf{n}$ and $\mathfrak{u}=H_{\Sigma(t)} \mathfrak{u}$ for $T_{0} \leq t \leq 2 T_{0}$. After finitely many steps, we would have that $\tilde{\mathbf{n}}=\mathbf{n}$ and $\mathfrak{u}=H_{\Sigma(t)} \mathfrak{u}$ for all $0 \leq t \leq T$.

Let $\mathfrak{u}$ be a solution of (5.21)-(5.23), (5.29). It is easy to see from the derivations in $\S 5$ that $\mathfrak{u}$ satisfies

$$
\Re\left\{\tilde{\mathbf{n}}\left(I-H_{\Sigma(t)}\right)\left(\mathfrak{u}_{t t}+\mathfrak{a} \nabla_{\mathbf{n}} \mathfrak{u}-\mathfrak{U}_{t t}-\mathfrak{a}(\tilde{\mathbf{n}} \cdot \nabla) \mathfrak{U}\right)\right\}=0,
$$

therefore

$$
\Re\left\{\mathbf{n}\left(I-H_{\Sigma(t)}\right)\left(\mathfrak{u}_{t t}+\mathfrak{a} \nabla_{\mathbf{n}} \mathfrak{u}-\mathfrak{U}_{t t}-\mathfrak{a}(\mathbf{n} \cdot \nabla) \mathfrak{U}\right)\right\}=\mathcal{T}(\tilde{\mathbf{n}}-\mathbf{n}),
$$


where $\mathcal{T}$ is a bounded linear operator from $L_{2}$ to $L_{2}$. Now

$$
\begin{aligned}
(I- & \left.H_{\Sigma(t)}\right)\left(\mathfrak{u}_{t t}+\mathfrak{a} \nabla_{\mathbf{n}} \mathfrak{u}-\mathfrak{U}_{t t}-\mathfrak{a} \nabla_{\mathbf{n}} \mathfrak{U}\right) \\
& =2\left(\mathfrak{u}_{t t}+\mathfrak{a} \nabla_{\mathbf{n}} \mathfrak{u}-\mathfrak{U}_{t t}-\mathfrak{a} \nabla_{\mathbf{n}} \mathfrak{U}\right)-\left(I+H_{\Sigma(t)}\right)\left(\mathfrak{u}_{t t}+\mathfrak{a} \nabla_{\mathbf{n}} \mathfrak{u}-\mathfrak{U}_{t t}-\mathfrak{a} \nabla_{\mathbf{n}} \mathfrak{U}\right) \\
& =\left(\mathfrak{u}-H_{\Sigma(t)} \mathfrak{u}\right)_{t t}+\mathfrak{a} \nabla_{\mathbf{n}}\left(\mathfrak{u}-H_{\Sigma(t)} \mathfrak{u}\right)+\frac{1}{2}\left[\partial_{t}^{2}, H_{\Sigma(t)}\right]\left(\mathfrak{u}-H_{\Sigma(t)} \mathfrak{u}\right) \\
& -\frac{1}{2}\left(I+H_{\Sigma(t)}\right)\left(\mathfrak{a}\left(\nabla_{\mathbf{n}}+\nabla_{\mathbf{n}}^{-}\right)\left(\mathfrak{u}-H_{\Sigma(t)} \mathfrak{u}\right)\right) \\
& -\left[\mathfrak{a} n_{1}, H\right] \partial_{x}^{-}\left(\mathfrak{u}-H_{\Sigma(t)} \mathfrak{u}\right)-\left[\mathfrak{a} n_{2}, H\right] \partial_{y}^{-}\left(\mathfrak{u}-H_{\Sigma(t)} \mathfrak{u}\right) \\
& -\left[\mathfrak{a} n_{3}, H\right] \partial_{z}^{-}\left(\mathfrak{u}-H_{\Sigma(t)} \mathfrak{u}\right),
\end{aligned}
$$

where $n_{i}, i=1,2,3$, are components of $\mathbf{n}$, so

$$
\begin{aligned}
\left(I-H_{\Sigma(t)}\right)\left(\mathfrak{u}_{t t}+\mathfrak{a} \nabla_{\mathbf{n}} \mathfrak{u}-\mathfrak{U}_{t t}-\mathfrak{a} \nabla_{\mathbf{n}} \mathfrak{U}\right) & =\left(\mathfrak{u}-H_{\Sigma(t)} \mathfrak{u}\right)_{t t}+\mathfrak{a} \nabla_{\mathbf{n}}\left(\mathfrak{u}-H_{\Sigma(t)} \mathfrak{u}\right) \\
& +\mathcal{T}_{1}\left(\mathfrak{u}-H_{\Sigma(t)} \mathfrak{u}\right)+\mathcal{T}_{2}\left(\left(\mathfrak{u}-H_{\Sigma(t)} \mathfrak{u}\right)_{t}\right),
\end{aligned}
$$

where $\mathcal{T}_{1}, \mathcal{T}_{2}$ are bounded operators from $L_{2}$ to $L_{2}$. Therefore from Claim 6 ,

$$
\begin{aligned}
\{(I & \left.\left.-H_{\Sigma(t)}\right)\left(\mathfrak{u}_{t t}+\mathfrak{a} \nabla_{\mathbf{n}} \mathfrak{u}-\mathfrak{U}_{t t}-\mathfrak{a} \nabla_{\mathbf{n}} \mathfrak{U}\right)\right\}_{(3)} \\
& =-\left\{\left(H_{\Sigma(t)} \mathfrak{u}\right)_{t t}+\mathfrak{a} \nabla_{\mathbf{n}}\left(H_{\Sigma(t)} \mathfrak{u}\right)\right\}_{(3)}+\mathcal{T}_{1}\left(\mathfrak{u}-H_{\Sigma(t)} \mathfrak{u}\right)+\mathcal{T}_{2}\left(\left(\mathfrak{u}-H_{\Sigma(t)} \mathfrak{u}\right)_{t}\right) \\
& =\mathcal{T}_{1}\left(\mathfrak{u}-H_{\Sigma(t)} \mathfrak{u}\right)+\mathcal{T}_{2}\left(\left(\mathfrak{u}-H_{\Sigma(t)} \mathfrak{u}\right)_{t}\right)+\mathcal{T}_{3}(\hat{\mathbf{n}}-\mathbf{n}),
\end{aligned}
$$

where $\mathcal{T}_{1}, \mathcal{T}_{2} \mathcal{T}_{3}$ are bounded operators from $L_{2}$ to $L_{2}$. Now

$$
\begin{aligned}
F & =\left(I-H_{\Sigma(t)}\right)\left(\mathfrak{u}_{t t}+\mathfrak{a} \nabla_{\mathbf{n}} \mathfrak{u}-\mathfrak{U}_{t t}-\mathfrak{a} \nabla_{\mathbf{n}} \mathfrak{U}\right) \\
& -\left(I-H_{\Sigma(t)}\right)(I-\mathcal{K})^{-1}\left\{\left(I-H_{\Sigma(t)}\right)\left(\mathfrak{u}_{t t}+\mathfrak{a} \nabla_{\mathbf{n}} \mathfrak{u}-\mathfrak{U}_{t t}-\mathfrak{a} \nabla_{\mathbf{n}} \mathfrak{U}\right)\right\}_{(3)}
\end{aligned}
$$

is a 1-vector-valued function satisfying the assumption of Claim 2. From (7.2), (7.11) and (7.13), we get

$$
F=\mathcal{T}_{1}\left(\mathfrak{u}-H_{\Sigma(t)} \mathfrak{u}\right)+\mathcal{T}_{2}\left(\left(\mathfrak{u}-H_{\Sigma(t)} \mathfrak{u}\right)_{t}\right)+\mathcal{T}_{3}(\hat{\mathbf{n}}-\mathbf{n}),
$$

where $\mathcal{T}_{1}, \mathcal{T}_{2} \mathcal{T}_{3}$ are bounded from $L_{2}$ to $L_{2}$. Consequently from (7.12) and (7.13),

$$
\begin{aligned}
& \left(\mathfrak{u}-H_{\Sigma(t)} \mathfrak{u}\right)_{t t}+\mathfrak{a} \nabla_{\mathbf{n}}\left(\mathfrak{u}-H_{\Sigma(t)} \mathfrak{u}\right) \\
& \quad=\mathcal{T}_{1}\left(\mathfrak{u}-H_{\Sigma(t)} \mathfrak{u}\right)+\mathcal{T}_{2}\left(\left(\mathfrak{u}-H_{\Sigma(t)} \mathfrak{u}\right)_{t}\right)+\mathcal{T}_{3}(\hat{\mathbf{n}}-\mathbf{n}),
\end{aligned}
$$

where $\mathcal{T}_{1}, \mathcal{T}_{2} \mathcal{T}_{3}$ are operators bounded from $L_{2}$ to $L_{2}$.

Now we want to derive an equation of $(\hat{\mathbf{n}}-\mathbf{n})$. Let

$$
\mathfrak{c}=-\left(I+\widetilde{\mathcal{K}^{*}}\right)^{-1}\left(\Re\left\{\tilde{\mathbf{n}} f\left(\xi, \mathfrak{u}, \mathfrak{u}_{t}\right)\right\}\right) .
$$

(5.21) becomes $\mathfrak{u}_{t t}+\mathfrak{a} \nabla_{\mathbf{n}} \mathfrak{u}=\tilde{\mathbf{n}} \mathfrak{c}$. Let $\mathfrak{b}=\left|\left(\widetilde{H}_{\Sigma(t)} \mathfrak{u}\right)_{t}+e_{3}\right|$. Then $\left(\widetilde{H}_{\Sigma(t)} \mathfrak{u}\right)_{t}+e_{3}=\mathfrak{b} \hat{\mathbf{n}}$ and

$$
\left(\widetilde{H}_{\Sigma(t)} \mathfrak{u}\right)_{t t}=\mathfrak{b} \hat{\mathbf{n}}_{t}+\mathfrak{b}_{t} \hat{\mathbf{n}} .
$$

From (7.14), we have

$$
\begin{aligned}
& \left(H_{\Sigma(t)} \mathfrak{u}\right)_{t t}+\mathfrak{a} \nabla_{\mathbf{n}}\left(H_{\Sigma(t)} \mathfrak{u}\right) \\
& \quad=\tilde{\mathbf{n}} \mathfrak{c}+\mathcal{T}_{1}\left(\mathfrak{u}-H_{\Sigma(t)} \mathfrak{u}\right)+\mathcal{T}_{2}\left(\left(\mathfrak{u}-H_{\Sigma(t)} \mathfrak{u}\right)_{t}\right)+\mathcal{T}_{3}(\hat{\mathbf{n}}-\mathbf{n}) \\
& \quad=\hat{\mathbf{n}} \mathfrak{c}+\mathcal{T}_{1}\left(\mathfrak{u}-H_{\Sigma(t)} \mathfrak{u}\right)+\mathcal{T}_{2}\left(\left(\mathfrak{u}-H_{\Sigma(t)} \mathfrak{u}\right)_{t}\right)+\mathcal{T}_{3}(\hat{\mathbf{n}}-\mathbf{n}),
\end{aligned}
$$


therefore

$$
\begin{aligned}
\mathfrak{b} \hat{\mathbf{n}}_{t} & =-\mathfrak{a} \nabla_{\mathbf{n}}\left(\widetilde{H}_{\Sigma(t)} \mathfrak{u}\right)+\hat{\mathbf{n}} \mathfrak{c}-\mathfrak{b}_{t} \hat{\mathbf{n}}+\mathcal{T}_{1}\left(\mathfrak{u}-H_{\Sigma(t)} \mathfrak{u}\right)+\mathcal{T}_{2}\left(\left(\mathfrak{u}-H_{\Sigma(t)} \mathfrak{u}\right)_{t}\right)+\mathcal{T}_{3}(\hat{\mathbf{n}}-\mathbf{n}) \\
& =\left(\mathfrak{a} \nabla_{\mathbf{n}}\left(\widetilde{H}_{\Sigma(t)} \mathfrak{u}\right) \times \hat{\mathbf{n}}_{t}\right) \times \hat{\mathbf{n}}_{t}+\mathcal{T}_{1}\left(\mathfrak{u}-H_{\Sigma(t)} \mathfrak{u}\right)+\mathcal{T}_{2}\left(\left(\mathfrak{u}-H_{\Sigma(t)} \mathfrak{u}\right)_{t}\right)+\mathcal{T}_{3}(\hat{\mathbf{n}}-\mathbf{n}) .
\end{aligned}
$$

Since $\mathfrak{a}-\mathfrak{b}=\mathcal{T}\left(\left(\mathfrak{u}-H_{\Sigma(t)} \mathfrak{u}\right)_{t}\right)$ is a bounded operator from $L_{2}$ to $L_{2}$, we have

$$
\hat{\mathbf{n}}_{t}=\left(\nabla_{\mathbf{n}}\left(\widetilde{H}_{\Sigma(t)} \mathfrak{u}\right) \times \hat{\mathbf{n}}_{t}\right) \times \hat{\mathbf{n}}_{t}+\mathcal{T}_{1}\left(\mathfrak{u}-H_{\Sigma(t)} \mathfrak{u}\right)+\mathcal{T}_{2}\left(\left(\mathfrak{u}-H_{\Sigma(t)} \mathfrak{u}\right)_{t}\right)+\mathcal{T}_{3}(\hat{\mathbf{n}}-\mathbf{n}) .
$$

Combining with (7.4), we obtain

$$
(\hat{\mathbf{n}}-\mathbf{n})_{t}=\mathcal{T}_{1}\left(\mathfrak{u}-H_{\Sigma(t)} \mathfrak{u}\right)+\mathcal{T}_{2}\left(\left(\mathfrak{u}-H_{\Sigma(t)} \mathfrak{u}\right)_{t}\right)+\mathcal{T}_{3}(\hat{\mathbf{n}}-\mathbf{n}) .
$$

Using the results in the estimate part of $\S 6$, it is easy to establish an energy estimate for system (7.14),(7.17). With the initial data $\hat{\mathbf{n}}(0)=\mathbf{n}(0)$ and $\mathfrak{u}_{0}=$ $H_{\Sigma(0)} \mathfrak{u}_{0}$, we conclude that

$$
\hat{\mathbf{n}}(t)=\mathbf{n}(t) \quad \text { and } \quad \mathfrak{u}(t)=H_{\Sigma(t)} \mathfrak{u}(t), \quad \text { for } 0 \leq t \leq T_{0},
$$

so $\tilde{\mathbf{n}}(t)=\hat{\mathbf{n}}(t)=\mathbf{n}(t)$ for $0 \leq t \leq T_{0}$. Now taking $T_{0}$ as the initial time, we obtain

$$
\tilde{\mathbf{n}}(t)=\mathbf{n}(t) \quad \text { and } \quad \mathfrak{u}(t)=H_{\Sigma(t)} \mathfrak{u}(t), \quad \text { for } T_{0} \leq t \leq 2 T_{0} .
$$

After finitely many steps, we get

$$
\tilde{\mathbf{n}}(t)=\mathbf{n}(t) \quad \text { and } \quad \mathfrak{u}(t)=H_{\Sigma(t)} \mathfrak{u}(t), \quad \text { for } 0 \leq t \leq T .
$$

This proves that a solution of (5.21)-(5.23), (5.29) is also a solution of (4.3),(4.4), $\left(4.5^{\prime}\right)$.

We can now state the well-posedness result of the nonlinear waterwave system $(4.3),(4.4),\left(4.5^{\prime}\right)$.

Let $\xi_{0}, \xi_{1}=\mathfrak{u}_{0}$ be as given in the initial data part of $\S 5$. In particular, let $\Sigma(0): \xi=\xi_{0}(\alpha, \beta)$ be a surface that divides $R^{3}$ into two simply connected regions. Assume that there are vectors $e_{\alpha}, e_{\beta}$, such that $e_{\alpha} \times e_{\beta}=e_{3}, \xi_{0, \alpha}-e_{\alpha}, \xi_{0, \beta}-e_{\beta} \in$ $H^{s-1 / 2}\left(R^{2}, \mathcal{C}\left(V_{3}\right)\right)$; and there are constants $\mu>0, C_{0}>0$, such that

$$
\begin{aligned}
& \left|\xi_{0, \alpha} \times \xi_{0, \beta}\right| \geq 2 \mu, \quad \text { for }-\infty<\alpha, \beta<\infty, \\
& \left|\xi_{0}(\alpha, \beta)-\xi_{0}\left(\alpha^{\prime}, \beta^{\prime}\right)\right| \geq 2 C_{0}\left(\left|\alpha-\alpha^{\prime}\right|+\left|\beta-\beta^{\prime}\right|\right), \quad \text { for }-\infty<\alpha, \beta<\infty .
\end{aligned}
$$

Assume that $\mathbf{n}_{0}-e_{3} \in H^{s}$, where $\mathbf{n}_{0}=\frac{\xi_{0, \alpha} \times \xi_{0, \beta}}{\left|\xi_{0, \alpha} \times \xi_{0, \beta}\right|}$.

Theorem 7.1. Let $s \geq 5 / 2+q$, where $q>1$. Assume further that

$$
\xi_{1} \in H^{s+1 / 2}\left(R^{2}, \mathcal{C}\left(V_{3}\right)\right) .
$$

Then there exists $T>0$, such that the system (4.3),(4.4),(4.5') has a unique solution $\xi=\xi(\alpha, \beta, t)$ for $0 \leq t \leq T$, with the property that the surfaces $\Sigma(t): \xi=$ $\xi(\alpha, \beta, t), 0 \leq t \leq T$, satisfy $H 1-H 3$ of $\S 6$, and

$$
\xi_{t} \in C^{j}\left([0, T], H^{s+1 / 2-j / 2}\left(R^{2}, \mathcal{C}\left(V_{3}\right)\right)\right),
$$

for $j=0,1,2$. 


\section{ACKNOWLEDGMENT}

I would like to thank Russ Caflisch and Tom Hou for their idea about the sign condition and for my very pleasant visit at UCLA and Caltech; I would also like to thank Tom Beale for referring me to the work of Andreev [26]. I have talked about the results in this paper in seminars at UCLA, Brown University, IAS, Courant Institute, Princeton University, University of Arizona, the AMS meeting at College Park, Maryland, and the workshop at MSRI; comments and questions from audiences have influenced the final outcome of this paper. I would like to thank my hosts and audiences in my talks. In particular, I would like to thank Walter Craig, Carlos Kenig and Lihe Wang. I would also like to thank Siye Wu for discussions on the physical meaning of this and my previous work.

\section{REFERENCES}

1. T. Beale, T. Hou and J. Lowengrub, Growth rates for the linearized motion of fluid interfaces away from equilibrium, Comm. Pure Appl. Math. V.46 no.9, 1993, 1269-1301. MR 95c:76016

2. F. Brackx, R. Delanghe and F. Sommen, Clifford Analysis, Research Notes in Math. Pitman, 1982. MR 85j:30103

3. R. E. Caflisch, Mathematical Analysis of Vortex Dynamics, Mathematical Aspects of Vortex Dynamics, edited by R. E. Caflisch, Philadelphia : Society for Industrial and Applied Mathematics 1988. CMP 21:14

4. R. Coifman, A. McIntosh and Y. Meyer, L'intégrale de Cauchy definit un operateur borne sur $L^{2}$ pour les courbes lipschitziennes, Ann. of Math. (2), 116 (1982), 361-387. MR 84m:42027

5. R. Coifman, G. David and Y. Meyer, La solution des conjectures de Calderón, Adv. in Math. 48, 1983, pp.144-148. MR 84i:42025

6. W. Craig, An existence theory for water waves and the Boussinesq and Korteweg-deVries scaling limits, Comm. in P. D. E. 10(8), 1985, pp.787-1003. MR 87f:35210

7. G. B. Folland, Introduction to Partial Differential Equations, Princeton Univ. Press, 1976. MR 58:29031

8. G. Birkhoff, Helmholtz and Taylor instability, Proc. Symp. in Appl. Math. Vol. XIII, pp. 55-76. MR 25:875

9. D. Gilbarg and N. S. Trudinger, Elliptic Partial Differential Equations of Second Order. MR 86c:35035

10. J. Gilbert and M. Murray, Clifford Algebras and Dirac Operators in Harmonic Analysis, Cambridge University Press, 1991. MR 93e:42027

11. T. Hou, Z. Teng and P. Zhang, Well-posedness of linearized motion for 3-D water waves far from equilibrium, Comm. in PDE, Vol.21, 1996, pp. 1551-1585. MR 98c:76013

12. T. Kano and T. Nishida, Sur les ondes de surface de l'eau avec une justification mathématique des equations des ondes en eau peu profonde, J. Math. Kyoto Univ. 19(2), 1979, pp 335-370. MR 82d:76012

13. C. Kenig, Elliptic boundary value problems on Lipschitz domains, Beijing Lectures in Harmonic Analysis, ed. by E. M. Stein, Princeton Univ. Press, 1986, pp. 131-183. MR 88a:35066

14. C. Kenig, Harmonic analysis techniques for second order Elliptic boundary value problems, CBMS no. 83, AMS, 1994. MR 96a:35040

15. M. Mitrea, Clifford wavelets, singular integrals, and Hardy spaces, Lecture notes in math. 1575, Springer 1994. MR 96e:31005

16. S. Mizohata, The theory of partial differential equations, Cambridge Univ. Press, 1973. MR 58:29033

17. M. Murrey, The Cauchy intergal, Calderón commutators, and conjugations of singular integrals in $R^{n}$, Trans. Amer. Math. Soc. 289 (1985), no.2, 497-518. MR 86f:42009

18. V. I. Nalimov, The Cauchy-Poisson Problem (in Russian), Dynamika Splosh. Sredy 18, 1974, pp. 104-210. MR 58:29458

19. M. Shinbrot, The initial value problem for surface waves under gravity, I. The simplest case, Indiana U. Math. J. 25, 1976, pp 281-300. MR 53:7211

20. E. M. Stein and G. Weiss, Introduction to Fourier Analysis on Euclidean Spaces, Princeton University Press, N.J., 1971. MR 46:4102 
21. J. J. Stoker, Water Waves, Interscience Publishers, New York, 1957. MR 21:2438

22. G. Taylor, The instability of liquid surfaces when accelerated in a direction perpendicular to their planes I., Proc. Roy. Soc. London A 201, 1950, 192-196. MR 12:58f

23. G. C. Verchota, Layer potentials and boundary value problems for Laplace's equation in Lipschitz domains, Thesis, University of Minnesota, 1982, J. of Func. Analysis, 59 (1984), 572-611. MR 86e: 35038

24. S. Wu, Well-posedness in Sobolev spaces of the full water wave problem in 2-D, Invent. Math., 130 (1997), pp.39-72. MR 98m:35167

25. H. Yosihara, Gravity waves on the free surface of an incompressible perfect fluid of finite depth, RIMS Kyoto 18, 1982, pp. 49-96. MR 83k:76017

26. V. K. Andreev, Stability of Unsteady Motions of a Fluid with a Free Boundary, VO "Nauka", Novosibirsk, 1992 (in Russian). MR 94j:76001

Department of Mathematics, The University of Iowa, Iowa City, Iowa 52242

E-mail address: swu@math.uiowa.edu

Current address: Department of Mathematics, University of Maryland, College Park, Maryland 20742

E-mail address: sijue@math.umd.edu 